\title{
A local well-posedness result for the quasilinear wave equation in $\mathbb{R}^{2+1}$
}

\author{
Dan-Andrei Geba \\ Department of Mathematics, Evans Hall \\ University of California at Berkeley, Berkeley, CA 94720-3840
}

\begin{abstract}
We investigate the local well-posedness for quasilinear wave equations in $\mathbb{R}^{2+1}$. Our work extends the geometric methods pioneered by Klainerman and KlainermanRodnianski for similar problems in $\mathbb{R}^{3+1}$. The main new ingredient of the argument is the use of two new vectorfields, the scaling vectorfield $S$ and the angular momentum vectorfield $\Omega$, which complement the decay information provided by the Morawetz vectorfield $K$.
\end{abstract}

\section{Introduction}

In this article we consider the following Cauchy problem:

$$
\begin{aligned}
& \partial_{t}^{2} \phi-g^{i j}(\phi) \partial_{i} \partial_{j} \phi=N(\phi, \partial \phi), \\
& \phi(0, x)=\phi_{0}(x), \partial_{t} \phi(0, x)=\phi_{1}(x),(t, x) \in \mathbb{R}^{1+n} .
\end{aligned}
$$

where the metric $g(u)=\left(g_{i j}(u)\right)_{i, j=1, \ldots, n}$ is a smooth, uniformly positive definite matrix and the nonlinearity $N(\phi, \partial \phi)$ is quadratic in $\partial \phi$. Assuming that the initial data satisfies $\left(\phi_{0}, \phi_{1}\right) \in H^{s}\left(\mathbb{R}^{n}\right) \times H^{s-1}\left(\mathbb{R}^{n}\right)$, we will be interested in the local well-posedness of the initial value problem (1), e.g. for what values of $s$ there exists a unique local solution

$$
\phi \in C\left([0, T], H^{s}\left(\mathbb{R}^{n}\right)\right) \cap C^{1}\left([0, T], H^{s-1}\left(\mathbb{R}^{n}\right)\right) .
$$

The expected range for $s$ is

$$
s>\max \left\{\frac{n}{2}, \frac{n+5}{4}\right\}
$$

where the first exponent comes from scaling, while the second one, according to Linblad [9], is connected with the concentration of null rays. Recently, Smith and Tataru [15] proved 
that this is the range in the case of the dimensions $n=2,3$ for general systems of quasilinear wave equations, while Klainerman and Rodnianski [8] showed it in the particular case of the Einstein vacuum equations $(n=3)$. For $\phi$ a smooth solution of equation (1), we have the following $H^{s}$-energy estimate, true for any $s>0$ :

$$
\|\partial \phi(t)\|_{H^{s-1}} \lesssim\|\partial \phi(0)\|_{H^{s-1}} \cdot \exp \left(\int_{0}^{t}\|\partial \phi(\tau)\|_{L_{x}^{\infty}} d \tau\right)
$$

In order to obtain a local well-posedness result, one needs to control the integral term in (3). The 'classical' approach is by using the Sobolev inequality :

$$
\int_{0}^{t}\|\partial \phi(\tau)\|_{L_{x}^{\infty}} d \tau \leq t \cdot \sup _{0 \leq \tau \leq t}\|\partial \phi(\tau)\|_{H_{x}^{s-1}}
$$

where $s$ needs to satisfy $s>\frac{n}{2}+1$. Combining (3) and (4), together with a Picard iteration argument, we obtain local well-posedness for $s$ in the range $s>\frac{n}{2}+1$. In the form presented above, the result appears in a paper by Hughes-Kato-Marsden [3]. Another way of estimating the integral term $\int_{0}^{t}\|\partial \phi(\tau)\|_{L_{x}^{\infty}} d \tau$, locally in time, is through Strichartz estimates for $\|\partial \phi\|_{L_{t}^{4} L_{x}^{\infty}}(n=2)$ and $\|\partial \phi\|_{L_{t}^{2} L_{x}^{\infty}}(n \geq 3)$. Using such estimates, Ponce and Sideris [11] proved that the semilinear wave equation $\square \phi=|\partial \phi|^{2}$ is well-posed in $H^{\frac{n}{2}+\frac{3}{4}+\epsilon}\left(\mathbb{R}^{n}\right)$ for $n=2$ and $H^{\frac{n}{2}+\frac{1}{2}+\epsilon}\left(\mathbb{R}^{n}\right)$ when $n \geq 3$. The scheme to keep in mind when attempting to prove a local well-posedness result for the equation (1), based on Strichartz estimates (and of course energy estimates), is captured in the following:

Heuristic argument In view of the energy estimate (3), one can make the bootstrap assumptions $\phi \in C\left([0, T], H^{s}\left(\mathbb{R}^{n}\right)\right)$ and $\partial \phi \in L_{t}^{1} L_{x}^{\infty}$. This implies that the metric $g=g(\phi)$ satisfies the same conditions. So under these assumptions for $g$, one needs to prove Strichartz estimates for the wave operator $\square_{g}=\partial_{t}^{2}-g^{i j}(t, x) \partial_{i} \partial_{j}$, with $s \geq s_{0}$. This, in turn, will imply local well-posedness in the space $H^{s_{0}}$.

The first results were proved by Kapitanski [4] and Mockenhaupt-Seeger-Sooge [10] for smooth metrics. A significant improvement came later, when Smith [12] proved, for $n=2,3$, the full Strichartz estimates for metrics with $C^{2}$ coefficients. This is the sharp result, as it was shown later by a counterexample in Smith-Sogge [13]. Smith relied on a technique based on the approximation of the solution using a wave packet decomposition. This construction turned out to be very important and was also used in the recent paper of Smith and Tataru [15]. Observe that according to this scheme, in order to apply Smith's result, one needs $H^{s}$-regularity of the coefficients, with $s>\frac{n}{2}+2$, and so is not able to recover even the classical result corresponding to $s>\frac{n}{2}+1$. This is why one needs Strichartz-type estimates for metrics with very rough coefficients. An important breakthrough took place when it was first realized, independently by Bahouri-Chemin [1], [2] and Tataru [16], that in order to go below this $C^{2}$ assumption on the coefficients, one needs to allow losses in the Strichartz 
estimates which, though will not give the optimal expected result (2) for $n=2,3$, it will nevertheless improve the classical result $\left(s>\frac{n}{2}+1\right)$. More precisely, Bahouri-Chemin [1], [2] and Tataru [16] were able to show that for metrics with coefficients rougher than $C^{2}$ the following Strichartz estimates hold

$$
\begin{aligned}
\|\partial \varphi\|_{L_{t}^{2} L_{x}^{\infty}} \lesssim\|\varphi(0)\|_{H^{\frac{n}{2}+\frac{1}{2}+\frac{1}{4}}}+\|\varphi(0)\|_{H^{\frac{n}{2}-\frac{1}{2}+\frac{1}{4}}} & n \geq 3 \\
\|\partial \varphi\|_{L_{t}^{4} L_{x}^{\infty}} \lesssim\|\varphi(0)\|_{H^{\frac{n}{2}+\frac{3}{4}+\frac{1}{8}}}+\|\varphi(0)\|_{H^{\frac{n}{2}-\frac{1}{4}+\frac{1}{8}}} & n=2 .
\end{aligned}
$$

Both papers are based on a parametrix construction for the local linearized problem on small time intervals, followed by a summation of the estimates obtained on each one of these intervals. Bahouri and Chemin used Fourier integral operators, while Tataru's proof relied on the FBI transform to localize precisely in space and frequency. Using similar ideas, he further improved in [17] the local well-posedness theory for equation (1) up to $s>\frac{n}{2}+\frac{5}{6}$, when $n=2$, and $s>\frac{n}{2}+\frac{2}{3}$, for $n \geq 3$. A later paper by Smith and Tataru [14] showed that based on the heuristic argument previously presented, one cannot improve this result of Tataru. One needs to stress the idea that all these results were based on regularity assumptions on the coefficients of the metric, without taking advantage of the special structure of the nonlinear equation. An important next step was taken by Klainerman in [6] and by Klainerman and Rodnianski in [7], leading to further improvements in the local well-posedness theory. The main new observation is that, due to the quasilinear structure of the equation, the coefficients $g^{i j}(\phi)$ of the equation (1) verify themselves an equation of the type

$$
\square_{g} g^{i j}=N^{\prime}
$$

where $N^{\prime}$ depends only on $\phi$ and $\partial \phi$. The term $\square_{g} g$ appears crucially in the structure of the Raychaudhuri equation for one of the Ricci coefficients and so it allows us to prove that this Ricci coefficient is smoother than it was previously believed. Based on elliptic estimates, one can then show that all Ricci coefficients are smoother. Both papers, dealing with the case $n=3$, are based on a geometric approach, using the foliation of the spacetime by null cones and commutation of the linearized local equation with the modified version of Morawetz vectorfield in order to prove that the conformal energy associated with the linearized equation is bounded. The first paper by Klainerman [6] reproved Tataru's result [17], while the second one [7] by Klainerman and Rodnianski improved it up to $s>\frac{n}{2}+\frac{1}{2}+\frac{2-\sqrt{3}}{2}$ in the case when $n=3$. The recent papers who proved the sharp result, [8] and [15], used a variation of the same idea explained above, the former one combining it with a wave packet decomposition along the lines of [12]. Our work, which is in the spirit of Klainerman and Rodnianski program, deals with the case $n=2$. In this situation using only the Morawetz vectorfield does not longer suffice to derive the needed decay information as in the case of dimension $n=3$. Instead, and this is the main novelty of our argument, we rely on two 
other vectorfields, the scaling and the angular momentum vectorfield, which complement the information provided by the Morawetz vectorfield. Using this method we improve the previous result of Tataru [17], but we fail to match the sharp one $s>\frac{7}{4}$. Our main result is the following:

Theorem 1.1 The Cauchy problem (1) with the metric $g^{i j}$ satisfying the assumptions below, is locally well posed in $H^{s}$ for any $s>s_{0}=\frac{7}{4}+\frac{5-\sqrt{22}}{4}$. Moreover, $\phi$ satisfies the following Strichartz type estimate ${ }^{1}$

$$
\|\partial \phi\|_{L_{[0, T]}^{4} L_{x}^{\infty}} \leq c T^{s-s_{0}}\|\phi[0]\|_{H^{s}}
$$

Conditions satisfied by the coefficients $g^{i j}$ : The metric $g^{i j}=g^{i j}(z)$ is smooth and uniformly positive definite with respect to bounded values of the parameter $z \in \mathbb{R}$. Namely, there exist positive constants $M_{0}, A_{0}$ such that for a sufficiently large integer $k$

$$
\begin{aligned}
& \sup _{|z| \leq A_{0}}\left|\left(\frac{d}{d z}\right)^{l} g^{i j}\right| \leq M_{0}, \quad \forall 0 \leq l \leq k \\
& M_{0}^{-1}|\xi|^{2} \leq g^{i j}(z) \xi_{i} \xi_{j} \leq M_{0}|\xi|^{2}, \quad \forall|z| \leq A_{0}, \\
& N(\phi, \partial \phi)=\sum_{\alpha, \beta} N^{\alpha \beta}(\phi) \partial_{\alpha} \phi \partial_{\beta} \phi, \quad \sup _{|z| \leq A_{0}}\left|\left(\frac{d}{d z}\right)^{l} N^{\alpha \beta}(z)\right| \leq M_{0}, \quad \forall 0 \leq l \leq k .
\end{aligned}
$$

First we will reduce the problem to the proof of the boundedness of the conformal energy for the solution of the reduced linear equation. This reduction is by now classical, see [6], [7] and [8]. It is based on the parradiferential calculus in which one localizes the solution to a certain dyadic frequency $\lambda$ and the coefficients at frequency at most $\lambda^{a}$, for an $a \in(0,1)$. This is followed by a $T T^{*}$-type argument which reduces the Strichartz estimate to a dispersive inequality. Finally, using Sobolev and trace theorems specially adapted to our situation, we showed how to reduce our dispersive inequality to a bound for the conformal energy associated with the linearized local problem. Secondly we will turn our attention to the geometry of the null cones. Here the important tools are: the null frame $\{L, \underline{L}, A\}$, the optical function $u$, the lapse function $b$, the affine parameter $s$, the second fundamental form $\kappa$ and the Ricci coefficients $\chi, \underline{\chi}, \eta, \underline{\eta}, \underline{\xi}$. The main result is the proof of asymptotic properties for the Ricci coefficients and for the lapse function. The last and the most important part of the proof deals with the commutation between the wave operator $\square_{g}$ and the special vectorfields: the Morawetz vectorfield $K$, the scaling vectorfield $S$ and the angular momentum vectorfield $\Omega$. When commuting we obtain error terms which are expressed relative to the deformation tensor of these vectorfields. The deformation tensor is then estimated in terms

\footnotetext{
${ }^{1}$ We denote the initial data for the equation (1) by $\phi[0]$ and say that $\phi[0] \in H^{s}$ if $\left(\phi_{0}, \phi_{1}\right) \in H^{s} \times H^{s-1}$.
} 
of the asymptotics for the Ricci coefficients, deduced in the previous step. Boundedness for the conformal energy is finally obtained by careful integration of the error terms.

\section{The reduction of theorem 1.1 to a dispersive in- equality}

As mentioned in the introduction, this reduction is essentially the same with the one presented in [6], [7] and [8]. This is why we will only sketch its main steps and refer the reader to [7] for more details. We start first with:

\section{$2.1 \quad$ Bootstrap argument}

In order to prove Theorem 1.1 we have to obtain the following inequality ${ }^{2}$

$$
\|\partial \phi\|_{L_{[0, T]}^{1} L_{x}^{\infty}} \lesssim\|\partial \phi(0)\|_{H_{x}^{s-1}, \quad s>s_{0}}
$$

In view of the trivial Cauchy-Schwartz estimate

$$
\|\partial \phi\|_{L_{[0, T]}^{1} L_{x}^{\infty}} \leq T^{\frac{3}{4}}\|\partial \phi\|_{L_{[0, T]}^{4} L_{x}^{\infty}}
$$

and of the energy estimate (3), the proof of (10) is reduced to the following bootstrap argument:

Theorem 2.1 If $\phi$ is a solution of $(1), \phi \in C\left([0, T], H^{s}\right) \cap C^{1}\left([0, T], H^{s-1}\right)$, with $T \leq 1$ and satisfying

$$
\|\partial \phi\|_{L_{[0, T]}^{4} L_{x}^{\infty}}+\|\partial \phi\|_{L_{[0, T]}^{\infty} H_{x}^{s-1}} \leq R
$$

then it will also verify

$$
\|\partial \phi\|_{L_{[0, T]}^{4} L_{x}^{\infty}} \leq C(R) T^{s-s_{0}}\|\partial \phi\|_{L_{[0, T]}^{\infty} H_{x}^{s-1}}
$$

\subsection{Paradifferential approximation and linearization of the prob- lem}

In order to prove (12), we will use a Littlewood-Paley decomposition. In case of the low frequencies the required estimate follows trivially applying the Sobolev inequality. The delicate problem is in the case of the high frequencies $\lambda \geq \Lambda$, where apart from the truncation

\footnotetext{
${ }^{2}$ Throughout this paper we will use the notation $A \lesssim B$ for $A \leq C \cdot B$ where $C$ is a universal constant.
} 
of the coefficients caused by the commutation of the wave operator $\square_{g(\phi)}$ with the LittlewoodPaley operator $P_{\lambda}$, we will further truncate them up to frequency $\lambda^{a}, a \in(0,1)$, using the operator

$$
S_{\lambda^{a}}=\sum_{\mu<\lambda^{a}} P_{\mu}
$$

We introduce the smooth metric

$$
g_{\leq \lambda^{a}}^{i j}=S_{\lambda^{a}} g^{i j}\left(S_{\lambda^{a}} \phi\right) .
$$

The inhomogeneous equation satisfied by the dyadic piece $\phi^{\lambda}$ is then the following one:

$$
\begin{aligned}
& \bar{\square}_{g_{\leq \lambda} a} \phi^{\lambda}=-\partial_{t}^{2} \phi^{\lambda}+g_{\leq \lambda^{a}}^{i j} \partial_{i} \partial_{j} \phi^{\lambda}=R_{\lambda}^{a}, \\
& \left.\phi^{\lambda}\right|_{t=0}=\phi_{0}^{\lambda},\left.\quad \partial_{t} \phi^{\lambda}\right|_{t=0}=\phi_{1}^{\lambda} .
\end{aligned}
$$

The right-hand side $R_{\lambda}^{a}$ has the Fourier support contained in the set

$$
\{\xi: \lambda \leq|\xi| \leq 4 \lambda\}
$$

and satisfies

$$
\left\|R_{\lambda}^{a}(t)\right\|_{\dot{H}^{s}} \lesssim C(R) \lambda^{1-a} c_{\lambda}\|\partial \phi(t)\|_{L_{x}^{\infty}}\|\phi(t)\|_{\dot{H}^{s}}
$$

for any $s>1$ and $t \in[0, T]$, the constants $c_{\lambda}$ verifying $\sum_{\lambda} c_{\lambda}^{2} \leq 1$. Theorem 2.1 is then an immediate consequence of the following

Proposition 2.2 If $\phi$ satisfies the assumptions of Theorem 2.1, then for each $\lambda \geq \Lambda$, where $\Lambda$ is a fixed large parameter, the following Strichartz estimate is true:

$$
\left\|\partial \phi^{\lambda}\right\|_{L_{[0, T]}^{4} L_{x}^{\infty}} \leq C(R) c_{\lambda} T^{s-s_{0}}\|\partial \phi\|_{L_{[0, T]}^{\infty} H_{x}^{s-1}}
$$

for constants $c_{\lambda}$ verifying $\sum_{\lambda} c_{\lambda}^{2} \leq 1$.

For more details we refer the reader to Proposition 1.2 and Theorem 1.3 in [7].

Remark From now on, we can regard (14) as a linear equation with smooth coefficients, depending on the parameter $\lambda$.

\subsection{Restriction to frequency dependent time intervals}

In this part we will reduce the proof of Proposition 2.2 to the proof of precise Strichartz estimates (without losses) on small time intervals. Using the bootstrap assumption (11)

$$
\|\partial \phi\|_{L_{[0, T]}^{4} L_{x}^{\infty}} \leq R
$$

we will partition the time interval $[0, T]$ into smaller intervals $I$ such that they satisfy the following three conditions: 
- their total number is comparable to $\lambda^{1-a}$;

- the size of each $I$ is bounded by $T \lambda^{-(1-a)}$;

- for each $I$

$$
\|\partial \phi\|_{L_{I}^{4} L_{x}^{\infty}} \leq \lambda^{-\frac{1-a}{4}}\|\partial \phi\|_{L_{[0, T]}^{4} L_{x}^{\infty}} \leq R \lambda^{-\frac{1-a}{4}} .
$$

Estimate (16) will then follow as a result of summing the exact Strichartz estimates over the intervals $I$. We will use Duhamel formula to work from now on with an homogeneous equation. We choose $a$ to satisfy ${ }^{3}$

$$
0<a=\frac{8-4 s}{1-4 s+4 s_{0}}<\sqrt{22}-4<1 .
$$

The precise Strichartz estimate to be proved is:

$$
\left\|P_{\lambda} \partial \psi\right\|_{L_{I}^{4} L_{x}^{\infty}} \leq C(R)|I|^{\epsilon}\|\psi[0]\|_{\dot{H}^{\frac{7}{4}+\epsilon}},
$$

where $\psi$ is a solution of the linear wave equation

$$
\square_{g_{\leq \lambda} a} \psi=0
$$

with initial data $\psi[0]$ such that

$$
\text { supp } \hat{\psi}[0] \in\left\{\frac{1}{2} \lambda \leq|\xi| \leq 2 \lambda\right\}
$$

and

$$
\epsilon=s-s_{0} .
$$

The metric $g_{\leq \lambda^{a}}$ verifies for all nonnegative integers $m$ and for all subintervals $I$ :

$$
\begin{aligned}
& \left\|\partial^{1+m} g_{\leq \lambda^{a}}\right\|_{L_{I}^{1} L_{x}^{\infty}} \leq \lambda^{-(1-a)+a m} \bar{R} \\
& \left\|\partial^{1+m} g_{\leq \lambda^{a}}\right\|_{L_{I}^{2} L_{x}^{\infty}} \leq \lambda^{-\frac{1-a}{2}+a m} \bar{R}, \\
& \left\|\partial^{1+m} g_{\leq \lambda^{a}}\right\|_{L_{I}^{4} L_{x}^{\infty}} \leq \lambda^{-\frac{1-a}{4}+a m} \bar{R} \\
& \left\|\partial^{1+m} g_{\leq \lambda^{a}}\right\|_{L_{I}^{\infty} L_{x}^{\infty}} \leq \lambda^{\frac{a^{2}}{4}+a m} \bar{R} \\
& \left\|\partial_{x}^{m}\left(\partial^{2} g_{\leq \lambda^{a}}\right)\right\|_{L_{I}^{\infty} L_{x}^{2}} \leq \lambda^{\frac{a^{2}}{4}+a m} \bar{R}, \\
& \left\|\partial^{m} \bar{\square}_{g_{\leq \lambda^{a}}} g_{\leq \lambda^{a}}\right\|_{L_{I}^{1} L_{x}^{\infty}} \leq \lambda^{-(1-a)+a m} \bar{R}
\end{aligned}
$$

$\bar{R}$ depending only on the constants $M_{0}$ and $R$.

Remarks 1. The estimates (19)-(21) follow immediately as a result of applying Hölder inequality to the estimate (17).

2. The estimates (22)-(23) are deduced using Sobolev inequalities, while (24) appears in view

\footnotetext{
${ }^{3}$ The particular choice for $\sqrt{22}-4$ will become later explicit.
} 
of the fact that $\square_{g} g^{i j}$ verifies (7). It is very easy to check that our linear wave equation $\square_{g_{\leq \lambda} a} \psi=0$ is invariant under rescaling. Introducing

$$
\begin{aligned}
& g_{\lambda}(t, x)=g_{\leq \lambda^{a}}\left(\lambda^{-1} t, \lambda^{-1} x\right), \\
& \varphi(t, x)=\psi\left(\frac{t}{\lambda}, \frac{x}{\lambda}\right),
\end{aligned}
$$

the estimate (18) is then further reduced to the proof of

Proposition 2.3 If $\varphi$ is a solution of the homogeneous equation:

$$
\begin{aligned}
& \square_{g_{\lambda}} \varphi=-\partial_{t}^{2} \varphi+g_{\lambda}^{i j} \partial_{i} \partial_{j} \varphi=0, \\
& \left.\varphi\right|_{t=0}=\varphi_{0},\left.\quad \partial_{t} \varphi\right|_{t=0}=\varphi_{1}
\end{aligned}
$$

on the time interval $I=\left[0, t_{*}\right]$ with $t_{*} \leq \lambda^{a}$ and the metric $g_{\lambda}$ verifies the scaled versions of (19)-(24):

$$
\begin{aligned}
\left\|\partial^{1+m} g_{\lambda}\right\|_{L_{I}^{1} L_{x}^{\infty}} \lesssim \lambda^{-(1-a)(m+1)} \\
\left\|\partial^{1+m} g_{\lambda}\right\|_{L_{I}^{2} L_{x}^{\infty}} \lesssim \lambda^{-\frac{2-a}{2}-(1-a) m} \\
\left\|\partial^{1+m} g_{\lambda}\right\|_{L_{I}^{4} L_{x}^{\infty}} \lesssim \lambda^{-\frac{4-a}{4}-(1-a) m} \\
\left\|\partial^{1+m} g_{\lambda}\right\|_{L_{I}^{\infty} L_{x}^{\infty}} \lesssim \lambda^{-\bar{a}-(1-a) m} \\
\left\|\partial^{2+m} g_{\lambda}\right\|_{L_{I}^{\infty} L_{x}^{2}} \lesssim \lambda^{-\bar{a}-(1-a) m} \\
\left\|\partial^{m} \square_{g_{\lambda}} g_{\lambda}\right\|_{L_{I}^{1} L_{x}^{\infty}} \lesssim \lambda^{-(2-a)-(1-a) m}
\end{aligned}
$$

where $\bar{a}=1-\frac{a^{2}}{4}$ and the parameter a such that $a<\sqrt{22}-4$, then the following Strichartz estimate holds true

$$
\|P \partial \varphi\|_{L_{I}^{4} L_{x}^{\infty}} \lesssim\left|t_{*}\right|^{\epsilon}\|\varphi[0]\|_{L_{x}^{2}}
$$

$P$ is the operator of projection on the set $\left\{\xi: \frac{1}{2} \leq|\xi| \leq 2\right\}$ in Fourier space and the inequality holds with a constant independent of $\lambda$.

Remark: Compared to the case $n=3$, we notice the presence of inequality (29). Note also that (30)-(31) are different from the corresponding inequalities in [7], due to the different scaling in dimension $n=2$.

\subsection{Reduction of the Strichartz estimate to the dispersive in- equality}

This part contains three steps and follows identically the corresponding structure in [7]:

1. Equation (26) can be replaced with the geometric wave equation 


$$
\square_{g_{\lambda}} \phi=-\frac{1}{\sqrt{\operatorname{det} g_{\lambda}}} \partial_{t}\left(\sqrt{\operatorname{det} g_{\lambda}} \partial_{t} \phi\right)+\frac{1}{\sqrt{\operatorname{det} g_{\lambda}}} \partial_{i}\left(g_{\lambda}^{i j} \sqrt{\operatorname{det} g_{\lambda}} \partial_{j} \phi\right)=0
$$

and this is due to the fact that the two wave operators differ only by lower order terms in so far as the Strichartz estimates are concerned.

2. Using a modified version of the standard $T T^{*}$ argument as in [6],[7], we can show that the proof of the Strichartz estimate (33) is reduced to the proof of the following dispersive inequality:

Theorem 2.4 Under the hypothesis of Proposition 2.3, if $\phi$ is a solution of the linear wave equation

$$
\begin{aligned}
& \square_{g_{\lambda}} \phi=0, \\
& \left.\phi\right|_{t=0}=\phi_{0},\left.\quad \partial_{t} \phi\right|_{t=0}=\phi_{1}
\end{aligned}
$$

with initial data $\phi[0]$ supported in the set $\left\{\xi: \frac{1}{2} \leq|\xi| \leq 2\right\}$ in Fourier space, then for all $t \leq t_{*}$ and a fixed arbitrary small $\epsilon>0$

$$
\|P \partial \phi(t)\|_{L_{x}^{\infty}} \lesssim \frac{1}{(1+|t|)^{\frac{1}{2}-\epsilon}}\|\phi[0]\|_{L_{x}^{1}}
$$

3. Considering the same partition of unity as in [7], together with the additivity of the $L^{1}$ norm and the standard Sobolev inequality, we will decompose the initial data $\phi[0]$ into a sum of functions with almost disjoint supports contained in balls of radius $\frac{1}{2}$ and therefore reduce the dispersive inequality (36) to the following $L^{2}-L^{\infty}$ decay estimate:

Theorem 2.5 Under the hypothesis of Theorem 2.4, but with the initial data $\phi\left[t_{0}\right]$ supported in the ball $B_{\frac{1}{2}}(0)$, for all $t_{0} \leq t \leq t_{*}$, an arbitrary small $\epsilon>0$, and a sufficiently large integer $m>0$,

$$
\|P \partial \phi(t)\|_{L_{x}^{\infty}} \lesssim \frac{1}{\left(1+\left|t-t_{0}\right|\right)^{\frac{1}{2}-\epsilon}} \sum_{k=1}^{m}\left\|\partial^{k} \phi\left[t_{0}\right]\right\|_{L_{x}^{2}} .
$$

Remark We will postpone the last step of the reduction until we have all the geometrical ingredients needed in its proof.

\section{The geometrical background of the problem}

\subsection{Basic geometric tools}

From now on, in order to simplify notation, we will denote our underlying metric $g_{\lambda}^{\alpha \beta}=g^{\alpha \beta}$. Therefore we will work with the space-time Lorentz metric

$$
g_{\alpha \beta} d x^{\alpha} d x^{\beta}=-d t^{2}+g_{i j} d x^{i} d x^{j} .
$$


An immediate consequence of this is the fact that $T=\partial_{t}$ is geodesic $\left(\mathcal{D}_{T} T=0\right)$. We will begin with the following

Definition 3.1 1.The spacelike hypersurfaces $\Sigma_{t}$ are defined as the level hypersurfaces of the time function $t$. The time axis $\Gamma$ is the integral curve of the unit vectorfield $T=\partial_{t}$, initiating at the origin.

2. The solution $u$ of the eikonal equation:

$$
g^{\alpha \beta} \partial_{\alpha} u \partial_{\beta} u=0 \quad u\left(\Gamma \cap \Sigma_{t}\right)=t
$$

is called the optical function. In connection with $u$, we will also define

$$
\underline{u}=2 t-u
$$

and

$$
s=t-u \text {. }
$$

$C_{u}$ denotes the level surfaces of function $u$, which are null cones with vertices on $\Gamma$. We define by

$$
L^{\prime}=-g^{\alpha \beta} \partial_{\alpha} u \partial_{\beta}
$$

the normal to the hypersurface $C_{u}$.

3. The function $b$ given by

$$
b^{-1}=-<L^{\prime}, \partial_{t}>=\partial_{t} u
$$

is called the lapse function.

4. The collection of the following vectorfields

$$
L=b L^{\prime} \quad \underline{L}=2 T-L,
$$

together with an unit vectorfield $A$, tangent to $S_{t, u}=\Sigma_{t} \cap C_{u}$, is called a null frame. Regarding $S_{t, u}$ as embedded in $\Sigma_{t}$, we define the unit outward normal $N$.

5. The conformal energy for a function $\phi=\phi(t, x)$ in the interior region and the exterior region is given respectively by

$$
\begin{aligned}
& E_{\text {int }}(\phi)(t)=\int_{\Sigma_{t}}\left(t^{2} \cdot|\partial \phi|^{2}+|\phi|^{2}\right) \cdot(1-\omega) \\
& E_{\text {ext }}(\phi)(t)=\int_{\Sigma_{t}}\left(t^{2} \cdot\left(|L \phi|^{2}+\left(|A \phi|^{2}\right)+u^{2} \cdot|\underline{L} \phi|^{2}+|\phi|^{2}\right) \cdot \omega\right.
\end{aligned}
$$

where $\omega$ is a cut-off function equal to 1 in the region $\left\{u \leq \frac{t}{2}\right\}$, whose derivative satisfies $|\partial \omega| \lesssim t^{-1}$.

The full conformal energy is given by

$$
E(\phi)(t)=E_{\text {int }}(\phi)(t)+E_{\text {ext }}(\phi)(t)
$$


6. We introduce the following special vectorfields:

$$
\begin{aligned}
& \Omega=s \cdot A \quad \text { angular momentum vector field } \\
& S=\frac{1}{2}(u \underline{L}+\underline{u} L) \quad \text { scalar vector field } \\
& K=\frac{1}{2}\left(u^{2} \underline{L}+\underline{u}^{2} L\right) \quad \text { Morawetz vector field }
\end{aligned}
$$

The following facts are simple consequences of the previous definition:

Proposition 3.2 1. $\langle L, L>=<\underline{L}, \underline{L}>=<L, A>=<\underline{L}, A>=0$,

$<L, \underline{L}>=-2, \quad<A, A>=1$.

2. The function $s$ defined by $s=t-u$ is the affine parameter of the vectorfield $L$.

3. $L u=0, \quad L t=L s=1, \quad L \underline{u}=2$.

4. $\underline{L} u=2 b^{-1}, \quad \underline{L t}=1, \quad \underline{L} s=1-2 b^{-1}, \quad \underline{L} \underline{u}=2-2 b^{-1}$.

5. $A u=A t=A s=A \underline{u}=0$.

6. $L=T+N, \underline{L}=T-N$.

We recall the formula for the Ricci tensor

$$
\begin{aligned}
R_{\mu \nu}=g^{\alpha \beta} R_{\alpha \mu \beta \nu} & =\frac{1}{2} g^{\alpha \beta}\left(\partial_{\mu \beta}^{2} g_{\alpha \nu}+\partial_{\alpha \nu}^{2} g_{\mu \beta}-\partial_{\mu \nu}^{2} g_{\alpha \beta}-\partial_{\alpha \beta}^{2} g_{\mu \nu}\right)+ \\
& +g^{\alpha \beta} g_{\gamma \delta}\left(\Gamma_{\mu \beta}^{\gamma} \Gamma_{\alpha \nu}^{\delta}-\Gamma_{\mu \nu}^{\gamma} \Gamma_{\alpha \beta}^{\delta}\right)
\end{aligned}
$$

Relying on the previous proposition, in the particular case of $R_{L L}=R_{L A L A}$, we have the following remarkable decomposition

Proposition 3.3 ([7])

$$
R_{L L}=L(v)-\frac{1}{2} L^{\alpha} L^{\beta} \square_{g} g_{\alpha \beta}+E^{\prime}
$$

the terms

$$
v=L^{\nu} g^{\alpha \beta} \partial_{\beta} g_{\alpha \nu}-\frac{1}{2} g^{\alpha \beta} L\left(g_{\alpha \beta}\right)
$$

and $E^{\prime}$ satisfy the following bounds:

$$
|v| \lesssim|\partial g|, \quad\left|E^{\prime}\right| \lesssim|\partial g|^{2}
$$

An immediate consequence of this proposition is the following

Corollary 3.4 The quantities $v$ and

$$
E=-\frac{1}{2} L^{\alpha} L^{\beta} \square_{g} g_{\alpha \beta}+E^{\prime}
$$


satisfy the estimates:

$$
\begin{aligned}
& |v| \lesssim \lambda^{-\bar{a}} \\
& \int_{0}^{s} \sup _{S_{\rho+u, u}}|E| d \rho \lesssim \lambda^{a-2}
\end{aligned}
$$

Proof The first estimate for $v$ is straightforward due to the trivial bound $|v| \lesssim|\partial g|$ and the condition (30). For $\mathrm{E}$ we have the bound

$$
|E| \lesssim|\partial g|^{2}+\left|\square_{g} g\right|
$$

Therefore using (28) and (32), we obtain:

$$
\int_{0}^{s} \sup _{S_{\rho+u, u}}|E| d \rho \lesssim\|\partial g\|_{L_{\left[0, t_{*}\right]}^{2} L_{x}^{\infty}}^{2}+\left\|\square_{g} g\right\|_{L_{\left[0, t_{*}\right]}^{1} L_{x}^{\infty}} \lesssim \lambda^{a-2} .
$$

We will define now the Ricci coefficients and use them to describe the Levi-Civitta connection defined by $g$.

Theorem 3.5 We define the following tensors on $S_{t, u}$ :

$$
\begin{gathered}
\chi=<\mathcal{D}_{A} L, A>, \quad \underline{\chi}=<\mathcal{D}_{A} \underline{L}, A>, \\
\eta=\frac{1}{2}<\mathcal{D}_{\underline{L}} L, A>, \quad \underline{\eta}=\frac{1}{2}<\mathcal{D}_{L} \underline{L}, A>, \\
\underline{\xi}=\frac{1}{2}<\mathcal{D}_{\underline{L}} \underline{L}, A>.
\end{gathered}
$$

Based on these, the connection $\mathcal{D}$ satisfies the following equations:

$$
\begin{aligned}
& \mathcal{D}_{L} L=-\kappa_{N N} L, \quad \mathcal{D}_{L} \underline{L}=2 \underline{\eta} A+\kappa_{N N} \underline{L}, \quad \mathcal{D}_{L} A=\underline{\eta} L . \\
& \mathcal{D}_{\underline{L}} L=2 \eta A+\kappa_{N N} L, \quad \mathcal{D}_{\underline{L}} \underline{L}=2 \underline{\xi} A-\kappa_{N N} \underline{L}, \quad \mathcal{D}_{\underline{L}} A=\eta \underline{L}+\underline{\xi} L . \\
& \mathcal{D}_{A} L=\chi A-\kappa_{A N} L, \quad \mathcal{D}_{A} \underline{L}=\underline{\chi} A+\kappa_{A N} \underline{L}, \quad \mathcal{D}_{A} A=\frac{1}{2} \chi \underline{L}+\frac{1}{2} \underline{\chi} L .
\end{aligned}
$$

Moreover:

$$
\begin{gathered}
\underline{\chi}=-\chi-2 \kappa_{A A}, \\
\eta=b^{-1} \not \bar{\not}(b)+\kappa_{A N}, \quad \underline{\eta}=-\kappa_{A N}, \\
\underline{\xi}=\kappa_{A N}-\eta .
\end{gathered}
$$


Remarks 1) The proof of this theorem follows exactly the same lines as the corresponding result in [7], being just a simple application of the orthogonalities of the null frame $\{L, \underline{L}, A\}$ and of the eikonal equation (38) together with the basic properties of the Levi-Civitta connection.

2) We notice that we can express all the other Ricci coefficients in terms of just $\chi$, $\eta$, the lapse function $b$ and the second fundamental form $\kappa$.

3) We notice also that due to the fact $S_{t, u}$ is a 1-dimensional surface, all our Ricci coefficients can be treated as scalars. Therefore, if we denote by $\mathbb{\nabla}$ the induced covariant derivative on $S_{t, u}$, the A-derivative of a Ricci coefficient will coincide with its $\not \nabla$-derivative.

Corollary 3.6 We have the following commutation properties:

$$
\begin{aligned}
& {[L, \underline{L}]=2(\underline{\eta}-\eta) A+\kappa_{N N} \underline{L}-\kappa_{N N} L,} \\
& {[L, A]=-\chi A,} \\
& {[\underline{L}, A]=-\underline{\chi} A+\left(\eta-\kappa_{A N}\right) \underline{L}+\underline{\xi} L .}
\end{aligned}
$$

Among the tools that play an important role in the proof of the asymptotic properties are the transport and the elliptic equations satisfied by the different Ricci coefficients. The ones that will be used extensively are:

Lemma 3.7 The following transport and elliptic equations hold:

$$
\begin{aligned}
& L(\chi)=-(\chi)^{2}-\kappa_{N N} \chi-R_{L L}, \\
& L(\eta)=-\chi \eta+\chi \underline{\eta}+\frac{1}{2} R_{A L L \underline{L}}, \\
& L(b)=-b \kappa_{N N}, \\
& \underline{L}(\chi)-(\chi)^{2}-\left(\kappa_{N N}+2 \kappa_{A A}\right) \chi=2 \not \nabla(\eta)+2 \eta^{2}+R_{A L \underline{L A}} .
\end{aligned}
$$

Proof In this proof we use the equations for connection $\mathcal{D}$ and basic formulae for the curvature tensor. In what concerns the transport equations, we present the proof for (63), the other two equations being deduced similarly. Using (53) and taking an $L$-derivative we can write

$$
\begin{aligned}
L(\eta)= & \frac{1}{2}<\mathcal{D}_{L} \mathcal{D}_{\underline{L}} L, A>+\frac{1}{2}<\mathcal{D}_{\underline{L}} L, \mathcal{D}_{L} A>=\frac{1}{2}<\mathcal{D}_{\underline{L}} \mathcal{D}_{L} L, A>+ \\
& +\frac{1}{2}<\mathcal{D}_{[L, \underline{L}]} L, A>+\frac{1}{2}<R_{L \underline{L}} L, A>+\frac{1}{2}<\mathcal{D}_{\underline{L}} L, \mathcal{D}_{L} A>
\end{aligned}
$$

If we plug in the frame equations (55), (56), (57) and the commutation formula (61), we obtain (63). 
The deduction of the elliptic estimate (65) follows like this:

$$
\begin{aligned}
& \underline{L}(\chi)=<\mathcal{D}_{\underline{L}} \mathcal{D}_{A} L, A>+<\mathcal{D}_{A} L, \mathcal{D}_{\underline{L}} A>=R_{A L \underline{L} A}+<\mathcal{D}_{A} \mathcal{D}_{\underline{L}} L, A>+ \\
& \quad+<\mathcal{D}_{[\underline{L}, A]} L, A>+<\mathcal{D}_{A} L, \mathcal{D}_{\underline{L}} A>=R_{A L \underline{L} A}+<\mathcal{D}_{A}\left(2 \eta A+\kappa_{N N} L\right), A> \\
& \quad+<\mathcal{D}_{-\underline{\chi} A+\left(\eta-\kappa_{A N}\right) \underline{L}+\underline{\xi} L} L, A>+<\chi A-\kappa_{A N} L, \eta \underline{L}+\underline{\xi} L>= \\
& \quad=R_{A L \underline{L} A}+2 \not \nabla(\eta)+\kappa_{N N} \chi-\underline{\chi} \chi+2\left(\eta-\kappa_{A N}\right) \eta+2 \eta \kappa_{A N} .
\end{aligned}
$$

Corollary 3.8 If we write the elliptic equation (65) in the form

$$
\not(\eta)+\eta^{2}=H
$$

we have the following estimate

$$
\|\not \eta\|_{L^{2}\left(S_{t, u}\right)}+\|\eta\|_{L^{4}\left(S_{t, u}\right)}^{2} \lesssim\|H\|_{L^{2}\left(S_{t, u}\right)}
$$

Proof We square equation (66) and integrate the resulting expression over $S_{t, u}$. Due to the fact that $\int_{S_{t, u}} \not(\eta) \eta^{2}=0$, we obtain the identity

$$
\int_{S_{t, u}}\left(|\not \nabla|^{2}+|\eta|^{4}\right)=\int_{S_{t, u}} H^{2}
$$

which implies (67).

\subsection{Construction of the optical function $\mathrm{u}$ and the continuation argument}

We review here the main steps in the construction of $u$ discussed in [7]. It will be, of course, enough to describe its level hypersurfaces $C_{u}$, which are the union of null geodesics $x=x(s)$, starting on the time axis $\Gamma$ from the vertex $(u, 0)$ and having velocity in the direction of the vector $(1, \varpi)$, with $\varpi \in \mathcal{S}^{1}$. Relative to our Lorentz metric $g$, the equations for such a geodesic is:

$$
\begin{aligned}
& \frac{d^{2} x^{i}}{d s^{2}}+\Gamma_{j k}^{i} \frac{d x^{j}}{d s} \frac{d x^{k}}{d s}=0 \quad(i, j, k=0, \ldots, 2), \\
& \frac{d x}{d s}(0)=(1, \varpi) .
\end{aligned}
$$

Obviously, these equations can then be rewritten in the form of a first order system for 6 dependent variables, and so, due to the basic existence theorem for ordinary differential equations, we obtain a local solution. We are interested up to what value of $s$ such a local solution can be extended to. Heuristically, such a system can be approximated by the following ODE :

$$
\frac{d y}{d s}+\Gamma(s) y^{2}=0
$$


A local solution can then be continued up to the value of the parameter $s=s_{\max }$, as long as, for example,

$$
s_{\max }|\Gamma(s)| \ll 1 .
$$

Due to the bound that we have for $\left|\Gamma_{j k}^{i}\right|$,

$$
\left|\Gamma_{j k}^{i}\right| \lesssim|\partial g| \lesssim \lambda^{-\bar{a}}
$$

and the fact that

$$
t_{*} \lambda^{-\bar{a}} \lesssim \lambda^{a-\bar{a}} \ll 1
$$

we can argue that such a geodesic can be extended up to the value of the affine parameter $s=t_{*}-u$. It can be shown that the transport equation (64) for the lapse function $b$ implies that our geodesic intersect each time slice $\Sigma_{t}, t \leq t_{*}$. From the definitions, initial condition of the optical function $u$ and the geometry around the time axis $\Gamma$, we can check the following Initial values For all $t \in\left[0, t_{*}\right]$, there exists a constant $R_{0}>0$ such that:

$$
\begin{aligned}
& \limsup _{s \longrightarrow 0}\left(\left|\chi-\frac{1}{s}\right|+|\eta(s)|\right)<R_{0}, \\
& \limsup _{s \longrightarrow 0} s^{\frac{1}{2}}\left(\left\|\mathcal{D}\left(\chi-\frac{1}{s}\right)\right\|_{L^{2}\left(S_{t, u}\right)}+\|\not(\eta)\|_{L^{2}\left(S_{t, u}\right)}\right)<R_{0}, \\
& \lim _{s \rightarrow 0}\left(|b(s)-1|+\left|A\left(S_{t, u}\right)-2 \pi s\right|\right)=0 \\
& \lim _{s \rightarrow 0}\left(s^{3}\left|\mathcal{D}\left(\chi-\frac{1}{s}\right)\right|+s^{2}|\not \nabla \eta(s)|\right)=0 .
\end{aligned}
$$

We will rely on a continuation argument, used also in [7], whose essence we explain in the next lines. We denote by $s(t)$ the maximum value of $s=t-u$, for which the above initial values can be extended in the neighborhood of $\Gamma$ with the additional condition $s(t) \leq \min \left\{\varepsilon R_{0}^{-1}, t\right\}$ where $\varepsilon \ll 1$. So in this region that we denote by $\Delta \subset\left[0, t_{*}\right] \times \mathbb{R}^{2}$, the following are true:

\section{Bootstrap assumptions}

$$
\begin{aligned}
& \left|\chi-\frac{1}{s}\right|+|\eta(s)| \leq R_{0}, \\
& s^{\frac{1}{2}}\left(\left\|\mathcal{D}\left(\chi-\frac{1}{s}\right)\right\|_{L^{2}\left(S_{t, u}\right)}+\|\not \nabla(\eta)\|_{L^{2}\left(S_{t, u}\right)}\right) \leq R_{0}, \\
& s(t) \leq \min \left\{\varepsilon R_{0}^{-1}, t\right\} .
\end{aligned}
$$

We will show that under these assumptions, $R_{0}$ can be chosen such that

$$
\min \left\{\varepsilon R_{0}^{-1}, t\right\}=t
$$

and so from the maximality of $\Delta$ we will conclude that the assumptions will then hold in the whole region

$$
\left\{(s, t) \mid s \in[0, t], t \in\left[0, t_{*}\right]\right\} .
$$

Throughout this section and the following one, we will prove estimates in the region $\Delta$. 


\subsection{Other geometric results}

Based on the bootstrap assumptions (70)-(72), just as in [7], we can prove the following result:

Proposition 3.9 A. Sobolev inequality For any smooth function $f$, $f: S_{t, u} \rightarrow \mathbb{R}, 1<p<\infty$, we have the following Sobolev estimate:

$$
\sup _{S_{t, u}}|f| \lesssim s^{1-\frac{1}{p}}\|\not \nabla f\|_{L^{p}\left(S_{t, u}\right)}+s^{-\frac{1}{p}}\|f\|_{L^{p}\left(S_{t, u}\right)}
$$

B. Trace estimates For any function $h, h: \Sigma_{t} \rightarrow \mathbb{R}, h \in H^{\frac{1}{2}+\epsilon}\left(\mathbb{R}^{2}\right)$ and $\Delta_{s}=\cup_{\frac{1}{4} s \leq \rho \leq s} S_{t, t-\rho}$, we have the following inequalities:

$$
\begin{gathered}
\|f\|_{L^{2}\left(S_{t, u}\right)} \lesssim\left\|\partial^{\frac{1}{2}-\epsilon} f\right\|_{L^{2}\left(\Sigma_{t}\right)}+\left\|\partial^{\frac{1}{2}+\epsilon} f\right\|_{L^{2}\left(\Sigma_{t}\right)} . \\
\|f\|_{L^{2}\left(S_{t, u}\right)}^{2} \lesssim\|N(f)\|_{L^{2}\left(\Delta_{s}\right)}\|f\|_{L^{2}\left(\Delta_{s}\right)}+\frac{1}{s}\|f\|_{L^{2}\left(\Delta_{s}\right)}^{2} .
\end{gathered}
$$

Remark These two results, in a more general form, can be found for the case $n=3$ in [7]. They will also survive in the general form in the case $n=2$, but not to create too much confusion, we presented them in the form perfectly suitable to our situation. We remark also that the proof that we have right now is almost identical with the original one, the only difference with respect to the case $n=3$ being the fact that we are no longer able to recover the isoperimetric inequality. We hope to provide in the future a different proof for (73)-(75), which would rely more on the special structure of $S_{t, u} \cdot{ }^{4}$

The next result will be extensively used in the proof of the asymptotic properties for the Ricci coefficients.

Transport lemma If $\Xi_{\underline{A}}$ is an $S_{t, u}$-tangent covariant tensor satisfying the transport equation

$$
L\left(\Xi_{\underline{A}}\right)+\sigma \chi \Xi_{\underline{A}}=F_{\underline{A}},
$$

with the initial condition $s^{\sigma} \Xi_{\underline{A}}(s) \longrightarrow 0$ as $s \longrightarrow 0$ and $(t, x)=(t, s, \omega) \in \Delta$, the following estimates hold:

If $\sigma>-\frac{1}{2}$ then

$$
|\Xi(t, x)| \leq \frac{4}{s^{\sigma}} \int_{0}^{s} \rho^{\sigma}|F| d \rho
$$

Additionally, if $s^{\sigma-\frac{1}{2}}\|\Xi\|_{L^{2}\left(S_{t, u}\right)} \longrightarrow 0$ as $s \longrightarrow 0$, we have

$$
\|\Xi\|_{L^{2}\left(S_{t, u}\right)} \leq \frac{4}{s^{\sigma-\frac{1}{2}}} \int_{0}^{s} \rho^{\sigma-\frac{1}{2}}\|F\|_{L^{2}\left(S_{u+\rho, u}\right)} d \rho
$$

\footnotetext{
${ }^{4}$ This proof would take advantage of the fact that $S_{t, u}$ is the intersection of the level sets for the functions $t$ and $u$.
} 
If $\sigma \geq 0$ and $F$ defined in the whole time slab $\left[0, t_{*}\right] \times \mathbb{R}^{2}$, we have also

$$
|\Xi(t, x)| \leq 4\|F\|_{L_{\left[0, t_{*}\right]}^{1} L_{x}^{\infty}} .
$$

If $\sigma>0$, it follows that

$$
|\Xi(t, x)| \leq \frac{4}{\sigma} \sup _{\rho \leq s} \rho \cdot|F|
$$

Proof The transport equation (76) implies:

$$
\frac{1}{2} \frac{d}{d \mathbf{s}}|\Xi|^{2}+\frac{\sigma}{s}|\Xi|^{2}=-\sigma\left(\chi-\frac{1}{s}\right)|\Xi|^{2}+F \cdot \Xi
$$

which further yields

$$
\frac{d}{d \mathbf{s}}\left(s^{2 \sigma} \cdot|\Xi|^{2}\right)=-2 \sigma s^{2 \sigma}\left(\chi-\frac{1}{s}\right)|\Xi|^{2}+2 s^{2 \sigma} F \cdot \Xi .
$$

Integrating this equation with respect to $s$ and taking into account the initial condition, we obtain:

$$
|\Xi(t, x)|^{2} \leq \frac{2|\sigma|}{s^{2 \sigma}} \int_{0}^{s} \rho^{2 \sigma}\left|\chi-\frac{1}{s}\right||\Xi|^{2} d \rho+\frac{2}{s^{2 \sigma}} \int_{0}^{s} \rho^{2 \sigma}|F \cdot \Xi| d \rho .
$$

Using now the fact that $\left|\chi-\frac{1}{s}\right| \leq R_{0}$, contained in the assumptions, together with the hypothesis, $\sigma>-\frac{1}{2}$, we can write

$$
\sup _{\rho \leq s}|\Xi|^{2} \leq \frac{2|\sigma|}{2 \sigma+1} R_{0} s \sup _{\rho \leq s}|\Xi|^{2}+\frac{2}{s^{2 \sigma}} \int_{0}^{s} \rho^{2 \sigma}|F \cdot \Xi| d \rho .
$$

Due to the fact that $s \leq s(t)$ and $R_{0} s(t) \ll 1$, by the assumption (72), we can conclude that

$$
\sup _{\rho \leq s}|\Xi|^{2} \leq \frac{4}{s^{2 \sigma}} \int_{0}^{s} \rho^{2 \sigma}|F \cdot \Xi| d \rho
$$

Finally using Gronwall inequality, we end up with

$$
|\Xi| \leq \frac{4}{s^{\sigma}} \int_{0}^{s} \rho^{\sigma}|F| d \rho
$$

This implies of course (77) and (80). (79) follows also immediately. To deduce (78) we will continue from (81) by integrating it over the fixed surface $S_{t, u}$. Due to the fact that $\int_{S_{t, u}} f \approx s \cdot \int_{0}^{2 \pi} f(s, \omega) d \omega$, we obtain

$$
\begin{aligned}
s^{2 \sigma-1}\|\Xi\|_{L^{2}\left(S_{t, u}\right)}^{2} & \leq 4|\sigma| \int_{0}^{s} \rho^{2 \sigma-1}\|\Xi\|_{L^{2}\left(S_{\rho+u, u}\right)}^{2} d \rho+ \\
& +2 \int_{0}^{s} \rho^{2 \sigma-1}\|F\|_{L^{2}\left(S_{\rho+u, u}\right)}\|\Xi\|_{L^{2}\left(S_{\rho+u, u}\right)} d \rho .
\end{aligned}
$$

Here applying twice Gronwall inequality, we come up with the desired result. Finally we present integration results which will be useful in the sections which deal with commutation of the D'Alembertian with the special vectorfields: 
Proposition 3.10 (Integration results) 1. If $f$ and $h$ are smooth functions defined on $\Sigma_{t}$, then the following estimate holds:

$$
\|f \cdot h \cdot \omega\|_{L^{2}\left(\Sigma_{t}\right)} \lesssim t^{-\frac{1}{2}} \sup _{0 \leq u \leq \frac{t}{2}}\|h\|_{L^{2}\left(S_{t, u}\right)} \cdot E^{\frac{1}{2}}(f)(t)
$$

2. In the context of our reduced linear problem, we can write the following integration by parts:

$$
\int_{\left[t_{0}, t\right] \times \mathbb{R}^{2}} V \cdot L \psi \cdot \underline{L} \psi \cdot \omega=\int_{\left[t_{0}, t\right] \times \mathbb{R}^{2}} V \cdot \square_{g} \psi \cdot \psi \cdot \omega+O\left(\lambda^{-\epsilon}\right) \sup _{\tau \in\left[t_{0}, t\right]} E(\psi)(\tau)
$$

provided the following conditions hold

$$
\begin{aligned}
& \|V\|_{L^{\infty}\left(S_{\tau, u}\right)} \lesssim \tau \cdot \lambda^{-\epsilon} \\
& \int_{t_{0}}^{t} \tau^{-\frac{3}{2}} \sup _{0 \leq u \leq \frac{\tau}{2}}\left(\|\underline{L} V\|_{L^{2}\left(S_{\tau, u}\right)}+\|\not \nabla V\|_{L^{2}\left(S_{\tau, u}\right)}\right) d \tau \lesssim \lambda^{-\epsilon}
\end{aligned}
$$

Also we have the estimate:

$$
\left|\int_{\left[t_{0}, t\right] \times \mathbb{R}^{2}} V \cdot \psi \cdot \underline{L} \psi \cdot \omega\right| \lesssim \lambda^{-\epsilon} \sup _{\tau \in\left[t_{0}, t\right]} E(\psi)(\tau)
$$

if the previous conditions hold, relaxed by the fact that we can drop the term $\|\not \nabla V\|_{L^{2}\left(S_{\tau, u}\right)}$. Remark The proof of these integration formulae is straightforward, as in the case of their corresponding ones for $n=3$, presented in [r].

Now we are ready to prove

\section{Asymptotics properties of the Ricci coefficients}

The main result of this section is:

Theorem 4.1 For sufficiently large values of $\lambda$ and for the parameter a verifying $a<\sqrt{22}-4$, the Ricci coefficients $\chi$, $\eta$, any component $R$ of the curvature, the lapse function $b$ and the second fundamental form $\kappa$ satisfy the following estimates:

$$
\begin{aligned}
& \sup _{S_{t, u}}\left|\chi-\frac{1}{s}\right|+\sup _{S_{t, u}}|\kappa|+\sup _{S_{t, u}}|L(b)| \lesssim \lambda^{-\bar{a}} \\
& \sup _{S_{t, u}}|\eta|+\sup _{S_{t, u}}|\not|(b) \mid \lesssim s^{\frac{1}{2}} \lambda^{-\bar{a}-\frac{1-a}{2}+\epsilon}+\lambda^{-\bar{a}} \\
& \sup _{S_{t, u}}|\eta| \lesssim \min \left\{s \lambda^{-\bar{a}-(1-a)}+\lambda^{-\bar{a}}, s^{\frac{1}{2}} \lambda^{-\bar{a}-\frac{1-a}{2}+\epsilon}+\lambda^{-\bar{a}}\right\} \\
& \sup _{S_{t, u}}|b-1| \lesssim \min \left\{s \lambda^{-\bar{a}}, \lambda^{-(1-a)}\right\} \\
& \sup _{S_{t, u}}|\underline{L}(b)| \lesssim s \lambda^{-\bar{a}-(1-a)}
\end{aligned}
$$




$$
\begin{aligned}
& \|R\|_{L^{2}\left(S_{t, u}\right)}+\|\nabla \kappa\|_{L^{2}\left(S_{t, u}\right)} \lesssim \lambda^{-\bar{a}-\frac{1-a}{2}+\epsilon} \\
& \left\|\left|L\left(\kappa_{A \cdot}\right)\right|+\left|L\left(\kappa_{N \cdot}\right)\right|\right\|_{L^{2}\left(S_{t, u}\right)}+\left\|\left|\underline{L}\left(\kappa_{A \cdot}\right)\right|+\left|\underline{L}\left(\kappa_{N \cdot}\right)\right|\right\|_{L^{2}\left(S_{t, u}\right)} \lesssim \lambda^{-\bar{a}-\frac{1-a}{2}+\epsilon} \\
& \left\|\left|\not \nabla\left(\kappa_{A}\right)\right|+\left|\not \nabla\left(\kappa_{N \cdot}\right)\right|\right\|_{L^{2}\left(S_{t, u}\right)} \lesssim \lambda^{-\bar{a}-\frac{1-a}{2}+\epsilon}+s^{-\frac{1}{2}} \lambda^{-\bar{a}} \\
& \left\|\mathcal{D}\left(\chi-\frac{1}{s}\right)\right\|_{L^{2}\left(S_{t, u}\right)}+\|\not \nabla(\eta)\|_{L^{2}\left(S_{t, u}\right)}+\|L(\eta)\|_{L^{2}\left(S_{t, u}\right)} \lesssim \lambda^{-\bar{a}-\frac{1-a}{2}+\epsilon}+s^{-\frac{1}{2}} \lambda^{-\bar{a}} \\
& \|L(b)\|_{L^{2}\left(S_{t, u}\right)} \lesssim s^{\frac{1}{2}} \lambda^{-\bar{a}} \\
& \|\underline{L}(b)\|_{L^{2}\left(S_{t, u}\right)} \lesssim s \lambda^{-\bar{a}-\frac{1-a}{2}+\epsilon} \\
& \|\not D(b)\|_{L^{2}\left(S_{t, u}\right)} \lesssim s \lambda^{-\bar{a}-\frac{1-a}{2}+\epsilon}+s^{\frac{1}{2}} \lambda^{-\bar{a}}
\end{aligned}
$$

First, we will obtain the estimates for the second fundamental form $\kappa$ and for the components $R$ of the curvature, using the bounds

$$
\begin{aligned}
& |\kappa| \lesssim|\partial g| \\
& |R| \lesssim\left|\partial^{2} g\right|+|\partial g|^{2},
\end{aligned}
$$

and the trace estimate (74). Next, we investigate the Ricci coefficient $\chi$. To derive the estimates, we use first the transport equation (62) together with the decomposition (47) of the term $R_{L L}$ in order to infer that:

$$
L\left(\chi-\frac{1}{s}+v\right)+2 \chi\left(\chi-\frac{1}{s}+v\right) \approx E,
$$

where $|v| \lesssim|\partial g|$ and $|E| \lesssim|\partial g|^{2}$ are the terms that appear in the decomposition of $R_{L L}$. Integrating this equation, we will obtain the bound

$$
\sup _{S_{t, u}}\left|\chi-\frac{1}{s}\right| \lesssim \lambda^{-\bar{a}}
$$

and, as an immediate consequence, the estimate:

$$
\left\|L\left(\chi-\frac{1}{s}\right)\right\|_{L^{2}\left(S_{t, u}\right)} \lesssim \lambda^{-\bar{a}-\frac{1-a}{2}+\epsilon}+s^{-\frac{1}{2}} \lambda^{-\bar{a}} .
$$

Using again the special structure of $R_{L L}$, we will then write the transport equation for the quantity $\not\left(\chi-\frac{1}{s}+v\right)$ and apply the Transport lemma in order to obtain the estimate for $\left\|\not \nabla\left(\chi-\frac{1}{s}\right)\right\|_{L^{2}\left(S_{t, u}\right)}$. The estimate of the $\underline{L}$ derivative of $\chi-\frac{1}{s}$ will be obtained in connection with the estimates for $\eta$. The third part of the proof deals with Ricci coefficient $\eta$. If we try to investigate $\eta$ using its transport equation (63), we will obtain the estimate $\sup _{S_{t, u}}|\eta| \lesssim$ $\lesssim \lambda^{2 a-\bar{a}-1}$, which is worse than the one claimed in the theorem. This is due to the fact that the curvature component present in the transport equation for $\eta$ does not have a special decomposition. There is though a better way to estimate $\eta$ and this is with the help of the elliptic equation (65), which we can write in the form:

$$
\not(\eta)+\eta^{2}=H
$$


where $H$ will be evaluated using its transport equation. We will take advantage of the elliptic estimate (67)

$$
\|\not \nabla(\eta)\|_{L^{2}\left(S_{t, u}\right)}+\|\eta\|_{L^{4}\left(S_{t, u}\right)}^{2} \lesssim\|H\|_{L^{2}\left(S_{t, u}\right)}
$$

and the bound that we already have for $\left\|\underline{L}\left(\chi-\frac{1}{s}\right)\right\|_{L^{2}\left(S_{t, u}\right)}$. Thus, we obtain:

$$
\sup _{S_{t, u}}|\eta| \lesssim s^{\frac{1}{2}} \lambda^{-\bar{a}-\frac{1-a}{2}+\epsilon}+\lambda^{-\bar{a}} .
$$

An immediate application of this estimate is the bound for $\|L \eta\|_{L^{2}\left(S_{t, u}\right)}$. We make here two important remarks. The first one is the fact that the estimate for $\eta$ is worse than the one for $\chi-\frac{1}{s}$ and so this estimate is the one which dictates the range for $a$. Secondly, as in the case $n=3$, we do not have a good estimate for $\underline{L} \eta$. Finally, we turn our attention to the lapse function $b$. We notice that estimates for all the quantities depending on $b$, with the exception of $\underline{L} b$, are immediate consequences of previously obtained bounds . For $\underline{L} b$, we write its transport equation and deduce the estimate using once again the Transport lemma.

\subsection{Proof of the estimates for the second fundamental form $\kappa$ and for the components $R$ of the curvature}

The obvious estimate $|\kappa| \lesssim|\partial g|$ implies

$$
\sup _{S_{t, u}}|\kappa| \lesssim\|\partial g\|_{L^{\infty}\left(\Sigma_{t}\right)}
$$

which coupled with (30) gives us the desired estimate. Any component $R$ of the curvature satisfies

$$
|R| \lesssim\left|\partial^{2} g\right|+|\partial g|^{2}
$$

so using the trace estimate (74), we conclude that:

$$
\begin{aligned}
\|R\|_{L^{2}\left(S_{t, u}\right)} & \lesssim\left\|\partial^{2} g\right\|_{L^{2}\left(S_{t, u}\right)}+\left(A\left(S_{t, u}\right)\right)^{\frac{1}{2}}\|\partial g\|_{L^{\infty}\left(\Sigma_{t}\right)}^{2} \\
& \lesssim\left\|\partial^{2+\frac{1}{2}+\epsilon} g\right\|_{L^{2}\left(\Sigma_{t}\right)}+\left\|\partial^{2+\frac{1}{2}-\epsilon} g\right\|_{L^{2}\left(\Sigma_{t}\right)}+\left(A\left(S_{t, u}\right)\right)^{\frac{1}{2}}\|\partial g\|_{L^{\infty}\left(\Sigma_{t}\right)}^{2} \\
& \lesssim \lambda^{-\bar{a}-\frac{1-a}{2}+\epsilon}+s^{\frac{1}{2}} \lambda^{-2 \bar{a}} .
\end{aligned}
$$

Using the bound for $a$ :

$$
s^{\frac{1}{2}} \lambda^{-2 \bar{a}} \lesssim \lambda^{-\bar{a}-\frac{1-a}{2}+\epsilon}
$$

and so, $\|R\|_{L^{2}\left(S_{t, u}\right)} \lesssim \lambda^{-\bar{a}-\frac{1-a}{2}+\epsilon}$. This implies also $\|\nabla \kappa\|_{L^{2}\left(S_{t, u}\right)} \lesssim \lambda^{-\bar{a}-\frac{1-a}{2}+\epsilon}$. We have the following formulae for the derivatives of the second fundamental form $\kappa$ :

$$
\begin{aligned}
& L\left(\kappa_{N N}\right)=\left(\mathcal{D}_{L} \kappa\right)(N, N)+2 \kappa\left(\mathcal{D}_{L} N, N\right)=\left(\mathcal{D}_{L} \kappa\right)(N, N)+2 \kappa_{A N}^{2} \\
& \underline{L}\left(\kappa_{N N}\right)=\left(\mathcal{D}_{\underline{L}} \kappa\right)(N, N)+2 \kappa\left(\mathcal{D}_{\underline{L}} N, N\right)=\left(\mathcal{D}_{\underline{L}} \kappa\right)(N, N)+2\left(2 \eta-\kappa_{A N}\right) \kappa_{A N} \\
& \mathbb{\nabla}\left(\kappa_{N N}\right)=\left(\mathcal{D}_{A} \kappa\right)(N, N)+2 \kappa\left(\mathcal{D}_{A} N, N\right)=\left(\mathcal{D}_{A} \kappa\right)(N, N)+2\left(\chi+\kappa_{A A}\right) \kappa_{A N}
\end{aligned}
$$




$$
\begin{aligned}
& L\left(\kappa_{A A}\right)=\left(\mathcal{D}_{L} \kappa\right)(A, A)+2 \kappa\left(\mathcal{D}_{L} A, A\right)=\left(\mathcal{D}_{L} \kappa\right)(A, A)-2 \kappa_{A N}^{2} \\
& \underline{L}\left(\kappa_{A A}\right)=\left(\mathcal{D}_{\underline{L}} \kappa\right)(A, A)+2 \kappa\left(\mathcal{D}_{\underline{L}} A, A\right)=\left(\mathcal{D}_{\underline{L}} \kappa\right)(A, A)+2\left(\kappa_{A N}-\eta\right) \kappa_{A N} \\
& \not{\nabla}\left(\kappa_{A A}\right)=\left(\mathcal{D}_{A} \kappa\right)(A, A)+2 \kappa\left(\mathcal{D}_{A} A, A\right)=\left(\mathcal{D}_{A} \kappa\right)(A, A)-2\left(\chi+\kappa_{A A}\right) \kappa_{A N} \\
& L\left(\kappa_{A N}\right)=\left(\mathcal{D}_{L} \kappa\right)(A, N)+\kappa\left(\mathcal{D}_{L} A, N\right)+\kappa\left(\mathcal{D}_{L} N, A\right) \\
& =\left(\mathcal{D}_{L} \kappa\right)(A, N)+\kappa_{A N}\left(\kappa_{A A}-\kappa_{N N}\right) \\
& \underline{L}\left(\kappa_{A N}\right)=\left(\mathcal{D}_{\underline{L}} \kappa\right)(A, N)+\kappa\left(\mathcal{D}_{\underline{L}} A, N\right)+\kappa\left(\mathcal{D}_{\underline{L}} N, A\right) \\
& =\left(\mathcal{D}_{\underline{L}} \kappa\right)(A, N)-\left(\kappa_{A A}-\kappa_{N N}\right)\left(\kappa_{A N}-2 \eta\right) \\
& \not \nabla\left(\kappa_{A N}\right)=\left(\mathcal{D}_{A} \kappa\right)(A, N)+\kappa\left(\mathcal{D}_{A} A, N\right)+\kappa\left(\mathcal{D}_{A} N, A\right) \\
& =\left(\mathcal{D}_{A} \kappa\right)(A, N)+\left(\kappa_{A A}-\kappa_{N N}\right)\left(\chi+\kappa_{A A}\right) .
\end{aligned}
$$

We will show the proof for the quantities depending on $\kappa_{N N}$, the ones depending on $\kappa_{A A}$ and $\kappa_{A N}$ following in a similar manner. Using the bootstrap assumptions (70), (72) and the inequality for $\sup _{S_{t, u}}|\kappa|$, by taking the $L^{2}\left(S_{t, u}\right)$ norm in the above formulae we obtain:

$$
\begin{aligned}
&\left\|L\left(\kappa_{N N}\right)\right\|_{L^{2}\left(S_{t, u}\right)} \lesssim \lambda^{-\bar{a}-\frac{1-a}{2}+\epsilon}+s^{\frac{1}{2}} \lambda^{-2 \bar{a}} \lesssim \lambda^{-\bar{a}-\frac{1-a}{2}+\epsilon} \\
&\left\|\underline{L}\left(\kappa_{N N}\right)\right\|_{L^{2}\left(S_{t, u}\right)} \lesssim \lambda^{-\bar{a}-\frac{1-a}{2}+\epsilon}+s^{\frac{1}{2}} \lambda^{-\bar{a}}\left(R_{0}+\lambda^{-\bar{a}}\right) \lesssim \lambda^{-\bar{a}-\frac{1-a}{2}+\epsilon}+s^{-\frac{1}{2}} \lambda^{-\bar{a}} \\
&\left\|\not\left(\kappa_{N N}\right)\right\|_{L^{2}\left(S_{t, u}\right)} \lesssim \lambda^{-\bar{a}-\frac{1-a}{2}+\epsilon}+s^{\frac{1}{2}} \lambda^{-\bar{a}}\left(R_{0}+\frac{1}{s}+\lambda^{-\bar{a}}\right) \lesssim \lambda^{-\bar{a}-\frac{1-a}{2}+\epsilon}+s^{-\frac{1}{2}} \lambda^{-\bar{a}} .
\end{aligned}
$$

We notice that for the $\underline{L}$ derivatives the estimates are not the ones that we claimed, but as soon as we have the estimate for $\eta$, we will deduce them immediately.

\subsection{Proof of the estimates for $\chi-\frac{1}{s}$ and its derivatives}

Let us remember first the transport equation for $\chi$ :

$$
L(\chi)=-\chi^{2}-\kappa_{N N} \chi-R_{L L}
$$

where $R_{L L}$ has the form

$$
R_{L L}=L(v)+E .
$$

Due to Proposition 3.3, $v$ satisfies $|v| \lesssim|\partial g|$ and the error term $E$ has the property $|E| \lesssim|\partial g|^{2}+\left|\square_{g} g\right|$. Using this decomposition we can write the transport equation in the form:

$$
L\left(\chi-\frac{1}{s}+v\right)+2 \chi\left(\chi-\frac{1}{s}+v\right)=\left(\chi-\frac{1}{s}\right)^{2}+2 \chi\left(v-\frac{1}{2} \kappa_{N N}\right)-E
$$

Due to the bootstrap assumption $\left|\chi-\frac{1}{s}\right| \leq R_{0}$ :

$$
s^{2}\left|\chi-\frac{1}{s}+v\right| \rightarrow 0 \quad \text { as } \quad s \rightarrow 0
$$

We are now in a position to apply (77) with $\sigma=2$. Therefore we can infer that: 


$$
\sup _{S_{t, u}}\left|\chi-\frac{1}{s}+v\right| \lesssim \frac{4}{s^{2}} \int_{0}^{s} \rho^{2}\left|\left(\chi-\frac{1}{\rho}\right)^{2}+2 \chi\left(v-\frac{1}{2} \kappa_{N N}\right)-E\right| d \rho .
$$

Using again the above bootstrap assumption and the fact that $s R_{0} \ll 1$, together with the estimates for $v$ and $E$, obtained in Corollary 3.4, we conclude that:

$$
\sup _{S_{t, u}}\left|\chi-\frac{1}{s}\right| \leq 4 s R_{0}^{2}+8 s\left(R_{0}+\frac{1}{s}\right) \lambda^{-\bar{a}}+\lambda^{a-2} \leq \frac{R_{0}}{2}+\lambda^{-\bar{a}},
$$

which implies the desired estimate for $\sup _{S_{t, u}}\left|\chi-\frac{1}{s}\right|$.

Remark From now on, throughout the rest of this section, this argument will be considered implicit and so we will ignore in the deduction of further estimates the integral terms which are part of the bootstrap argument. For more details see section 5.7 in [7].

An immediate consequence of this result is the estimate for $\left\|L\left(\chi-\frac{1}{s}\right)\right\|_{L^{2}\left(S_{t, u}\right)}$. Writing once again the transport equation in the form

$$
L\left(\chi-\frac{1}{s}\right)=-\chi^{2}+\frac{1}{s^{2}}-\kappa_{N N} \chi-R_{L L}
$$

we obtain by taking the $L^{2}\left(S_{t, u}\right)$ norm:

$$
\left\|L\left(\chi-\frac{1}{s}\right)\right\|_{L^{2}\left(S_{t, u}\right)} \lesssim s^{\frac{1}{2}} \lambda^{-\bar{a}}\left(\lambda^{-\bar{a}}+\frac{2}{s}\right)+\lambda^{-\bar{a}-\frac{1-a}{2}+\epsilon} \lesssim \lambda^{-\bar{a}-\frac{1-a}{2}+\epsilon}+s^{-\frac{1}{2}} \lambda^{-\bar{a}}
$$

For the other two derivatives of $\chi-\frac{1}{s}$ we need the following

Lemma 4.2 The term $v$ and the error E satisfy

$$
\begin{aligned}
& \|\mathcal{D} v\|_{L^{2}\left(S_{t, u}\right)} \lesssim \lambda^{-\bar{a}-\frac{1-a}{2}+\epsilon}+s^{-\frac{1}{2}} \lambda^{-\bar{a}} \\
& \int_{0}^{s} \rho^{\frac{1}{2}}\|\mathcal{D} E\|_{L^{2}\left(S_{\rho+u, u}\right)} d \rho \lesssim \lambda^{2 a-2 \bar{a}-\frac{1}{2}+\epsilon}
\end{aligned}
$$

Proof: $v$ contains terms of the type $L^{\nu} g^{\alpha \beta} \partial_{\beta} g_{\alpha \nu}$, so its derivatives would depend on:

$$
\begin{aligned}
& \left|L^{\nu} \mathcal{D}\left(g^{\alpha \beta}\right) \partial_{\beta} g_{\alpha \nu}\right| \lesssim|\partial g|^{2} \\
& \left|L^{\nu} g^{\alpha \beta} \mathcal{D} \partial_{\beta} g_{\alpha \nu}\right| \lesssim\left|\partial^{2} g\right| \\
& \left|\mathcal{D}\left(L^{\nu}\right) g^{\alpha \beta} \partial_{\beta} g_{\alpha \nu}\right| \lesssim\left|\mathcal{D}\left(L^{\nu}\right)\right||\partial g|
\end{aligned}
$$

For the first two terms the estimates are immediately due to the bounds that we have for $\kappa$ and $R$ :

$$
\begin{aligned}
& \left\|L^{\nu} \mathcal{D}\left(g^{\alpha \beta}\right) \partial_{\beta} g_{\alpha \nu}\right\|_{L^{2}\left(S_{t, u}\right)} \lesssim s^{\frac{1}{2}} \lambda^{-2 \bar{a}} \\
& \left\|L^{\nu} g^{\alpha \beta} \mathcal{D} \partial_{\beta} g_{\alpha \nu}\right\|_{L^{2}\left(S_{t, u}\right)} \lesssim \lambda^{-\bar{a}-\frac{1-a}{2}+\epsilon}
\end{aligned}
$$

For the third term, we show how to obtain the estimate in the case when the derivative is $\not$, for $L$, respectively $\underline{L}$, the approach is similar. 


$$
\begin{aligned}
\not\left(L^{\nu}\right) & =\not\left(g^{\nu \mu}<L, \partial_{\mu}>\right)= \\
& =\not\left(g^{\nu \mu}\right) L_{\mu}+g^{\nu \mu}<\mathcal{D}_{A} L, \partial_{\mu}>+g^{\nu \mu}<L, \mathcal{D}_{A} \partial_{\mu}> \\
& =\not\left(g^{\nu \mu}\right) L_{\mu}+g^{\nu \mu} A^{\zeta} L_{\delta} \Gamma_{\zeta \mu}^{\delta}+g^{\nu \mu} A_{\mu} \chi-g^{\nu \mu} L_{\mu} \kappa_{A N}
\end{aligned}
$$

Using the assumptions and the estimates proved so far we conclude :

$$
\left|\mathcal{D}\left(L^{\nu}\right) g^{\alpha \beta} \partial_{\beta} g_{\alpha \nu}\right| \lesssim \lambda^{-\bar{a}}\left(\lambda^{-\bar{a}}+\frac{1}{s}\right)
$$

which yields

$$
\left\|\mathcal{D}\left(L^{\nu}\right) g^{\alpha \beta} \partial_{\beta} g_{\alpha \nu}\right\|_{L^{2}\left(S_{t, u}\right)} \lesssim s^{-\frac{1}{2}} \lambda^{-\bar{a}}
$$

Putting together (92) and (93) we obtain the estimate for $\|\mathcal{D} v\|_{L^{2}\left(S_{t, u}\right)}$. The error term $E$ has the expression

$$
\begin{aligned}
E & =-\frac{1}{2} L^{\mu} L^{\nu} \square_{g} g_{\mu \nu}+L^{\mu} L^{\nu} g^{\alpha \beta} g_{\gamma \delta}\left(\Gamma_{\mu \beta}^{\gamma} \Gamma_{\alpha \nu}^{\delta}-\Gamma_{\mu \nu}^{\gamma} \Gamma_{\alpha \beta}^{\delta}\right)- \\
& -L\left(L^{\nu}\right) g^{\alpha \beta} \partial_{\beta} g_{\alpha \nu}-L^{\nu} L\left(g^{\alpha \beta}\right) \partial_{\beta} g_{\alpha \nu},
\end{aligned}
$$

so for its derivatives we have to deal with the following types of terms:

$$
\begin{aligned}
& \left|\mathcal{D}\left(L^{\mu}\right) L^{\nu} \square_{g} g_{\mu \nu}\right| \lesssim \frac{1}{s}\left|\square_{g} g\right| \Longrightarrow \\
& \Longrightarrow \int_{0}^{s} \rho^{\frac{1}{2}}\left\|\frac{1}{\rho}\left|\square_{g} g\right|\right\|_{L^{2}\left(S_{\rho+u, u}\right)} d \rho \lesssim\left\|\square_{g} g\right\|_{L_{\left[0, t_{*}\right]}^{1} L_{x}^{\infty}} \lesssim \lambda^{a-2} \\
& \left|L^{\mu} L^{\nu} \mathcal{D} \square_{g} g_{\mu \nu}\right| \lesssim\left|\partial \square_{g} g\right| \Longrightarrow \\
& \Longrightarrow \int_{0}^{s} \rho^{\frac{1}{2}}\left\|\partial \square_{g} g \mid\right\|_{L^{2}\left(S_{\rho+u, u}\right)} d \rho \lesssim s\left\|\partial \square_{g} g\right\|_{L_{\left[0, t_{*}\right]}^{1} L_{x}^{\infty}} \lesssim s \lambda^{2 a-3} \\
& \left|\mathcal{D}\left(L^{\mu}\right) L^{\nu} g^{\alpha \beta} g_{\gamma \delta} \Gamma_{\mu \beta}^{\gamma} \Gamma_{\alpha \nu}^{\delta}\right| \lesssim \frac{1}{s}|\partial g|^{2} \Longrightarrow \\
& \Longrightarrow \int_{0}^{s} \rho^{\frac{1}{2}}\left\|\frac{1}{\rho}(\partial g)^{2}\right\|_{L^{2}\left(S_{\rho+u, u}\right)} d \rho \lesssim\|\partial g\|_{L_{\left[0, t_{*}\right]}^{2} L_{x}^{\infty}} \lesssim \lambda^{a-2} \\
& \left|L^{\mu} L^{\nu} \mathcal{D}\left(g^{\alpha \beta}\right) g_{\gamma \delta} \Gamma_{\mu \beta}^{\gamma} \Gamma_{\alpha \nu}^{\delta}\right| \lesssim|\partial g|^{3} \Longrightarrow \\
& \Longrightarrow \int_{0}^{s} \rho^{\frac{1}{2}}\left\|(\partial g)^{3}\right\|_{L^{2}\left(S_{\rho+u, u}\right)} d \rho \lesssim s \lambda^{a-2-\bar{a}} \\
& \left|L^{\mu} L^{\nu} g^{\alpha \beta} g_{\gamma \delta} \mathcal{D} \Gamma_{\mu \beta}^{\gamma} \Gamma_{\alpha \nu}^{\delta}\right| \lesssim|\partial g|\left|\partial^{2} g\right| \Longrightarrow \\
& \Longrightarrow \int_{0}^{s} \rho^{\frac{1}{2}}\left\||\partial g|\left|\partial^{2} g\right|\right\|_{L^{2}\left(S_{\rho+u, u}\right)} d \rho \lesssim s^{\frac{1}{2}} \lambda^{-\bar{a}-\frac{3(1-a)}{2}+\epsilon} \\
& \left|L\left(L^{\nu}\right) \mathcal{D}\left(g^{\alpha \beta}\right) \partial_{\beta} g_{\alpha \nu}\right| \lesssim\left|(\partial g)^{3}\right| \Longrightarrow \\
& \Longrightarrow \int_{0}^{s} \rho^{\frac{1}{2}}\left\|(\partial g)^{3}\right\|_{L^{2}\left(S_{\rho+u, u}\right)} d \rho \lesssim s \lambda^{a-2-\bar{a}} \\
& \left|L\left(L^{\nu}\right) g^{\alpha \beta} \mathcal{D}\left(\partial_{\beta} g_{\alpha \nu}\right)\right| \lesssim\left|\partial g \| \partial^{2} g\right| \Longrightarrow \\
& \Longrightarrow \int_{0}^{s} \rho^{\frac{1}{2}}\left\||\partial g|\left|\partial^{2} g\right|\right\|_{L^{2}\left(S_{\rho+u, u}\right)} d \rho \lesssim s^{\frac{1}{2}} \lambda^{-\bar{a}-\frac{3(1-a)}{2}+\epsilon} \\
& \Longrightarrow \\
& \Longrightarrow \\
& \Longrightarrow
\end{aligned}
$$




$$
\begin{aligned}
& \left|\mathcal{D} L\left(L^{\nu}\right) g^{\alpha \beta} \partial_{\beta} g_{\alpha \nu}\right| \lesssim|\partial g|\left(\left|\partial^{2} g\right|+|\partial g|^{2}+\frac{1}{s}|\partial g|+\left|\partial\left(\kappa_{N N}\right)\right|\right) \Longrightarrow \\
& \Longrightarrow \int_{0}^{s} \rho^{\frac{1}{2}}\left\||\partial g|\left(\left|\partial^{2} g\right|+|\partial g|^{2}+\frac{1}{s}|\partial g|+\left|\partial\left(\kappa_{N N}\right)\right|\right)\right\|_{L^{2}\left(S_{\rho+u, u}\right)} d \rho \lesssim \\
& \lesssim \lambda^{a-2}+s \lambda^{a-2-\bar{a}}+s^{\frac{1}{2}} \lambda^{-\bar{a}-\frac{3(1-a)}{2}+\epsilon}+s \lambda^{-2 \bar{a}}+s^{\frac{3}{2}} \lambda^{-2 \bar{a}-\frac{(1-a)}{2}+\epsilon}
\end{aligned}
$$

Using the bound $s \leq \lambda^{a}$, we obtain the desired result. Having obtained the estimates for $v$ and $E$, we can proceed to the investigation of the other two derivatives of $\chi-\frac{1}{s}$. The $\underline{L}$ derivative will be discussed later in connection with $\eta$. We deal now with the angular derivative $\not \nabla$. The estimate is obtained using the transport equation for this quantity. To simplify the notation we denote $\chi-\frac{1}{s}$ by $w$. Let us remember first the transport equation for this quantity:

$$
L(w+v)+2 \chi(w+v)=(w+v)^{2}+\frac{2}{s} v-v^{2}-\kappa_{N N} \chi-E .
$$

Taking the angular derivative of this expression we obtain:

$$
\begin{aligned}
A(L(w+v))+2 A(w)(w+v) & +2 \chi A(w+v)=2 A(w+v)(w+v)+ \\
& +A\left(\frac{2}{s} v-v^{2}-\kappa_{N N} \chi-E\right)
\end{aligned}
$$

Using the commutator $[L, A]=-\chi A$, we can write the previous equation in the form:

$$
L(\not \nabla(w+v))+3 \chi \not \nabla(w+v)=2 \not \nabla(v)(w+v)+\not\left(\frac{2}{s} v-v^{2}-\kappa_{N N} \chi-E\right)
$$

We will apply (78) with $\sigma=3$. Therefore, the only thing to be verified is that

$$
s^{\frac{5}{2}}\|\not \nabla(w+v)\|_{L^{2}\left(S_{t, u}\right)} \longrightarrow 0 \quad \text { as } \quad s \longrightarrow 0
$$

But this is true, because due to the bootstrap assumption (71) and the estimates for the derivatives of $v$ we have:

$$
\begin{aligned}
s^{\frac{5}{2}}\|\not \nabla(w)\|_{L^{2}\left(S_{t, u}\right)} & \leq R_{0} s^{2} \\
s^{\frac{5}{2}}\|\not \nabla(v)\|_{L^{2}\left(S_{t, u}\right)} & \lesssim s^{\frac{5}{2}} \lambda^{-\bar{a}-\frac{1-a}{2}+\epsilon}+s^{2} \lambda^{-\bar{a}}
\end{aligned}
$$

So applying the above mentioned estimate, we infer that:

$$
s^{\frac{5}{2}}\|\not \nabla(w+v)\|_{L^{2}\left(S_{t, u}\right)} \lesssim \int_{0}^{s} \rho^{\frac{5}{2}}\left\|2 \not \nabla(v)(w+v)+\not \nabla\left(\frac{2}{s} v-v^{2}-\kappa_{N N} \chi-E\right)\right\|_{L^{2}\left(S_{\rho+u, u}\right)} d \rho
$$

Using the estimates for $w=\chi-\frac{1}{s}, v$ and derivatives of $v$ and $E$, we conclude that:

$$
\|\not(w)\|_{L^{2}\left(S_{t, u}\right)} \lesssim \lambda^{-\bar{a}-\frac{1-a}{2}+\epsilon}+s^{-\frac{1}{2}} \lambda^{-\bar{a}}
$$

Finally we investigate $\underline{L}\left(\chi-\frac{1}{s}\right)$. We take advantage of the elliptic equation (65)

$$
\underline{L}(\chi)-\chi^{2}-\left(\kappa_{N N}+2 \kappa_{A A}\right) \chi=2 \not \nabla(\eta)+2 \eta^{2}+R_{A L \underline{L A}}
$$


Let us denote $\underline{L}(\chi)-\chi^{2}$ by $\theta$ and the whole left side of the above equality by $\Theta$. First we can write:

$L(\theta)+2 \chi \theta=2(\underline{\eta}-\eta) \not(\chi)+3 \kappa_{N N} \chi^{2}+\left(\left(\kappa_{N N}\right)^{2}+2 R_{L L}-\underline{L}\left(\kappa_{N N}\right)\right) \chi+\kappa_{N N} R_{L L}-\underline{L}\left(R_{L L}\right)$

This equation implies then

$$
\begin{aligned}
L(\Theta)+2 \chi \Theta & =2(\underline{\eta}-\eta) \not(\chi)+2\left(\kappa_{N N}-\kappa_{A A}\right) \chi^{2}+2\left(-T\left(\kappa_{N N}\right)-L\left(\kappa_{A A}\right)+\right. \\
& \left.+\left(\kappa_{N N}\right)^{2}+\kappa_{N N} \kappa_{A A}+R_{L L}\right) \chi+2\left(\kappa_{N N}+\kappa_{A A}\right) R_{L L}-\underline{L}\left(R_{L L}\right)
\end{aligned}
$$

We will denote by $F$ the right hand side of the above equation. Taking advantage of the special structure for $\underline{L}\left(R_{L L}\right)$ :

$$
\begin{aligned}
-\underline{L}\left(R_{L L}\right) & =-\underline{L}(L(v)+E)=-L(\underline{L}(v))-\underline{L}(E)+[L, \underline{L}](v)= \\
& =-L(\underline{L}(v))-\underline{L}(E)+2(\underline{\eta}-\eta) \not \nabla(v)+\kappa_{N N}(\underline{L}(v)-L(v))
\end{aligned}
$$

we can conclude that

$L(\Theta+\underline{L}(v))+2 \chi(\Theta+\underline{L}(v))=F^{\prime}-\underline{L}(E)+2(\underline{\eta}-\eta) \not \nabla(v)+\left(\kappa_{N N}+2 \chi\right) \underline{L}(v)-\kappa_{N N} L(v)=W$.

where we denoted by $F^{\prime}$, all the terms in $F$ but the last one. We rely once again on (78) with $\sigma=2$. The bootstrap assumption (71) and the estimate (91) imply

$$
s^{\frac{3}{2}}\|\Theta+\underline{L}(v)\|_{L^{2}\left(S_{t, u}\right)} \longrightarrow 0 \quad \text { as } \quad s \longrightarrow 0
$$

Applying the result mentioned above, we deduce:

$$
s^{\frac{3}{2}}\|\Theta+\underline{L}(v)\|_{L^{2}\left(S_{t, u}\right)} \lesssim \int_{0}^{s} \rho^{\frac{3}{2}}\|W\|_{L^{2}\left(S_{\rho+u, u}\right)} d \rho
$$

Evaluating all the terms in $F^{\prime}$ we obtain:

$$
\begin{aligned}
& \frac{1}{s^{\frac{3}{2}}} \int_{0}^{s} \rho^{\frac{3}{2}}\|(\underline{\eta}-\eta) \not(\chi)\|_{L^{2}\left(S_{\rho+u, u}\right)} d \rho \lesssim \lambda^{-\bar{a}-\frac{1-a}{2}+\epsilon}+s^{-\frac{1}{2}} \lambda^{-\bar{a}} \\
& \frac{1}{s^{\frac{3}{2}}} \int_{0}^{s} \rho^{\frac{3}{2}}\left\|\kappa(\chi)^{2}\right\|_{L^{2}\left(S_{\rho+u, u}\right)} d \rho \lesssim s^{-\frac{1}{2}} \lambda^{-\bar{a}} \\
& \frac{1}{s^{\frac{3}{2}}} \int_{0}^{s} \rho^{\frac{3}{2}}\left\|\left(\partial \kappa+(\kappa)^{2}\right) \chi\right\|_{L^{2}\left(S_{\rho+u, u}\right)} d \rho \lesssim \lambda^{-\bar{a}-\frac{1-a}{2}+\epsilon}+s^{-\frac{1}{2}} \lambda^{-\bar{a}} \\
& \frac{1}{s^{\frac{3}{2}}} \int_{0}^{s} \rho^{\frac{3}{2}}\|(|\kappa|+|\chi|) \mid R\|_{L^{2}\left(S_{\rho+u, u}\right)} d \rho \lesssim \lambda^{-\bar{a}-\frac{1-a}{2}+\epsilon}
\end{aligned}
$$

For all the other terms in $W$, we use the estimates (91) to argue that:

$$
\begin{aligned}
& \frac{1}{s^{\frac{3}{2}}} \int_{0}^{s} \rho^{\frac{3}{2}}\|\underline{L}(E)\|_{L^{2}\left(S_{\rho+u, u}\right)} d \rho \lesssim s^{-\frac{1}{2}} \lambda^{-2 \bar{a}+2 a-\frac{1}{2}+\epsilon} \\
& \frac{1}{s^{\frac{3}{2}}} \int_{0}^{s} \rho^{\frac{3}{2}}\|(\underline{\eta}-\eta) \not \nabla(v)\|_{L^{2}\left(S_{\rho+u, u}\right)} d \rho \lesssim \lambda^{-\bar{a}-\frac{1-a}{2}+\epsilon}+s^{-\frac{1}{2}} \lambda^{-\bar{a}} \\
& \frac{1}{s^{\frac{3}{2}}} \int_{0}^{s} \rho^{\frac{3}{2}}\|(|\kappa|+|\chi|)|\partial v|\|_{L^{2}\left(S_{\rho+u, u}\right)} d \rho \lesssim \lambda^{-\bar{a}-\frac{1-a}{2}+\epsilon}+s^{-\frac{1}{2}} \lambda^{-\bar{a}}
\end{aligned}
$$


This allows to conclude:

$$
\|\Theta+\underline{L}(v)\|_{L^{2}\left(S_{t, u}\right)} \lesssim \lambda^{-\bar{a}-\frac{1-a}{2}+\epsilon}+s^{-\frac{1}{2}} \lambda^{-\bar{a}}
$$

which in turn implies

$$
\|\Theta\|_{L^{2}\left(S_{t, u}\right)} \lesssim \lambda^{-\bar{a}-\frac{1-a}{2}+\epsilon}+s^{-\frac{1}{2}} \lambda^{-\bar{a}}
$$

based on the estimates that we have for $v$. We will deal now with $b$, because the estimate for $|b-1|$ will help us finish the proof for $\underline{L}\left(\chi-\frac{1}{s}\right)$. The transport equation for $b$ is

$$
L(b)=-b \kappa_{N N}
$$

We can apply (77) with $\sigma=0$, because the initial values (69) imply

$$
|b-1| \longrightarrow 0 \quad \text { as } \quad s \longrightarrow 0
$$

Therefore, we obtain:

$$
|b-1| \lesssim \int_{0}^{s}|\kappa| d \rho \lesssim \min \left\{s \lambda^{-\bar{a}}, \lambda^{-(1-a)}\right\}
$$

Rewriting $\Theta$ as

$$
\Theta=\underline{L}\left(\chi-\frac{1}{s}\right)+2 \frac{b^{-1}-1}{s^{2}}+\left(\frac{1}{s^{2}}-\chi^{2}\right)-\left(\kappa_{N N}+2 \kappa_{A A}\right) \chi
$$

we can infer that

$$
\begin{aligned}
\left\|\underline{L}\left(\chi-\frac{1}{s}\right)\right\|_{L^{2}\left(S_{t, u}\right)} & \lesssim\|\Theta\|_{L^{2}\left(S_{t, u}\right)}+\frac{1}{s^{2}}\|b-1\|_{L^{2}\left(S_{t, u}\right)}+\left\|\frac{1}{s^{2}}-\chi^{2}\right\|_{L^{2}\left(S_{t, u}\right)}+\|\kappa \chi\|_{L^{2}\left(S_{t, u}\right)} \\
& \lesssim \lambda^{-\bar{a}-\frac{1-a}{2}+\epsilon}+s^{-\frac{1}{2}} \lambda^{-\bar{a}}
\end{aligned}
$$

which concludes our discussion.

\subsection{Proof of the estimates for $\eta$ and its derivatives}

We remember what we have denoted by $\Theta$

$$
\Theta=2 \not \nabla(\eta)+2 \eta^{2}+R_{A L \underline{L A}}
$$

We can write then

$$
\not(\eta)+\eta^{2}=H
$$

where $H=\frac{1}{2}\left(\Theta-R_{A L \underline{L A}}\right)$ satisfies

$$
\|H\|_{L^{2}\left(S_{t, u}\right)} \lesssim \lambda^{-\bar{a}-\frac{1-a}{2}+\epsilon}+s^{-\frac{1}{2}} \lambda^{-\bar{a}}
$$


Using (67), we can conclude that

$$
\begin{aligned}
& \|\not(\eta)\|_{L^{2}\left(S_{t, u}\right)} \lesssim\|H\|_{L^{2}\left(S_{t, u}\right)} \lesssim \lambda^{-\bar{a}-\frac{1-a}{2}+\epsilon}+s^{-\frac{1}{2}} \lambda^{-\bar{a}} \\
& \|\eta\|_{L^{4}\left(S_{t, u}\right)}^{2} \lesssim\|H\|_{L^{2}\left(S_{t, u}\right)} \lesssim \lambda^{-\bar{a}-\frac{1-a}{2}+\epsilon}+s^{-\frac{1}{2}} \lambda^{-\bar{a}}
\end{aligned}
$$

We use the transport equation satisfied by $\eta$

$$
L(\eta)+\chi \eta=-\chi \kappa_{A N}+\frac{1}{2} R_{A L L \underline{L}}
$$

with the initial conditions

$$
\begin{aligned}
& s \eta \longrightarrow 0 \\
& s^{\frac{1}{2}}\|\eta\|_{L^{2}\left(S_{t, u}\right)} \longrightarrow 0
\end{aligned}
$$

as $s \longrightarrow 0$, satisfied due to the assumption (70). Applying (80) with $\sigma=1$, we conclude:

$$
\begin{aligned}
& \sup _{S_{t, u}}|\eta| \lesssim \sup _{\rho \leq s} \rho(|\kappa||\chi|+|R|) \lesssim s \lambda^{-\bar{a}-(1-a)}+\lambda^{-\bar{a}} \\
& \|\eta\|_{L^{2}\left(S_{t, u}\right)} \lesssim \frac{1}{s^{\frac{1}{2}}} \int_{0}^{s} \rho^{\frac{1}{2}}\||\kappa||\chi|+|R|\|_{L^{2}\left(S_{\rho+u, u}\right)} d \rho \lesssim s \lambda^{-\bar{a}-\frac{1-a}{2}+\epsilon}+s^{\frac{1}{2}} \lambda^{-\bar{a}}
\end{aligned}
$$

At this point we take advantage of the Sobolev inequality (73) to infer that:

$$
\sup _{S_{t, u}}|\eta| \lesssim s^{\frac{1}{2}}\|\not \nabla(\eta)\|_{L^{2}\left(S_{t, u}\right)}+s^{-\frac{1}{2}}\|\eta\|_{L^{2}\left(S_{t, u}\right)} \lesssim s^{\frac{1}{2}} \lambda^{-\bar{a}-\frac{1-a}{2}+\epsilon}+\lambda^{-\bar{a}}
$$

Due to this result and the transport equation for $\eta$ we obtain that $L(\eta)$ satisfies the same estimate as $\not(\eta)$. Here we remark that these estimates for $\eta$ imply the desired inequalities for the $\underline{L}$ derivatives of the second fundamental form $\kappa$. As in [17], for the case $n=3$, we do not have a good estimate for $\underline{L}(\eta)$. This finishes the discussion concerning $\eta$ and its derivatives.

\subsection{Proof of the estimates for $b$ and its derivatives}

We notice that already in the course of the proof for $\chi-\frac{1}{s}$ we obtained

$$
|b-1| \lesssim \int_{0}^{s}|\kappa| d \rho \lesssim \min \left\{s \lambda^{-\bar{a}}, \lambda^{-(1-a)}\right\}
$$

Due to the transport equation $L(b)=-b \kappa_{N N}$ we infer immediately that

$$
\begin{aligned}
& \sup _{S_{t, u}}|L(b)| \lesssim \lambda^{-\bar{a}} \\
& \|L(b)\|_{L^{2}\left(S_{t, u}\right)} \lesssim s^{\frac{1}{2}} \lambda^{-\bar{a}}
\end{aligned}
$$

The formula $\not{\nabla}(b)=b\left(\eta-\kappa_{A N}\right)$, together with the inequalities satisfied by $\eta$ and $\kappa$ : 


$$
\begin{aligned}
& \sup _{S_{t, u}}|\kappa| \lesssim \lambda^{-\bar{a}} \\
& \sup _{S_{t, u}}|\eta| \lesssim s^{\frac{1}{2}} \lambda^{-\bar{a}-\frac{1-a}{2}+\epsilon}+\lambda^{-\bar{a}},
\end{aligned}
$$

provide us immediately with the estimates

$$
\begin{aligned}
& \sup _{S_{t, u}}|\not \nabla(b)| \lesssim s^{\frac{1}{2}} \lambda^{-\bar{a}-\frac{1-a}{2}+\epsilon}+\lambda^{-\bar{a}} \\
& \|\not(b)\|_{L^{2}\left(S_{t, u}\right)} \lesssim s \lambda^{-\bar{a}-\frac{1-a}{2}+\epsilon}+s^{\frac{1}{2}} \lambda^{-\bar{a}}
\end{aligned}
$$

For $\underline{L}(b)$ we write its transport equation

$$
L(\underline{L}(b))=\underline{L}(L(b))+[L, \underline{L}](b)=b\left(\left(\kappa_{N N}\right)^{2}+2\left(\kappa_{A N}\right)^{2}-\underline{L}\left(\kappa_{N N}\right)-2 \eta^{2}\right)
$$

Due to the initial values (69), we have

$$
\begin{aligned}
& \underline{L}(b) \longrightarrow 0 \\
& s^{-\frac{1}{2}}\|\underline{L}(b)\|_{L^{2}\left(S_{t, u}\right)} \longrightarrow 0
\end{aligned}
$$

as $s \longrightarrow 0$. We apply (77) and (78) with $\sigma=0$ to deduce that:

$$
\begin{aligned}
& \sup _{S_{t, u}}|\underline{L}(b)| \lesssim s \lambda^{-\bar{a}-(1-a)} \\
& \|\underline{L}(b)\|_{L^{2}\left(S_{t, u}\right)} \lesssim s \lambda^{-\bar{a}-\frac{1-a}{2}+\epsilon}
\end{aligned}
$$

This concludes the proof of the asymptotic estimates.

\section{The reduction of Theorem 2.5 to conformal energy estimates}

We recall first, that according to Theorem 2.5, the estimate to be proved is:

$$
\|P \partial \phi(t)\|_{L_{x}^{\infty}} \lesssim \frac{1}{\left(1+\left|t-t_{0}\right|\right)^{\frac{1}{2}-\epsilon}} \sum_{k=1}^{m}\left\|\partial^{k} \phi\left[t_{0}\right]\right\|_{L_{x}^{2}}
$$

for a sufficiently large integer $m$, where $\phi$ solves the equation $\square_{g} \phi=0$, the metric $g$ satisfies (27)-(32), $P$ is the operator of projection on the set $\left\{\xi: \frac{1}{2} \leq|\xi| \leq 2\right\}$ in Fourier space and the initial data $\phi\left[t_{0}\right]$ is supported in the ball $B_{\frac{1}{2}}(0)$. The argument here follows to some extent the same lines as the one in Section 7, [8]. For the interior region, due to the fact that $P$ is an operator acting on the scale of size 1 and $1-\omega$ is a cut-off function with the scale of size $t \geq 1$, we can basically write

$$
P \partial \phi \cdot(1-\omega) \approx P(\partial \phi \cdot(1-\omega))
$$


Therefore, using Bernstein inequality and the fact that $|\partial \omega| \lesssim t^{-1}$, we have the following chain of estimates:

$$
\begin{aligned}
\|P \partial \phi \cdot(1-\omega)\|_{L^{\infty}\left(\Sigma_{t}\right)} & \lesssim \| \partial P\left(\partial \phi \cdot(1-\omega) \|_{L^{2}\left(\Sigma_{t}\right)}\right. \\
& \lesssim\left\|\partial^{2} \phi \cdot(1-\omega)\right\|_{L^{2}\left(\Sigma_{t}\right)}+\frac{1}{t}\|\partial \phi \cdot(1-\omega)\|_{L^{2}\left(\Sigma_{t}\right)} \\
& \lesssim \frac{1}{t} E^{\frac{1}{2}}(\partial \phi)(t)
\end{aligned}
$$

Remark In the interior region the decay is $t^{-1}$, better than the overall decay estimate of $t^{-\frac{1}{2}}$. This consideration will turn out to be crucial in further steps of the proof.

For the exterior region, let us first remark that, due to $\frac{t}{2} \lesssim s \lesssim t$, we can consider $s \approx t$. Using the Sobolev inequality (73), we can infer that

$$
\|P \partial \phi\|_{L^{\infty}\left(S_{t, u}\right)} \lesssim t^{\frac{1}{2}}\|\not \nabla P \partial \phi\|_{L^{2}\left(S_{t, u}\right)}+\frac{1}{t^{\frac{1}{2}}}\|P \partial \phi\|_{L^{2}\left(S_{t, u}\right)}
$$

For the second term, applying the trace estimate (75) together with the fact that $t \geq 1$, we conclude that:

$$
\begin{aligned}
\frac{1}{t^{\frac{1}{2}}}\|P \partial \phi\|_{L^{2}\left(S_{t, u}\right)} & \lesssim \frac{1}{t^{\frac{1}{2}}}\|N P \partial \phi\|_{L^{2}\left(\Sigma_{t, e x t}\right)}^{\frac{1}{2}} \cdot\|P \partial \phi\|_{L^{2}\left(\Sigma_{t, e x t}\right)}^{\frac{1}{2}}+\frac{1}{t}\|P \partial \phi\|_{L^{2}\left(\Sigma_{t, e x t}\right)} \lesssim \\
& \lesssim \frac{1}{t^{\frac{1}{2}}} E^{\frac{1}{2}}(\partial \phi)(t)
\end{aligned}
$$

In the case of the first term in (108), again due to (75), it follows that:

$$
t^{\frac{1}{2}}\|\not \nabla P \partial \phi\|_{L^{2}\left(S_{t, u}\right)} \lesssim t^{\frac{1}{2}}\|N \not \nabla P \partial \phi\|_{L^{2}\left(\Sigma_{t, e x t}\right)}^{\frac{1}{2}} \cdot\|\not \nabla P \partial \phi\|_{L^{2}\left(\Sigma_{t, e x t}\right)}^{\frac{1}{2}}+\|\not \nabla P \partial \phi\|_{L^{2}\left(\Sigma_{t, e x t}\right)}
$$

For $\|N \not P P \partial \phi\|_{L^{2}\left(\Sigma_{t, e x t}\right)}^{\frac{1}{2}}$ we use the trivial estimate

$$
\|N \not \nabla P \partial \phi\|_{L^{2}\left(\Sigma_{t, e x t}\right)}^{\frac{1}{2}} \lesssim\|\partial \phi\|_{L^{2}\left(\Sigma_{t, e x t}\right)}^{\frac{1}{2}} \lesssim E^{\frac{1}{4}}(\partial \phi)(t),
$$

while for $\|\not \nabla P \partial \phi\|_{L^{2}\left(\Sigma_{t, e x t}\right)}$ it will be enough to prove that it decays like $\frac{1}{t}$, or more precisely:

$$
\|\not P P \partial \phi\|_{L^{2}\left(\Sigma_{t, e x t}\right)} \lesssim \frac{1}{t} E^{\frac{1}{2}}(\partial \phi)(t) .
$$

Commuting $P$ with $\not$ directly as in Lemma 7.1, [8], together with the null-frame equations contained in Theorem 3.5, we obtain the following chain of estimates:

$$
\begin{aligned}
& \|\not P P \partial \phi\|_{L^{2}\left(\Sigma_{t, e x t}\right)} \lesssim \sup _{\alpha, \beta}\left\|\partial_{\alpha} e_{A}^{\beta}\right\|_{L^{\infty}\left(\Sigma_{t, e x t}\right)} \cdot\|\partial \phi\|_{L^{2}\left(\Sigma_{t}\right)}+\|P \not \nabla \partial \phi\|_{L^{2}\left(\Sigma_{t, e x t}\right)} \lesssim \\
& \lesssim \max \left\{\|\chi\|_{L^{\infty}\left(\Sigma_{t, e x t}\right)},\|\eta\|_{L^{\infty}\left(\Sigma_{t, e x t}\right)},\|\kappa\|_{L^{\infty}\left(\Sigma_{t, e x t}\right)}\right\} \cdot\|\partial \phi\|_{L^{2}\left(\Sigma_{t}\right)}+\|\not \nabla \partial \phi\|_{L^{2}\left(\Sigma_{t, e x t}\right)} \\
& \lesssim\left(\frac{1}{t}+\lambda^{-\bar{a}}+t^{\frac{1}{2}} \lambda^{-\bar{a}-\frac{1-a}{2}+\epsilon}\right) E^{\frac{1}{2}}(\partial \phi)(t)+\frac{1}{t} E^{\frac{1}{2}}(\partial \phi)(t) \lesssim \frac{1}{t} E^{\frac{1}{2}}(\partial \phi)(t) .
\end{aligned}
$$


The inequality used here:

$$
t^{\frac{1}{2}} \lambda^{-\bar{a}-\frac{1-a}{2}+\epsilon} \lesssim \frac{1}{t}
$$

is the one that gives the range $a<\sqrt{22}-4$. Putting together (108)-(112), we infer that:

$$
\|P \partial \phi\|_{L^{\infty}\left(S_{t, u}\right)} \lesssim t^{-\frac{1}{2}} E^{\frac{1}{2}}(\partial \phi)(t)
$$

Thus, due to (107) and (115), Theorem 2.5 will be an immediate consequence of the following:

Theorem 5.1 If $\phi$ is a solution of the linear wave equation

$$
\begin{aligned}
& \square_{g} \phi=0, \\
& \left.\phi\right|_{t=0}=\phi_{0},\left.\quad \partial_{t} \phi\right|_{t=0}=\phi_{1}
\end{aligned}
$$

with the metric $g$ verifying the condition (27)-(32), $a<\sqrt{22}-4$ and the initial data $\phi\left[t_{0}\right]$ supported in the ball $B_{\frac{1}{2}}(0)$, then:

$$
\sup _{\tau \in\left[t_{0}, t_{*}\right]} E(\partial \phi)(\tau) \lesssim E(\partial \phi)\left(t_{0}\right)
$$

\section{Outline of the proof of Theorem 5.1}

In the case of dimension $n=3$, Klainerman and Rodnianski proved the corresponding decay estimate using only the Morawetz vectorfield $K$. They relied on the following generalized energy estimate:

$$
\int_{\Sigma_{t}} Q\left(K, \partial_{t}\right)(\phi) \gtrsim E(\phi)(t)
$$

where

$$
Q\left(K, \partial_{t}\right)(\phi)=\frac{1}{4}\left(\underline{u}^{2}|L \phi|^{2}+u^{2}|\underline{L} \phi|^{2}+\left(\underline{u}^{2}+u^{2}\right)|A \phi|^{2}\right)+(n-1) t \phi \partial_{t} \phi-\frac{n-1}{2} \phi^{2} .
$$

As it was first remarked by Klainerman in [5], this inequality is no longer true in the case of dimension $n=2$. It survives however in the form:

$$
\int_{\Sigma_{t}} Q\left(K, \partial_{t}\right)(\phi)+\|\phi\|_{L^{2}\left(\Sigma_{t}\right)}^{2} \gtrsim E(\phi)(t)
$$

Due to the fact that we do not control $\|\phi\|_{L^{2}\left(\Sigma_{t}\right)}$, we will have to use (119) with $\phi \rightarrow \partial_{t} \phi$, the quantity $\|\partial \phi\|_{L^{2}\left(\Sigma_{t}\right)}$ being bounded through the classical energy estimate. This modification will help us recover the desired estimate (117) only in the interior region. The novelty of our argument comes in the deduction of the required estimate in the exterior region. We will 
use two other vectorfields, $S$ and $\Omega$. We will commute them with the D'Alembertian $\square_{g}$, according to the formula:

$$
\square_{g}(X \phi)-X\left(\square_{g} \phi\right)={ }^{X} \pi^{\alpha \beta} \cdot \mathcal{D}_{\alpha} \mathcal{D}_{\beta} \phi+\mathcal{D}^{\alpha X} \pi_{\alpha \lambda} \cdot \mathcal{D}^{\lambda} \phi-\frac{1}{2} \mathcal{D}^{\lambda} \operatorname{tr}^{X} \pi \cdot \mathcal{D}_{\lambda} \phi=Z,
$$

where

$$
{ }^{X} \pi_{\alpha \beta}=\mathcal{D}_{\alpha} X_{\beta}+\mathcal{D}_{\beta} X_{\alpha}
$$

is the deformation tensor of the vectorfield $X$ relative to the metric $g$. For the equation $\square_{g}(X \phi)=Z$ we will use the classical energy estimate (see also (125)):

$$
\|\partial X \phi(t)\|_{L^{2}\left(\Sigma_{t}\right)} \lesssim\left(\left\|\partial X \phi\left(t_{0}\right)\right\|_{L^{2}\left(\Sigma_{t_{0}}\right)}+\int_{t_{0}}^{t}\|Z(\tau, \cdot)\|_{L^{2}\left(\Sigma_{\tau}\right)} d \tau\right) \cdot e^{C \int_{t_{0}}^{t}\left\|\partial_{t} g(\tau, \cdot)\right\|_{L^{\infty}\left(\Sigma_{\tau}\right)} d \tau}
$$

As it can be seen from (117), the goal will be to prove that:

$$
\int_{t_{0}}^{t}\|Z(\tau, \cdot)\|_{L^{2}\left(\Sigma_{\tau}\right)} d \tau \lesssim \lambda^{-\epsilon} \sup _{\tau \in\left[t_{0}, t\right]} E^{\frac{1}{2}}(\partial \phi)(\tau) .
$$

We will express $Z$ in equation (120) relative to the null frame $\{L, \underline{L}, A\}$. Accordingly, the deformation tensor ${ }^{X} \pi$ appears in terms of the Ricci coefficients $\chi, \eta$, the lapse function $b$ and the second fundamental form $\kappa$. Therefore, in order to estimate $Z$ in (122), we will need the bounds provided by the Asymptotics Theorem 4.1 in section 4 . A typical term that appears in (122), which comes from the combination ${ }^{\Omega} \pi_{\underline{L L}} \mathcal{D}_{L} \mathcal{D}_{L} \phi$, is

$$
\begin{aligned}
\int_{t_{0}}^{t}\|\eta \cdot s \cdot L \partial \phi\|_{L^{2}\left(\Sigma_{\tau}\right)} d \tau & \lesssim \int_{t_{0}}^{t}\|\eta\|_{L^{\infty}\left(\Sigma_{\tau}\right)} \cdot\|s \cdot L \partial \phi\|_{L^{2}\left(\Sigma_{\tau}\right)} d \tau \lesssim \\
& \lesssim \int_{t_{0}}^{t}\|\eta\|_{L^{\infty}\left(\Sigma_{\tau}\right)} d \tau \sup _{\tau \in\left[t_{0}, t\right]} E^{\frac{1}{2}}(\partial \phi)(\tau)
\end{aligned}
$$

The estimate provided by Theorem 4.1 for $\eta$ is

$$
\|\eta\|_{L^{\infty}\left(\Sigma_{\tau}\right)} \lesssim \lambda^{a-\bar{a}-\frac{1}{2}+\epsilon}
$$

Hence, to obtain the estimate (122), we need the condition

$$
\int_{t_{0}}^{t} \lambda^{a-\bar{a}-\frac{1}{2}} d \tau \lesssim \lambda^{2 a-\bar{a}-\frac{1}{2}+\epsilon} \lesssim \lambda^{-\epsilon}
$$

which gives the range $a<\sqrt{22}-4$. The last section will gather the estimates obtained in the next two sections for the vectorfields $K, \Omega$ and respectively $S$, and so conclude the argument. 


\section{$7 \quad$ Energy estimates involving the Morawetz vectorfield $K$}

The central result of this section will be:

Theorem 7.1 Under the assumptions of Theorem 5.1:

$$
E\left(\partial_{t} \phi\right)(t) \lesssim E\left(\partial_{t} \phi\right)\left(t_{0}\right)+\lambda^{-\epsilon} \sup _{\tau \in\left[t_{0}, t_{*}\right]} E(\partial \phi)(\tau)
$$

In order to prove this theorem we start first with

Lemma 7.2 ([7]) If $w$ is a solution of the equation $\square_{g} w=f, Q_{\alpha \beta}$ is the energy momentum tensor associated to this equation

$$
Q_{\alpha \beta}=\partial_{\alpha} w \partial_{\beta} w-\frac{1}{2} g_{\alpha \beta}\left(g^{\mu \nu} \partial_{\mu} w \partial_{\nu} w\right),
$$

$X$ is a vectorfield with the deformation tensor

$$
{ }^{X} \pi_{\alpha \beta}=\mathcal{D}_{\alpha} X_{\beta}+\mathcal{D}_{\beta} X_{\alpha},
$$

$O$ is a scalar function, ${ }^{X} \tilde{\pi}={ }^{X} \pi-O g$ and

$$
\bar{Q}(X, Y)=Q(X, Y)+\frac{n-1}{4} O \cdot w \cdot Y w-\frac{n-1}{8} w^{2} Y(O),
$$

then the following identity holds:

$$
\begin{aligned}
\int_{\Sigma_{t}} \bar{Q}\left(X, \partial_{t}\right) & =\int_{\Sigma_{t_{0}}} \bar{Q}\left(X, \partial_{t}\right)+\frac{1}{2} \int_{\left[t_{0}, t\right] \times \mathbb{R}^{n}} Q^{\alpha \beta X} \tilde{\pi}_{\alpha \beta}-\frac{n-1}{8} \int_{\left[t_{0}, t\right] \times \mathbb{R}^{n}} w^{2} \square_{g} O+ \\
& +\int_{\left[t_{0}, t\right] \times \mathbb{R}^{n}}\left(X w+\frac{n-1}{4} O w\right) f .
\end{aligned}
$$

Proof Differentiating directly in the formula for $Q_{\alpha \beta}$, we obtain:

$$
\mathcal{D}^{\beta} Q_{\alpha \beta}=\mathcal{D}_{\alpha} w f \text {. }
$$

If we consider the 1 -form $P_{\alpha}=Q_{\alpha \beta} X^{\beta}$, previous equation leads to

$$
\mathcal{D}^{\alpha} P_{\alpha}=X w f+\frac{1}{2} Q_{\alpha \beta}{ }^{X} \pi^{\alpha \beta},
$$

which we can detail as:

$$
\begin{aligned}
\mathcal{D}^{\alpha} P_{\alpha} & =\frac{1}{2} X^{X} \tilde{\pi}^{\alpha \beta} Q_{\alpha \beta}+\left(X w+\frac{n-1}{4} O w\right) f-\frac{n-1}{8} w^{2} \square_{g} O- \\
& -\frac{n-1}{4} \mathcal{D}^{\mu}\left(O \cdot w \cdot \mathcal{D}_{\mu} w-\frac{1}{2} \mathcal{D}_{\mu} O \cdot w^{2}\right) .
\end{aligned}
$$


Denoting

$$
\bar{P}_{\alpha}=P_{\alpha}+\frac{n-1}{4} O \cdot w \cdot \mathcal{D}_{\alpha} w-\frac{n-1}{8} w^{2} \mathcal{D}_{\alpha} O
$$

it follows that:

$$
\mathcal{D}^{\alpha} \bar{P}_{\alpha}=\frac{1}{2} Q^{\alpha \beta X} \tilde{\pi}_{\alpha \beta}+\left(X w+\frac{n-1}{4} O w\right) f-\frac{n-1}{8} w^{2} \square_{g} O
$$

Integrating this equation over the domain $\left[t_{0}, t\right] \times \mathbb{R}^{n}$, we obtain (124).

Corollary 7.3 For $w$ a solution of the equation $\square_{g} w=f$, the following estimate holds:

$$
\|\partial w(t)\|_{L^{2}\left(\Sigma_{t}\right)} \lesssim\left(\left\|\partial w\left(t_{0}\right)\right\|_{L^{2}\left(\Sigma_{t_{0}}\right)}+\int_{t_{0}}^{t}\|f(\tau, \cdot)\|_{L^{2}\left(\Sigma_{\tau}\right)} d \tau\right) \cdot e^{C \int_{t_{0}}^{t}\left\|\partial_{t} g(\tau, \cdot)\right\|_{L^{\infty}\left(\Sigma_{\tau}\right)} d \tau}
$$

Proof Taking in the previous lemma, $X=\partial_{t}$ and $O=0$, we can rewrite (124) in the form:

$$
\begin{aligned}
\frac{1}{2} \int_{\Sigma_{t}}\left(\left|\partial_{t} w\right|^{2}\right. & \left.+g^{i j} \partial_{i} w \cdot \partial_{j} w\right)=\frac{1}{2} \int_{\Sigma_{t_{0}}}\left(\left|\partial_{t} w\right|^{2}+g^{i j} \partial_{i} w \cdot \partial_{j} w\right)+ \\
& +\frac{1}{2} \int_{\left[t_{0}, t\right] \times \mathbb{R}^{n}} Q^{\alpha \beta} \cdot \partial_{t} g_{\alpha \beta}+\int_{\left[t_{0}, t\right] \times \mathbb{R}^{n}} \partial_{t} w \cdot f
\end{aligned}
$$

Using the fact that the metric $g^{i j}$ is uniformly elliptic and bounded, we obtain

$$
\begin{aligned}
\|\partial w(t)\|_{L^{2}\left(\Sigma_{t}\right)}^{2} \lesssim\left\|\partial w\left(t_{0}\right)\right\|_{L^{2}\left(\Sigma_{t_{0}}\right)}^{2} & +\int_{t_{0}}^{t}\left(\left\|\partial_{t} g(\tau)\right\|_{L^{\infty}\left(\Sigma_{\tau}\right)} \cdot\|\partial w(\tau)\|_{L^{2}\left(\Sigma_{\tau}\right)}^{2}+\right. \\
& \left.+\|f(\tau)\|_{L^{2}\left(\Sigma_{\tau}\right)} \cdot\|\partial w(\tau)\|_{L^{2}\left(\Sigma_{\tau}\right)}\right) d \tau
\end{aligned}
$$

Applying Gronwall inequality to this equation concludes the proof.

Proposition 7.4 Under the assumptions of Theorem 5.1:

$$
\begin{aligned}
E\left(\partial_{t} \phi\right)(t) \lesssim E\left(\partial_{t} \phi\right)\left(t_{0}\right) & +\lambda^{-\epsilon} \sup _{\tau \in\left[t_{0}, t_{*}\right]} E(\partial \phi)(\tau)+ \\
& +\left|\int_{\left[t_{0}, t\right] \times \mathbb{R}^{2}} \frac{1}{2} Q^{\alpha \beta} \tilde{\pi}_{\alpha \beta}+\left(K \partial_{t} \phi+\tau \partial_{t} \phi\right) \square_{g}\left(\partial_{t} \phi\right)\right|
\end{aligned}
$$

Proof In the Lemma 7.2, we will choose $w=\partial_{t} \phi, n=2, X=K$ and $O=4 t$, this last choice being motivated by the fact that in Minkowski space $\operatorname{tr}^{K} \pi=4 t$ and that we are looking for an $O$ which would make ${ }^{K} \bar{\pi}$ small. (124) then becomes:

$$
\begin{aligned}
\int_{\Sigma_{t}} \bar{Q}\left(K, \partial_{t}\right) & =\int_{\Sigma_{t_{0}}} \bar{Q}\left(K, \partial_{t}\right)+\frac{1}{2} \int_{\left[t_{0}, t\right] \times \mathbb{R}^{2}} Q^{\alpha \beta K} \tilde{\pi}_{\alpha \beta}-\frac{1}{2} \int_{\left[t_{0}, t\right] \times \mathbb{R}^{2}}\left(\partial_{t} \phi\right)^{2} \square_{g}(\tau)+ \\
& +\int_{\left[t_{0}, t\right] \times \mathbb{R}^{2}}\left(K \partial_{t} \phi+\tau \partial_{t} \phi\right) \square_{g}\left(\partial_{t} \phi\right)
\end{aligned}
$$

We take apart the term $\bar{Q}\left(K, \partial_{t}\right)$ and write it in the more convenient form: 


$$
\begin{aligned}
\bar{Q}\left(K, \partial_{t}\right) & =Q\left(K, \partial_{t}\right)+t \cdot \partial_{t} \phi \cdot \partial_{t t} \phi-\frac{1}{2}\left(\partial_{t} \phi\right)^{2} \\
& =\frac{1}{4} \underline{u}^{2}\left|L \partial_{t} \phi\right|^{2}+\frac{1}{4} u^{2}\left|\underline{L} \partial_{t} \phi\right|^{2}+\frac{1}{4}\left(\underline{u}^{2}+u^{2}\right)\left|A \partial_{t} \phi\right|^{2}+t \cdot \partial_{t} \phi \cdot \partial_{t t} \phi- \\
& -\frac{1}{2}\left(\partial_{t} \phi\right)^{2}
\end{aligned}
$$

We will be interested in the particular term:

$$
\int_{\Sigma_{t}} t \cdot \partial_{t} \phi \cdot \partial_{t t} \phi=\int_{\Sigma_{t}} \partial_{t} \phi \cdot\left(S \partial_{t} \phi-(t-u) N \partial_{t} \phi\right) .
$$

We separate and integrate by parts the term involving $N \partial_{t} \phi$ :

$$
\begin{aligned}
& -\int_{\Sigma_{t}}(t-u) N \partial_{t} \phi \cdot \partial_{t} \phi=-\int_{\Sigma_{t}} \frac{1}{2}(t-u) N\left(\left(\partial_{t} \phi\right)^{2}\right)= \\
& =\int_{\Sigma_{t}} \frac{1}{2}\left(N(t-u)+\left(\chi+\kappa_{A A}\right)(t-u)\right)\left(\partial_{t} \phi\right)^{2}=\int_{\Sigma_{t}} \frac{1}{2}\left(b^{-1}+s \chi+s \kappa_{A A}\right)\left(\partial_{t} \phi\right)^{2}
\end{aligned}
$$

Using the asymptotic estimates:

$$
\begin{aligned}
& |b-1| \lesssim \lambda^{-\epsilon} \\
& \left|\chi-\frac{1}{s}\right|+|\kappa| \lesssim \lambda^{-\bar{a}},
\end{aligned}
$$

we conclude that:

$$
-\int_{\Sigma_{t}}(t-u) N \partial_{t} \phi \cdot \partial_{t} \phi=\int_{\Sigma_{t}}\left(1+O\left(\lambda^{-\epsilon}\right)\right)\left(\partial_{t} \phi\right)^{2}
$$

Hence

$$
\begin{aligned}
\int_{\Sigma_{t}} \bar{Q}\left(K, \partial_{t}\right)=\int_{\Sigma_{t}}\left(\frac{1}{4} \underline{u}^{2}\left|L \partial_{t} \phi\right|^{2}\right. & +\frac{1}{4} u^{2}\left|\underline{L} \partial_{t} \phi\right|^{2}+\frac{1}{4}\left(\underline{u}^{2}+u^{2}\right)\left|A \partial_{t} \phi\right|^{2}+ \\
& \left.+S \partial_{t} \phi \cdot \partial_{t} \phi+\left(\frac{1}{2}+O\left(\lambda^{-\epsilon}\right)\right)\left(\partial_{t} \phi\right)^{2}\right)
\end{aligned}
$$

Using the expression for $S$, it follows naturally that:

$$
\int_{\Sigma_{t}} \underline{u}^{2}\left|L \partial_{t} \phi\right|^{2}+u^{2}\left|\underline{L} \partial_{t} \phi\right|^{2}+\left(\underline{u}^{2}+u^{2}\right)\left|A \partial_{t} \phi\right|^{2} \lesssim \int_{\Sigma_{t}} \bar{Q}\left(K, \partial_{t}\right)+\left(\partial_{t} \phi\right)^{2},
$$

which of course implies

$$
E\left(\partial_{t} \phi\right)(t) \lesssim \int_{\Sigma_{t}} \bar{Q}\left(K, \partial_{t}\right)+\left\|\partial_{t} \phi\right\|_{L^{2}\left(\Sigma_{t}\right)}^{2}
$$

Applying the energy estimate (125), for our reduced linear problem $\square_{g} \phi=0$, we can infer that:

$$
\|\partial \phi(t)\|_{L^{2}\left(\Sigma_{t}\right)} \lesssim\left\|\partial \phi\left(t_{0}\right)\right\|_{L^{2}\left(\Sigma_{t_{0}}\right)} \cdot e^{C \int_{t_{0}}^{t}\left\|\partial_{t} g(\tau, \cdot)\right\|_{L^{\infty}\left(\Sigma_{\tau}\right)} d \tau}
$$


which due to

$$
\|\partial g\|_{L_{t}^{1} L_{x}^{\infty}} \lesssim \lambda^{-(1-a)}
$$

yields

$$
\|\partial \phi(t)\|_{L^{2}\left(\Sigma_{t}\right)} \lesssim\left\|\partial \phi\left(t_{0}\right)\right\|_{L^{2}\left(\Sigma_{t_{0}}\right)}
$$

This estimate allows us to write (131) in the form:

$$
E\left(\partial_{t} \phi\right)(t) \lesssim \int_{\Sigma_{t}} \bar{Q}\left(K, \partial_{t}\right)+E\left(\partial_{t} \phi\right)\left(t_{0}\right)
$$

Checking easily that $\square_{g}(t)=g^{i j} \kappa_{i j}$, the integral term $-\frac{1}{2} \int_{\left[t_{0}, t\right] \times \mathbb{R}^{2}}\left(\partial_{t} \phi\right)^{2} \square_{g}(\tau)$ can be estimated as:

$$
\begin{aligned}
\left|-\frac{1}{2} \int_{\left[t_{0}, t\right] \times \mathbb{R}^{2}}\left(\partial_{t} \phi\right)^{2} \square_{g}(\tau)\right| & \lesssim \int_{t_{0}}^{t}\left\|\partial_{t} \phi(t)\right\|_{L^{2}\left(\Sigma_{\tau}\right)}^{2}\left\|g^{i j} \kappa_{i j}\right\|_{L^{\infty}\left(\Sigma_{\tau}\right)} d \tau \\
& \lesssim \lambda^{-\epsilon} \sup _{\tau \in\left[t_{0}, t_{*}\right]} E\left(\partial_{t} \phi\right)(\tau)
\end{aligned}
$$

Putting together (133) and (134) with the obvious inequality

$$
\int_{\Sigma_{t_{0}}} \bar{Q}\left(K, \partial_{t}\right) \lesssim E\left(\partial_{t} \phi\right)\left(t_{0}\right)
$$

we obtain (128).

Proposition 7.5 The deformation tensor ${ }^{K} \pi$ of the Morawetz vectorfield

$$
K=\frac{1}{2}\left(u^{2} \underline{L}+\underline{u}^{2} L\right),
$$

satisfies

$$
\begin{aligned}
& { }^{K} \pi_{L L}=-2 u^{2} \kappa_{N N} \\
& { }^{K} \pi_{\underline{L L}}=2 \underline{u}\left(4\left(b^{-1}-1\right)-\underline{u} \kappa_{N N}\right) \\
& { }^{K} \pi_{A A}=u^{2} \underline{\chi}+\underline{u}^{2} \chi \\
& { }^{K} \pi_{L \underline{L}}=-4\left(\underline{u}+b^{-1} u\right)+\left(u^{2}+\underline{u}^{2}\right) \kappa_{N N} \\
& { }^{K} \pi_{L A}=-2 u^{2} \kappa_{A N} \\
& { }^{K} \pi_{\underline{L A}}=u^{2} \underline{\xi}+\underline{u}^{2}\left(\eta+\kappa_{A N}\right)
\end{aligned}
$$

Proof Using the formula for $K$, we can write:

$$
\begin{aligned}
{ }^{K} \pi_{\alpha \beta} & =\frac{1}{2} u^{2}\left(<\mathcal{D} \alpha \underline{L}, \partial_{\beta}>+<\mathcal{D}_{\beta} \underline{L}, \partial_{\alpha}>\right)+\frac{1}{2} \underline{u}^{2}\left(<\mathcal{D}_{\alpha} L, \partial_{\beta}>+<\mathcal{D}_{\beta} L, \partial_{\alpha}>\right)+ \\
& +\frac{1}{2} \partial_{\alpha} u^{2}<\underline{L}, \partial_{\beta}>+\frac{1}{2} \partial_{\beta} u^{2}<\underline{L}, \partial_{\alpha}>+\frac{1}{2} \partial_{\alpha} \underline{u}^{2}<L, \partial_{\beta}>+\frac{1}{2} \partial_{\beta} \underline{u}^{2}<L, \partial_{\alpha}>
\end{aligned}
$$


This implies

$$
\begin{aligned}
& { }^{K} \pi_{L L}=u^{2}<\mathcal{D}_{L} \underline{L}, L>+\underline{u}^{2}<\mathcal{D}_{L} L, L>+L\left(u^{2}\right)<\underline{L}, L>+L\left(\underline{u}^{2}\right)<L, L>= \\
& =-2 u^{2} \kappa_{N N} \\
& { }^{K} \pi_{\underline{L L}}=u^{2}<\mathcal{D}_{\underline{L}} \underline{L}, \underline{L}>+\underline{u}^{2}<\mathcal{D}_{\underline{L}} L, \underline{L}>+\underline{L}\left(u^{2}\right)<\underline{L}, L>+\underline{L}\left(\underline{u}^{2}\right)<L, L>= \\
& =\underline{u}^{2}\left(-2 \kappa_{N N}\right)+(-4 \underline{u})\left(2-2 b^{-1}\right)=2 \underline{u}\left(4\left(b^{-1}-1\right)-\underline{u} \kappa_{N N}\right) \\
& { }^{K} \pi_{A A}=u^{2}<\mathcal{D}_{A} \underline{L}, A>+\underline{u}^{2}<\mathcal{D}_{A} L, A>+A\left(u^{2}\right)<\underline{L}, A>+A\left(\underline{u}^{2}\right)<L, A>= \\
& =u^{2} \underline{\chi}+\underline{u}^{2} \chi \\
& { }^{K} \pi_{L A}=\frac{1}{2} u^{2}\left(<\mathcal{D}_{L} \underline{L}, A>+<\mathcal{D}_{A} \underline{L}, L>\right)+\frac{1}{2} \underline{u}^{2}\left(<\mathcal{D}_{L} L, A>+<\mathcal{D}_{A} L, L>\right)+ \\
& +\frac{1}{2} L\left(u^{2}\right)<\underline{L}, A>+\frac{1}{2} A\left(u^{2}\right)<\underline{L}, L>+\frac{1}{2} L\left(\underline{u}^{2}\right)<L, A>+\frac{1}{2} A\left(\underline{u}^{2}\right)<L, L>= \\
& =\frac{1}{2} u^{2}\left(2 \underline{\eta}-2 \kappa_{A N}\right)=-2 u^{2} \kappa_{A N} \\
& { }^{K} \pi_{\underline{L A}}=\frac{1}{2} u^{2}\left(<\mathcal{D}_{\underline{L}} \underline{L}, A>+<\mathcal{D}_{A} \underline{L}, \underline{L}>\right)+\frac{1}{2} \underline{u}^{2}\left(<\mathcal{D}_{\underline{L}} L, A>+<\mathcal{D}_{A} L, \underline{L}>\right)+ \\
& +\frac{1}{2} \underline{L}\left(u^{2}\right)<\underline{L}, A>+\frac{1}{2} A\left(u^{2}\right)<\underline{L}, \underline{L}>+\frac{1}{2} \underline{L}\left(\underline{u}^{2}\right)<L, A>+\frac{1}{2} A\left(\underline{u}^{2}\right)<L, \underline{L}>= \\
& =u^{2} \underline{\xi}+\underline{u}^{2}\left(\eta+\kappa_{A N}\right) \\
& K^{K} \pi_{L \underline{L}}=\frac{1}{2} u^{2}\left(<\mathcal{D}_{L} \underline{L}, \underline{L}>+<\mathcal{D}_{\underline{L}} \underline{L}, L>\right)+\frac{1}{2} \underline{u}^{2}\left(<\mathcal{D}_{L} L, \underline{L}>+<\underline{\mathcal{D}}_{\underline{L}} L, L>\right)+ \\
& +\frac{1}{2} L\left(u^{2}\right)<\underline{L}, \underline{L}>+\frac{1}{2} \underline{L}\left(u^{2}\right)<\underline{L}, L>+\frac{1}{2} L\left(\underline{u}^{2}\right)<L, \underline{L}>+\frac{1}{2} \underline{L}\left(\underline{u}^{2}\right)<L, L>= \\
& =u^{2} \kappa_{N N}+\underline{u^{2}} \kappa_{N N}-4 u b^{-1}-4 \underline{u}
\end{aligned}
$$

This concludes the proof of this proposition. Next, we will analyze the integral term in (128) which involves the deformation tensor ${ }^{K} \pi$. In this direction, we can prove the next

Lemma 7.6 Under the assumptions of Theorem 5.1:

$$
\left|\int_{\left[t_{0}, t\right] \times \mathbb{R}^{2}}\left(\frac{1}{2} Q^{\alpha \beta K} \tilde{\pi}_{\alpha \beta}-\frac{1}{4} \underline{u}^{2}\left(\chi-\frac{1}{s}\right) \partial_{t} \phi \cdot \square_{g}\left(\partial_{t} \phi\right) \cdot \omega\right)\right| \lesssim \lambda^{-\epsilon} \sup _{\tau \in\left[t_{0}, t_{*}\right]} E(\partial \phi)(\tau)
$$

Proof Detailing the term $Q^{\alpha \beta K} \tilde{\pi}_{\alpha \beta}$, we obtain:

$$
\begin{aligned}
Q^{\alpha \beta K} \tilde{\pi}_{\alpha \beta} & =\frac{1}{4}\left({ }^{K} \tilde{\pi}_{L L}\left|\underline{L} \partial_{t} \phi\right|^{2}+{ }^{K} \tilde{\pi}_{\underline{L L}}\left|L \partial_{t} \phi\right|^{2}\right)+\frac{1}{2}\left({ }^{K} \tilde{\pi}_{A A}\left(L \partial_{t} \phi \cdot \underline{L} \partial_{t} \phi+\left|A \partial_{t} \phi\right|^{2}\right)+\right. \\
& \left.+{ }^{K} \tilde{\pi}_{L \underline{L}}\left|A \partial_{t} \phi\right|^{2}\right)-{ }^{K} \tilde{\pi}_{L A} A \partial_{t} \phi \cdot \underline{L} \partial_{t} \phi-{ }^{K} \tilde{\pi}_{\underline{L A}} A \partial_{t} \phi \cdot L \partial_{t} \phi
\end{aligned}
$$

Using the expressions for the deformation tensor of $K$ provided by Proposition 7.5, we have the following set of estimates:

$$
\begin{aligned}
&\left.\left|{ }^{K} \tilde{\pi}_{L L}\right| \underline{L} \partial_{t} \phi\right|^{2} \mid \lesssim \lambda^{-\bar{a}}\left|u \underline{L} \partial_{t} \phi\right|^{2} \\
&\left.\left.\left|{ }^{K} \tilde{\pi}_{\underline{L L}}\right| L \partial_{t} \phi\right|^{2}\left|\lesssim \lambda^{-\bar{a}}\right| \underline{u} L \partial_{t} \phi\right|^{2} \\
&\left.\left.\left|{ }^{K} \tilde{\pi}_{A A}\right| A \partial_{t} \phi\right|^{2}\left|\lesssim \lambda^{-\bar{a}}\right| \underline{u} A \partial_{t} \phi\right|^{2}
\end{aligned}
$$




$$
\begin{aligned}
& \left.\left.\left|{ }^{K} \tilde{\pi}_{L \underline{L}}\right| A \partial_{t} \phi\right|^{2}\left|\lesssim \lambda^{-\bar{a}}\right| \underline{u} A \partial_{t} \phi\right|^{2} \\
& \left|{ }^{K} \tilde{\pi}_{L A} A \partial_{t} \phi \cdot \underline{L} \partial_{t} \phi\right| \lesssim \lambda^{-\bar{a}}\left|u \underline{L} \partial_{t} \phi\right| \cdot\left|\underline{u} A \partial_{t} \phi\right| \\
& \left|{ }^{K} \tilde{\pi}_{\underline{L} A} A \partial_{t} \phi \cdot L \partial_{t} \phi\right| \lesssim\left(\lambda^{-\bar{a}}+\tau^{\frac{1}{2}} \lambda^{-\bar{a}-\frac{1-a}{2}+\epsilon}\right)\left|\underline{u} L \partial_{t} \phi\right| \cdot\left|\underline{u} A \partial_{t} \phi\right|
\end{aligned}
$$

These estimates imply directly:

$$
\left|\int_{\left[t_{0}, t\right] \times \mathbb{R}^{2}}\left(\frac{1}{2} Q^{\alpha \beta K} \tilde{\pi}_{\alpha \beta}-\frac{1}{4} K_{\tilde{\pi}_{A A}} L \partial_{t} \phi \cdot \underline{L} \partial_{t} \phi\right)\right| \lesssim \lambda^{-\epsilon} \sup _{\tau \in\left[t_{0}, t_{*}\right]} E(\partial \phi)(\tau) .
$$

Due to the fact that

$$
K_{\tilde{\pi}_{A A}}=u^{2}\left(\underline{\chi}+\frac{1}{s}\right)+\underline{u}^{2}\left(\chi-\frac{1}{s}\right)
$$

and because

$$
\begin{aligned}
& \left|u^{2}\left(\underline{\chi}+\frac{1}{s}\right) L \partial_{t} \phi \cdot \underline{L} \partial_{t} \phi\right| \lesssim \lambda^{-\bar{a}}\left|u \underline{L} \partial_{t} \phi\right| \cdot\left|\underline{u} L \partial_{t} \phi\right| \\
& \left|{ }^{K} \tilde{\pi}_{A A} L \partial_{t} \phi \cdot \underline{L} \partial_{t} \phi \cdot(1-\omega)\right| \lesssim \lambda^{-\bar{a}}\left|\tau \partial \partial_{t} \phi \cdot(1-\omega)\right|^{2}
\end{aligned}
$$

we can rewrite (136) in the form:

$$
\left|\int_{\left[t_{0}, t\right] \times \mathbb{R}^{2}}\left(\frac{1}{2} Q^{\alpha \beta K} \tilde{\pi}_{\alpha \beta}-\frac{1}{4} \underline{u}^{2}\left(\chi-\frac{1}{s}\right) L \partial_{t} \phi \cdot \underline{L} \partial_{t} \phi \cdot \omega\right)\right| \lesssim \lambda^{-\epsilon} \sup _{\tau \in\left[t_{0}, t_{*}\right]} E(\partial \phi)(\tau) .
$$

We are in a position to apply one of our integration results, (83), for the second term in the above integral. It will follow that:

$$
\begin{aligned}
\int_{\left[t_{0}, t\right] \times \mathbb{R}^{2}} \frac{1}{4} \underline{u}^{2}\left(\chi-\frac{1}{s}\right) L \partial_{t} \phi \cdot \underline{L} \partial_{t} \phi \cdot \omega= & \int_{\left[t_{0}, t\right] \times \mathbb{R}^{2}} \frac{1}{4} \underline{u}^{2}\left(\chi-\frac{1}{s}\right) \partial_{t} \phi \cdot \square_{g}\left(\partial_{t} \phi\right) \cdot \omega+ \\
& +O\left(\lambda^{-\epsilon}\right) \sup _{\tau \in\left[t_{0}, t_{*}\right]} E(\partial \phi)(\tau)
\end{aligned}
$$

provided the conditions set in Proposition 3.10 are fulfilled. Let us verify them using the asymptotic estimates:

$$
\begin{aligned}
\left|\frac{1}{4} \underline{u}^{2}\left(\chi-\frac{1}{s}\right)\right| & \lesssim \tau^{2} \cdot \lambda^{-\bar{a}} \lesssim \tau \cdot \lambda^{-\epsilon} \\
\int_{t_{0}}^{t} \frac{1}{\tau^{\frac{3}{2}}} \cdot \sup _{0 \leq u \leq \frac{t}{2}}\left\|\not \nabla\left(\frac{1}{4} \underline{u}^{2}\left(\chi-\frac{1}{s}\right)\right)\right\|_{L^{2}\left(S_{\tau, u}\right)} d \tau & \lesssim \int_{t_{0}}^{t} \tau^{\frac{1}{2}}\left(\lambda^{-\bar{a}-\frac{1-a}{2}+\epsilon}+\tau^{-\frac{1}{2}} \cdot \lambda^{-\bar{a}}\right) d \tau \\
& \lesssim \lambda^{-\epsilon} \\
\int_{t_{0}}^{t} \frac{1}{\tau^{\frac{3}{2}}} \sup _{0 \leq u \leq \frac{t}{2}}\left\|\underline{L}\left(\frac{1}{4} \underline{u}^{2}\left(\chi-\frac{1}{s}\right)\right)\right\|_{L^{2}\left(S_{\tau, u}\right)} d \tau & \lesssim \int_{t_{0}}^{t} \tau^{\frac{1}{2}}\left(\lambda^{-\bar{a}-\frac{1-a}{2}+\epsilon}+\tau^{-\frac{1}{2}} \cdot \lambda^{-\bar{a}}+\right. \\
& \left.+\tau^{\frac{1}{2}} \cdot \lambda^{-2 \bar{a}}\right) d \tau \lesssim \lambda^{-\epsilon}
\end{aligned}
$$

This concludes, of course, the proof of our lemma. Having in mind (128) and (135), in order to obtain (123), we are left to prove:

$$
\left|\int_{\left[t_{0}, t\right] \times \mathbb{R}^{2}}\left(K \partial_{t} \phi+\tau \partial_{t} \phi+\frac{1}{4} \underline{u}^{2}\left(\chi-\frac{1}{s}\right) \partial_{t} \phi \cdot \omega\right) \square_{g}\left(\partial_{t} \phi\right)\right| \lesssim \lambda^{-\epsilon} \sup _{\tau \in\left[t_{0}, t_{*}\right]} E(\partial \phi)(\tau) .
$$


Let us concentrate for a moment on $\square_{g}\left(\partial_{t} \phi\right)$. We have the formula:

$$
\square_{g}\left(\partial_{t} \phi\right)=-\partial_{t} g^{i j} \cdot \partial_{i} \partial_{j} \phi-\partial_{t}(t r \kappa) \cdot \partial_{t} \phi-\partial_{t}\left(\Gamma_{i i}^{j}\right) \cdot \partial_{j} \phi
$$

Due to this formula we can split $\square_{g}\left(\partial_{t} \phi\right)$ into two parts: $A$ which contains only second order derivatives for $\phi$ and $B$ which gathers the first order derivatives of $\phi$. Accordingly, we will prove (139) by considering first the part related to $B$ and then the part related to $A$. We do it like this because some of the information obtained by proving the " $B$-part" will be used in proving the "A-part". We will further divide our argument into the one concerning the interior part of the integral and the one concerning its exterior part.

\subsection{Estimate of the B-part}

Due to the formula (140):

$$
B=-\partial_{t}(t r \kappa) \cdot \partial_{t} \phi-\partial_{t}\left(\Gamma_{i i}^{j}\right) \cdot \partial_{j} \phi=\left(\left|\partial^{2} g\right|+|\partial g|^{2}\right) \cdot \partial \phi
$$

First we prove the interior estimate:

Proposition 7.7 Under the assumptions of Theorem 5.1:

$$
\left.\mid \int_{\left[t_{0}, t\right] \times \mathbb{R}^{2}}\left(K \partial_{t} \phi+\tau \partial_{t} \phi\right) \cdot(1-\omega)\right) B \mid \lesssim \lambda^{-\epsilon} \sup _{\tau \in\left[t_{0}, t_{*}\right]} E(\partial \phi)(\tau) .
$$

Proof Estimating directly we obtain

$$
\begin{aligned}
& \left|\int_{\left[t_{0}, t\right] \times \mathbb{R}^{2}}\left(K \partial_{t} \phi+\tau \partial_{t} \phi\right)(1-\omega) B\right|=\left|\int_{\left[t_{0}, t\right] \times \mathbb{R}^{2}}\left(u^{2} \underline{L}\left(\partial_{t} \phi\right)+\underline{u}^{2} L\left(\partial_{t} \phi\right)+\tau \partial_{t} \phi\right) \cdot(1-\omega) \cdot B\right| \\
& \lesssim \int_{t_{0}}^{t} \tau \cdot E^{\frac{1}{2}}(\partial \phi)(\tau) \cdot\left\|\left(\left|\partial^{2} g\right|+|\partial g|^{2}\right) \cdot \partial \phi \cdot(1-\omega)\right\|_{L^{2}\left(\Sigma_{\tau}\right)} d \tau \lesssim \\
& \lesssim \int_{t_{0}}^{t} \tau E^{\frac{1}{2}}(\partial \phi)(\tau)\left\|\partial^{2} g \partial \phi(1-\omega)\right\|_{L^{2}\left(\Sigma_{\tau}\right)} d \tau+\int_{t_{0}}^{t} \tau E^{\frac{1}{2}}(\partial \phi)(\tau)\left\||\partial g|^{2} \cdot \partial \phi(1-\omega)\right\|_{L^{2}\left(\Sigma_{\tau}\right)} d \tau
\end{aligned}
$$

The second term is easily estimated as follows:

$$
\begin{aligned}
& \int_{t_{0}}^{t} \tau \cdot E^{\frac{1}{2}}(\partial \phi)(\tau) \cdot\left\||\partial g|^{2} \cdot \partial \phi(1-\omega)\right\|_{L^{2}\left(\Sigma_{\tau}\right)} d \tau \lesssim \\
& \lesssim \int_{t_{0}}^{t} \tau \cdot E^{\frac{1}{2}}(\partial \phi)(\tau) \cdot\left\||\partial g|^{2}\right\|_{L^{\infty}\left(\Sigma_{t}\right)} \cdot\|\partial \phi(1-\omega)\|_{L^{2}\left(\Sigma_{\tau}\right)} d \tau \lesssim \int_{t_{0}}^{t} \tau \cdot \lambda^{-2 \bar{a}} \cdot E(\partial \phi)(\tau) d \tau \lesssim \\
& \lesssim \lambda^{-\epsilon} \sup _{\tau \in\left[t_{0}, t_{*}\right]} E(\partial \phi)(\tau)
\end{aligned}
$$


while for the first one we have to be more subtle in our approach, because we do not control $\|\partial \phi(1-\omega)\|_{L^{\infty}\left(\Sigma_{t}\right)}$. We proceed like this:

$$
\begin{aligned}
\int_{t_{0}}^{t} \tau \cdot E^{\frac{1}{2}}(\partial \phi)(\tau) \cdot\left\|\partial^{2} g \cdot \partial \phi \cdot(1-\omega)\right\|_{L^{2}\left(\Sigma_{\tau}\right)} d \tau & \lesssim \\
& \lesssim \int_{t_{0}}^{t} \tau \cdot E^{\frac{1}{2}}(\partial \phi)(\tau) \cdot\left\|\partial^{2} g\right\|_{L^{p}\left(\Sigma_{\tau}\right)} \cdot\|\partial \phi(1-\omega)\|_{L^{q}\left(\Sigma_{\tau}\right)} d \tau
\end{aligned}
$$

where

$$
\frac{1}{p}+\frac{1}{q}=\frac{1}{2}
$$

Due to Bernstein inequality we have the estimate:

$$
\left\|\partial^{2} g\right\|_{L^{p}\left(\Sigma_{\tau}\right)} \lesssim \lambda^{\frac{1}{2}-\frac{1}{p}}\left\|\partial^{2} g\right\|_{L^{2}\left(\Sigma_{\tau}\right)}
$$

Using Sobolev inequality we obtain:

$$
\|\partial \phi \cdot(1-\omega)\|_{L^{q}\left(\Sigma_{\tau}\right)} \lesssim\left\|\partial^{2-\frac{2}{q}} \phi \cdot(1-\omega)\right\|_{L^{2}\left(\Sigma_{\tau}\right)} .
$$

Estimating the last term by interpolation, we infer that:

$$
\begin{aligned}
\left\|\partial^{2-\frac{2}{q}} \phi \cdot(1-\omega)\right\|_{L^{2}\left(\Sigma_{\tau}\right)} & \lesssim\left\|\partial^{2} \phi \cdot(1-\omega)\right\|_{L^{2}\left(\Sigma_{\tau}\right)}^{1-\frac{2}{q}} \cdot\|\partial \phi \cdot(1-\omega)\|_{L^{2}\left(\Sigma_{\tau}\right)}^{\frac{2}{q}} \lesssim \\
& \lesssim \tau^{-1+\frac{2}{q}} \cdot E^{\frac{1}{2}}(\partial \phi)(\tau) .
\end{aligned}
$$

Putting altogether the last four estimates, we obtain:

$$
\begin{aligned}
\int_{t_{0}}^{t} \tau \cdot E^{\frac{1}{2}}(\partial \phi)(\tau) \cdot\left\|\partial^{2} g \cdot \partial \phi \cdot(1-\omega)\right\|_{L^{2}\left(\Sigma_{\tau}\right)} d \tau & \lesssim \int_{t_{0}}^{t} \tau^{\frac{2}{q}} \cdot \lambda^{\frac{1}{q}-\bar{a}} \cdot E(\partial \phi)(\tau) d \tau \lesssim \\
& \lesssim \lambda^{-\epsilon} \sup _{\tau \in\left[t_{0}, t_{*}\right]} E(\partial \phi)(\tau)
\end{aligned}
$$

for $q$ large enough, which ends the proof of this result. Therefore, we are left to prove that:

Proposition 7.8 Under the assumptions of Theorem 5.1:

$$
\left|\int_{\left[t_{0}, t\right] \times \mathbb{R}^{2}}\left(K \partial_{t} \phi+\left(\tau+\frac{1}{4} \underline{u}^{2}\left(\chi-\frac{1}{s}\right)\right) \partial_{t} \phi\right) \cdot \omega \cdot B\right| \lesssim \lambda^{-\epsilon} \sup _{\tau \in\left[t_{0}, t_{*}\right]} E(\partial \phi)(\tau) .
$$

Proof Using the integral estimate (82) corresponding to the exterior region, we obtain:

$$
\begin{aligned}
\mid \int_{\left[t_{0}, t\right] \times \mathbb{R}^{2}} & \left(K \partial_{t} \phi+\left(\tau+\frac{1}{4} \underline{u}^{2}\left(\chi-\frac{1}{s}\right)\right) \partial_{t} \phi\right) \cdot \omega \cdot B \mid \lesssim \\
& \lesssim \int_{t_{0}}^{t} \tau \cdot E^{\frac{1}{2}}(\partial \phi)(\tau) \cdot\left\|\left(\left|\partial^{2} g\right|+|\partial g|^{2}\right) \cdot \partial \phi \cdot \omega\right\|_{L^{2}\left(\Sigma_{\tau}\right)} d \tau \lesssim \\
& \lesssim \int_{t_{0}}^{t} \tau^{\frac{1}{2}} \cdot \sup _{0 \leq u \leq \frac{\tau}{2}}\left\|\left|\partial^{2} g\right|+|\partial g|^{2}\right\|_{L^{2}\left(S_{\tau, u}\right)} \cdot E(\partial \phi)(\tau) d \tau \lesssim
\end{aligned}
$$




$$
\begin{aligned}
& \lesssim \int_{t_{0}}^{t} \tau^{\frac{1}{2}} \cdot\left(\lambda^{-\bar{a}-\frac{1-a}{2}+\epsilon}+\tau^{\frac{1}{2}} \lambda^{-2 \bar{a}}\right) \cdot E(\partial \phi)(\tau) d \tau \lesssim \\
& \lesssim \lambda^{-\epsilon} \sup _{\tau \in\left[t_{0}, t_{*}\right]} E(\partial \phi)(\tau) .
\end{aligned}
$$

This concludes the discussion concerning $B$.

\subsection{Estimate of the A-part}

According to formula (140)

$$
A=-\partial_{t} g^{i j} \cdot \partial_{i} \partial_{j} \phi=\partial g \cdot \partial^{2} \phi
$$

We will prove that:

Proposition 7.9 Under the assumptions of Theorem 5.1 :

$$
\left.\mid \int_{\left[t_{0}, t\right] \times \mathbb{R}^{2}}\left(K \partial_{t} \phi+\tau \partial_{t} \phi\right) \cdot(1-\omega)\right) A \mid \lesssim \lambda^{-\epsilon} \sup _{\tau \in\left[t_{0}, t_{*}\right]} E(\partial \phi)(\tau) .
$$

Proof Estimating directly, as in Proposition 7.7, we obtain

$$
\begin{aligned}
\mid \int_{\left[t_{0}, t\right] \times \mathbb{R}^{2}} & \left.\left(K \partial_{t} \phi+\tau \partial_{t} \phi\right) \cdot(1-\omega)\right) A \mid \lesssim \\
& \lesssim \int_{t_{0}}^{t} \tau \cdot E^{\frac{1}{2}}(\partial \phi)(\tau) \cdot\left\||\partial g| \cdot\left|\partial^{2} \phi\right| \cdot(1-\omega)\right\|_{L^{2}\left(\Sigma_{\tau}\right)} d \tau \lesssim \\
& \lesssim \int_{t_{0}}^{t}\|\partial g\|_{L^{\infty}\left(\Sigma_{\tau}\right)} \cdot E(\partial \phi)(\tau) d \tau \lesssim \lambda^{-\epsilon} \sup _{\tau \in\left[t_{0}, t_{*}\right]} E(\partial \phi)(\tau)
\end{aligned}
$$

We investigate now the exterior part of the integral. Due to the fact that in the exterior region, in the energy expression, all the terms but the $\underline{L}$-derivative come with a weight comparable to $\tau$, we can infer that:

$$
\begin{aligned}
\mid \int_{\left[t_{0}, t\right] \times \mathbb{R}^{2}}\left(K \partial_{t} \phi\right. & \left.+\left(\tau+\frac{1}{4} \underline{u}^{2}\left(\chi-\frac{1}{s}\right)\right) \partial_{t} \phi\right) \cdot \omega \cdot A^{\prime} \mid \lesssim \\
& \lesssim \int_{t_{0}}^{t} \tau \cdot E^{\frac{1}{2}}(\partial \phi)(\tau) \cdot\left\|A^{\prime} \cdot \omega\right\|_{L^{2}\left(\Sigma_{\tau}\right)} d \tau \lesssim \\
& \lesssim \int_{t_{0}}^{t}\|\partial g\|_{L^{\infty}\left(\Sigma_{\tau}\right)} \cdot E(\partial \phi)(\tau) d \tau \lesssim \lambda^{-\epsilon} \sup _{\tau \in\left[t_{0}, t_{*}\right]} E(\partial \phi)(\tau),
\end{aligned}
$$

where $A^{\prime}$ differs from $A$ just through the term which involves two $\underline{L}$-derivatives on $\phi$. We can further simplify our investigation by replacing $\mathcal{D}_{\underline{L}} \mathcal{D}_{\underline{L}} \phi=\mathcal{D}_{\underline{L}} \mathcal{D}_{2 T-L} \phi$ with $\underline{L}\left(\partial_{t} \phi\right)$, because the difference between these two terms is of $A^{\prime}$-type. Therefore, the expression to estimate is:

$$
\int_{\left[t_{0}, t\right] \times \mathbb{R}^{2}}\left(K \partial_{t} \phi+\left(\tau+\frac{1}{4} \underline{u}^{2}\left(\chi-\frac{1}{s}\right)\right) \partial_{t} \phi\right) \cdot \omega \cdot A_{\underline{L}} \cdot \underline{L}\left(\partial_{t} \phi\right),
$$


where $A_{\underline{L}}={ }^{T} \pi_{L L}=-2 \kappa_{N N}$. This is the coefficient of $\mathcal{D}_{\underline{L}} \mathcal{D}_{\underline{L}} \phi$ in the expression of $A$, as it can easily by commuting the wave operator $\square_{g}$ with the vectorfield $T=\partial_{t}$. Using the trivial estimate $\left|A_{\underline{L}}\right| \lesssim|\partial g|$, we immediately obtain:

$$
\begin{aligned}
\mid \int_{\left[t_{0}, t\right] \times \mathbb{R}^{2}} u^{2} \cdot \underline{L}\left(\partial_{t} \phi\right) & \cdot A_{\underline{L}} \cdot \underline{L}\left(\partial_{t} \phi\right) \mid \lesssim \\
& \lesssim \int_{t_{0}}^{t}\|\partial g\|_{L^{\infty}\left(\Sigma_{\tau}\right)} \cdot E(\partial \phi)(\tau) d \tau \lesssim \lambda^{-\epsilon} \sup _{\tau \in\left[t_{0}, t_{*}\right]} E(\partial \phi)(\tau) .
\end{aligned}
$$

We will investigate now the term

$$
\int_{\left[t_{0}, t\right] \times \mathbb{R}^{2}}\left(\tau+\frac{1}{4} \underline{u}^{2}\left(\chi-\frac{1}{s}\right)\right) \partial_{t} \phi \cdot \omega \cdot A_{\underline{L}} \cdot \underline{L}\left(\partial_{t} \phi\right)
$$

Again, we are in a position to apply one of our integration estimates, (84), provided $\left(\tau+\frac{1}{4} \underline{u}^{2}\left(\chi-\frac{1}{s}\right)\right) \cdot A_{\underline{L}}$ verifies the conditions imposed in Proposition 3.10. We check them one by one:

$$
\begin{gathered}
\left|\left(\tau+\frac{1}{4} \underline{u}^{2}\left(\chi-\frac{1}{s}\right)\right) \cdot A_{\underline{L}}\right| \lesssim\left(\tau+\tau^{2} \lambda^{-\bar{a}}\right) \cdot|\partial g| \lesssim \lambda^{-\epsilon} \\
\int_{t_{0}}^{t} \frac{1}{\tau^{\frac{1}{2}}} \cdot \sup _{0 \leq u \leq \frac{\tau}{2}}\left\|\underline{L}\left(\left(\tau+\frac{1}{4} \underline{u}^{2}\left(\chi-\frac{1}{s}\right)\right) \cdot A_{\underline{L}}\right)\right\|_{L^{2}\left(S_{\tau, u}\right)} d \tau \lesssim \\
\lesssim \int_{t_{0}}^{t} \frac{1}{\tau^{\frac{1}{2}}}\left(\lambda^{-\bar{a}} \sup _{0 \leq u \leq \frac{\tau}{2}}\left\|\underline{L}\left(\tau+\frac{1}{4} \underline{u}^{2}\left(\chi-\frac{1}{s}\right)\right)\right\|_{L^{2}\left(S_{\tau, u}\right)}+\left(\tau+\tau^{2} \lambda^{-\bar{a}}\right) \sup _{0 \leq u \leq \frac{\tau}{2}}\left\|\underline{L}\left(A_{\underline{L}}\right)\right\|_{L^{2}\left(S_{\tau, u}\right)}\right) d \tau \\
\lesssim \int_{t_{0}}^{t} \frac{1}{\tau^{\frac{1}{2}}} \cdot\left(\tau^{\frac{1}{2}} \lambda^{-\bar{a}}+\tau \lambda^{-\bar{a}-\frac{1-a}{2}+\epsilon}+\tau^{\frac{3}{2}} \lambda^{-2 \bar{a}}+\tau^{2} \lambda^{-2 \bar{a}-\frac{1-a}{2}+\epsilon}\right) d \tau \\
\lesssim \lambda^{-\epsilon} \sup _{\tau \in\left[t_{0}, t_{*}\right]} E(\partial \phi)(\tau)
\end{gathered}
$$

This allows to conclude:

$$
\left|\int_{\left[t_{0}, t\right] \times \mathbb{R}^{2}}\left(\tau+\frac{1}{4} \underline{u}^{2}\left(\chi-\frac{1}{s}\right)\right) \partial_{t} \phi \cdot \omega \cdot A_{\underline{L}} \cdot \underline{L}\left(\partial_{t} \phi\right)\right| \lesssim \lambda^{-\epsilon} \sup _{\tau \in\left[t_{0}, t_{*}\right]} E(\partial \phi)(\tau)
$$

Remark Already at this stage with the estimates obtained so far, we have:

$$
\left|\int_{\left[t_{0}, t\right] \times \mathbb{R}^{2}}\left(\tau+\frac{1}{4} \underline{u}^{2}\left(\chi-\frac{1}{s}\right)\right) \cdot \omega \cdot \partial_{t} \phi \cdot \square_{g}\left(\partial_{t} \phi\right)\right| \lesssim \lambda^{-\epsilon} \sup _{\tau \in\left[t_{0}, t_{*}\right]} E(\partial \phi)(\tau)
$$

The last term to consider is

$$
\int_{\left[t_{0}, t\right] \times \mathbb{R}^{2}} \underline{u}^{2} \cdot L\left(\partial_{t} \phi\right) \cdot A_{\underline{L}} \cdot \underline{L}\left(\partial_{t} \phi\right) \cdot \omega
$$

We will use once again (83). The conditions to be satisfied are:

$$
\left|\underline{u}^{2} A_{\underline{L}}\right| \lesssim \tau^{2} \cdot \lambda^{-\bar{a}} \lesssim \tau \cdot \lambda^{-\epsilon}
$$




$$
\begin{gathered}
\int_{t_{0}}^{t} \frac{1}{\tau^{\frac{3}{2}}} \cdot \sup _{0 \leq u \leq \frac{t}{2}}\left\|\not \nabla\left(\underline{u}^{2} A_{\underline{L}}\right)\right\|_{L^{2}\left(S_{\tau, u}\right)} d \tau \lesssim \int_{t_{0}}^{t}\left(\tau^{\frac{1}{2}} \lambda^{-\bar{a}-\frac{1-a}{2}+\epsilon}+\lambda^{-\bar{a}}\right) d \tau \lesssim \lambda^{-\epsilon} \\
\int_{t_{0}}^{t} \frac{1}{\tau^{\frac{3}{2}}} \sup _{0 \leq u \leq \frac{t}{2}}\left\|\underline{L}\left(\underline{u}^{2} A_{\underline{L}}\right)\right\|_{L^{2}\left(S_{\tau, u}\right)} d \tau \lesssim \int_{t_{0}}^{t}\left(\tau^{\frac{1}{2}} \lambda^{-\bar{a}-\frac{1-a}{2}+\epsilon}+\lambda^{-\bar{a}}\right) d \tau \lesssim \lambda^{-\epsilon}
\end{gathered}
$$

Therefore, we can write:

$$
\begin{aligned}
& \int_{\left[t_{0}, t\right] \times \mathbb{R}^{2}} \underline{u}^{2} \cdot L\left(\partial_{t} \phi\right) \cdot A_{\underline{L}} \cdot \underline{L}\left(\partial_{t} \phi\right) \cdot \omega= \\
& =\int_{\left[t_{0}, t\right] \times \mathbb{R}^{2}} \underline{u}^{2} \cdot A_{\underline{L}} \cdot \omega \cdot \partial_{t} \phi \cdot \square_{g}\left(\partial_{t} \phi\right)+O\left(\lambda^{-\epsilon}\right) \sup _{\tau \in\left[t_{0}, t_{*}\right]} E(\partial \phi)(\tau)
\end{aligned}
$$

Taking into account the previous Remark and the fact that $\underline{u}^{2} A_{\underline{L}}$ is "dominated" by $\tau+\frac{1}{4} \underline{u}^{2}\left(\chi-\frac{1}{s}\right)$, we claim that we will also obtain

$$
\left|\int_{\left[t_{0}, t\right] \times \mathbb{R}^{2}} \underline{u}^{2} \cdot A_{\underline{L}} \cdot \omega \cdot \partial_{t} \phi \cdot \square_{g}\left(\partial_{t} \phi\right)\right| \lesssim \lambda^{-\epsilon} \sup _{\tau \in\left[t_{0}, t_{*}\right]} E(\partial \phi)(\tau) .
$$

This concludes the argument for this section.

\section{Energy estimates involving the scaling vectorfield $S$ and the angular momentum vectorfield $\Omega$}

The main result of this section is:

Theorem 8.1 Under the assumptions of Theorem 5.1:

$$
\begin{aligned}
\|\partial S \phi\|_{L^{2}\left(\Sigma_{t}\right)} & \lesssim\|\partial S \phi\|_{L^{2}\left(\Sigma_{t_{0}}\right)}+\lambda^{-\epsilon} \sup _{\tau \in\left[t_{0}, t_{*}\right]} E^{\frac{1}{2}}(\partial \phi)(\tau) \\
\|\partial \Omega \phi\|_{L^{2}\left(\Sigma_{t}\right)} & \lesssim\|\partial \Omega \phi\|_{L^{2}\left(\Sigma_{t_{0}}\right)}+\lambda^{-\epsilon} \sup _{\tau \in\left[t_{0}, t_{*}\right]} E^{\frac{1}{2}}(\partial \phi)(\tau)
\end{aligned}
$$

First we prove some preliminary results:

\subsection{Commutation results}

Here we will record the commutation formula between a vectorfield $X$ and the D'Alembertian $\square_{g}$. This result will be used crucially in this section. We start first with

Lemma 8.2 ([6]) For a vectorfield $X$ with the deformation tensor ${ }^{X} \pi$ and a 1-form $V$ on a space-time manifold with metric $g$ and corresponding connection $\mathcal{D}$, we have

$$
\mathcal{D}_{\sigma}\left(\mathcal{L}_{X} V_{\alpha}\right)=\mathcal{L}_{X}\left(\mathcal{D}_{\sigma} V_{\alpha}\right)+{ }^{X} \Gamma_{\alpha \sigma \lambda} V^{\lambda}
$$


where

$$
{ }^{X} \Gamma_{\alpha \sigma \lambda}=\frac{1}{2}\left(\mathcal{D}_{\alpha}{ }^{X} \pi_{\sigma \lambda}+\mathcal{D}_{\sigma}{ }^{X} \pi_{\alpha \lambda}-\mathcal{D}_{\lambda}{ }^{X} \pi_{\alpha \sigma}\right) .
$$

Proof The formula for the Lie derivative gives us :

$$
\mathcal{L}_{X} V_{\alpha}=X^{\gamma} \cdot \mathcal{D}_{\gamma} V_{\alpha}+V_{\gamma} \cdot \mathcal{D}_{\alpha} X^{\gamma}
$$

This implies:

$$
\mathcal{D}_{\sigma}\left(\mathcal{L}_{X} V_{\alpha}\right)=X^{\gamma} \cdot \mathcal{D}_{\sigma} \mathcal{D}_{\gamma} V_{\alpha}+\mathcal{D}_{\sigma} X^{\gamma} \cdot \mathcal{D}_{\gamma} V_{\alpha}+\mathcal{D}_{\sigma} V_{\gamma} \cdot \mathcal{D}_{\alpha} X^{\gamma}+V_{\gamma} \cdot \mathcal{D}_{\sigma} \mathcal{D}_{\alpha} X^{\gamma}
$$

On the other hand:

$$
\mathcal{L}_{X}\left(\mathcal{D}_{\sigma} V_{\alpha}\right)=X^{\gamma} \cdot \mathcal{D}_{\gamma} \mathcal{D}_{\sigma} V_{\alpha}+\mathcal{D}_{\sigma} X^{\gamma} \cdot \mathcal{D}_{\gamma} V_{\alpha}+\mathcal{D}_{\sigma} V_{\gamma} \cdot \mathcal{D}_{\alpha} X^{\gamma}
$$

Subtracting term by term these relations, we end up with:

$$
\begin{aligned}
\mathcal{D}_{\sigma}\left(\mathcal{L}_{X} V_{\alpha}\right)-\mathcal{L}_{X}\left(\mathcal{D}_{\sigma} V_{\alpha}\right) & =X^{\gamma}\left(\mathcal{D}_{\sigma} \mathcal{D}_{\gamma} V_{\alpha}-\mathcal{D}_{\gamma} \mathcal{D}_{\sigma} V_{\alpha}\right)+V_{\gamma} \cdot \mathcal{D}_{\sigma} \mathcal{D}_{\alpha} X^{\gamma} \\
& =R_{\alpha \mu \sigma \lambda} V^{\mu} X^{\lambda}+V_{\gamma} \cdot \mathcal{D}_{\sigma} \mathcal{D}_{\alpha} X^{\gamma}
\end{aligned}
$$

We investigate the ${ }^{X} \Gamma_{\alpha \sigma \lambda}$ term, which we can detail as

$$
\begin{aligned}
{ }^{X} \Gamma_{\alpha \sigma \lambda} & =\frac{1}{2}\left(\mathcal{D}_{\alpha} \mathcal{D}_{\sigma} X_{\lambda}+\mathcal{D}_{\alpha} \mathcal{D}_{\lambda} X_{\sigma}+\mathcal{D}_{\sigma} \mathcal{D}_{\alpha} X_{\lambda}+\mathcal{D}_{\sigma} \mathcal{D}_{\lambda} X_{\alpha}-\mathcal{D}_{\lambda} \mathcal{D}_{\alpha} X_{\sigma}-\mathcal{D}_{\lambda} \mathcal{D}_{\sigma} X_{\alpha}\right) \\
& =\frac{1}{2}\left(\mathcal{D}_{\alpha} \mathcal{D}_{\sigma} X_{\lambda}+\mathcal{D}_{\sigma} \mathcal{D}_{\alpha} X_{\lambda}+R_{\sigma \mu \alpha \lambda} X^{\mu}+R_{\alpha \mu \sigma \lambda} X^{\mu}\right)=\mathcal{D}_{\sigma} \mathcal{D}_{\alpha} X_{\lambda}+R_{\alpha \lambda \sigma \mu} X^{\mu}
\end{aligned}
$$

This, of course, concludes the proof of the lemma. We are now ready to state and prove our main commutation result:

Proposition 8.3 ([6]) For an arbitrary vectorfield $X$ with the deformation tensor ${ }^{X} \pi$, trace

$$
\operatorname{tr}^{X} \pi=g^{\alpha \beta X} \pi_{\alpha \beta}
$$

and traceless part

$$
{ }^{X} \hat{\pi}_{\alpha \beta}={ }^{X} \pi_{\alpha \beta}-\frac{1}{n+1} \operatorname{tr}^{X} \pi g_{\alpha \beta},
$$

we have the following commutation formula:

$$
\begin{aligned}
& \square_{g}(X \phi)-X\left(\square_{g} \phi\right)={ }^{X} \pi^{\alpha \beta} \cdot \mathcal{D}_{\alpha} \mathcal{D}_{\beta} \phi+\mathcal{D}^{\alpha X} \pi_{\alpha \lambda} \cdot \mathcal{D}^{\lambda} \phi-\frac{1}{2} \mathcal{D}^{\lambda} \operatorname{tr}{ }^{X} \pi \cdot \mathcal{D}_{\lambda} \phi \\
& { }^{X} \hat{\pi}^{\alpha \beta} \cdot \mathcal{D}_{\alpha} \mathcal{D}_{\beta} \phi+\mathcal{D}^{\alpha}{ }^{X} \hat{\pi}_{\alpha \lambda} \cdot \mathcal{D}^{\lambda} \phi+\left(\frac{1}{n+1}-\frac{1}{2}\right) \mathcal{D}^{\lambda} t r^{X} \pi \cdot \mathcal{D}_{\lambda} \phi+\frac{1}{n+1} t r{ }^{X} \pi \cdot \square_{g} \phi
\end{aligned}
$$


Proof Using the decomposition $\square_{g}=g^{\alpha \beta} \mathcal{D}_{\alpha} \mathcal{D}_{\beta}$, we can infer that:

$$
\begin{aligned}
& \square_{g}(X \phi)-X\left(\square_{g} \phi\right)=g^{\alpha \beta} \mathcal{D}_{\alpha} \mathcal{D}_{\beta}\left(\mathcal{D}_{X} \phi\right)-\mathcal{L}_{X}\left(g^{\alpha \beta} \mathcal{D}_{\alpha} \mathcal{D}_{\beta} \phi\right)=g^{\alpha \beta} \mathcal{D}_{\alpha}\left(\mathcal{D}_{X} \mathcal{D}_{\beta} \phi+\right. \\
& \left.+\mathcal{D}_{\beta} X^{\gamma} \cdot \mathcal{D}_{\gamma} \phi\right)-\mathcal{L}_{X}\left(g^{\alpha \beta}\right) \mathcal{D}_{\alpha} \mathcal{D}_{\beta} \phi-g^{\alpha \beta} \mathcal{L}_{X}\left(\mathcal{D}_{\alpha} \mathcal{D}_{\beta} \phi\right)=g^{\alpha \beta}\left(\mathcal{D}_{\alpha} \mathcal{L}_{X} \mathcal{D}_{\beta} \phi-\right. \\
& \left.-\mathcal{L}_{X} \mathcal{D}_{\alpha} \mathcal{D}_{\beta} \phi\right)+\left(g^{\alpha \gamma} \cdot \mathcal{D}_{\gamma} X^{\beta}+g^{\beta \gamma} \cdot \mathcal{D}_{\gamma} X^{\alpha}\right) \mathcal{D}_{\alpha} \mathcal{D}_{\beta} \phi
\end{aligned}
$$

Here we take advantage of (154) to conclude that:

$$
\begin{aligned}
& \square_{g}(X \phi)-X\left(\square_{g} \phi\right)=g^{\alpha \beta} \cdot \frac{1}{2}\left(\mathcal{D}_{\alpha}{ }^{X} \pi_{\beta \lambda}+\mathcal{D}_{\beta}{ }^{X} \pi_{\alpha \lambda}-\mathcal{D}_{\lambda}{ }^{X} \pi_{\alpha \beta}\right) \mathcal{D}^{\lambda} \phi+ \\
& +{ }^{X} \pi^{\alpha \beta} \cdot \mathcal{D}_{\alpha} \mathcal{D}_{\beta} \phi=\mathcal{D}^{\alpha X} \pi_{\alpha \lambda} \cdot \mathcal{D}^{\lambda} \phi-\frac{1}{2} \mathcal{D}^{\lambda} t r^{X} \pi \cdot \mathcal{D}_{\lambda} \phi+{ }^{X} \pi^{\alpha \beta} \cdot \mathcal{D}_{\alpha} \mathcal{D}_{\beta} \phi,
\end{aligned}
$$

which ends the proof. An immediate consequence of this result is the following:

Lemma 8.4 Under the assumptions of Theorem 5.1:

$$
\begin{aligned}
& \square_{g}(\Omega \phi)={ }^{\Omega} \pi^{\alpha \beta} \cdot \mathcal{D}_{\alpha} \mathcal{D}_{\beta} \phi+\mathcal{D}^{\alpha}{ }^{\Omega} \pi_{\alpha \lambda} \cdot \mathcal{D}^{\lambda} \phi-\frac{1}{2} \mathcal{D}^{\lambda} t r^{\Omega} \pi \cdot \mathcal{D}_{\lambda} \phi \\
& \square_{g}(S \phi)={ }^{S} \hat{\pi}^{\alpha \beta} \cdot \mathcal{D}_{\alpha} \mathcal{D}_{\beta} \phi+\mathcal{D}^{\alpha S} \hat{\pi}_{\alpha \lambda} \cdot \mathcal{D}^{\lambda} \phi-\frac{1}{6} \mathcal{D}^{\lambda} t r{ }^{S} \pi \cdot \mathcal{D}_{\lambda} \phi
\end{aligned}
$$

\subsection{Proof of estimate (153)}

We start first with:

Proposition 8.5 The deformation tensor ${ }^{\Omega} \pi$ of the angular momentum vectorfield

$$
\Omega=s \cdot A
$$

satisfies

$$
\begin{aligned}
& { }^{\Omega} \pi_{L L}=0 \\
& { }^{\Omega} \pi_{\underline{L L}}=4 s\left(\eta-\kappa_{A N}\right) \\
& { }^{\Omega} \pi_{A A}=0 \\
& { }^{\Omega} \pi_{L \underline{L}}=-2 s\left(\eta-\kappa_{A N}\right) \\
& { }^{\Omega} \pi_{L A}=-s \chi+1 \\
& { }^{\Omega} \pi_{\underline{L A}}=-s \underline{\chi}+1-2 b^{-1} \\
& \operatorname{tr}^{\Omega} \pi=-{ }^{\Omega} \pi_{L \underline{L}}+{ }^{\Omega} \pi_{A A}=2 s\left(\eta-\kappa_{A N}\right)
\end{aligned}
$$

Proof Using the expression for $\Omega$, we can infer that:

$$
{ }^{\Omega} \pi_{\alpha \beta}=s\left(<\mathcal{D}_{\alpha} A, \partial_{\beta}>+<\mathcal{D}_{\beta} A, \partial_{\alpha}>\right)+\partial_{\alpha} s<A, \partial_{\beta}>+\partial_{\beta} s<A, \partial_{\alpha}>
$$


This implies:

$$
\begin{aligned}
& { }^{\Omega} \pi_{L L}=2 s<\mathcal{D}_{L} A, L>+2 L(s)<A, L>=0 \\
& { }^{\Omega} \pi_{\underline{L L}}=2 s<\mathcal{D}_{\underline{L}} A, \underline{L}>+2 \underline{L}(s)<A, \underline{L}>=2 s(-2 \underline{\xi})=4 s\left(\eta-\kappa_{A N}\right) \\
& { }^{\Omega} \pi_{A A}=2 s<\mathcal{D}_{A} A, A>+2 A(s)<A, A>=0 \\
& { }^{\Omega} \pi_{L A}=s\left(<\mathcal{D}_{L} A, A>+<\mathcal{D}_{A} A, L>\right)+L(s)<A, A>+A(s)<A, L>= \\
& =-s \chi+1 \\
& { }^{\Omega} \pi_{\underline{L A}}=s\left(<\mathcal{D}_{\underline{L}} A, A>+<\mathcal{D}_{A} A, \underline{L}>\right)+\underline{L}(s)<A, A>+A(s)<A, \underline{L}>= \\
& =-s \underline{\chi}+1-2 b^{-1} \\
& { }^{\Omega} \pi_{L \underline{L}}=s\left(<\mathcal{D}_{L} A, \underline{L}>+<\mathcal{D}_{\underline{L}} A, L>\right)+L(s)<A, \underline{L}>+\underline{L}(s)<A, L>= \\
& =s(-2 \underline{\eta}-2 \eta)=-2 s\left(\eta-\kappa_{A N}\right) .
\end{aligned}
$$

Using the energy estimate (125) applied to equation (156), we obtain:

$$
\begin{aligned}
\|\partial \Omega \phi\|_{L^{2}\left(\Sigma_{t}\right)} & \lesssim\|\partial \Omega \phi\|_{L^{2}\left(\Sigma_{t_{0}}\right)}+ \\
& +\int_{t_{0}}^{t}\left\|^{\Omega} \pi^{\alpha \beta} \cdot \mathcal{D}_{\alpha} \mathcal{D}_{\beta} \phi+\mathcal{D}^{\alpha} \pi_{\alpha \lambda} \cdot \mathcal{D}^{\lambda} \phi-\frac{1}{2} \mathcal{D}^{\lambda} t r{ }^{\Omega} \cdot \mathcal{D}_{\lambda} \phi\right\|_{L^{2}\left(\Sigma_{\tau}\right)} d \tau
\end{aligned}
$$

Detailing the first integral term, we infer that:

$$
\begin{aligned}
\int_{t_{0}}^{t}\left\|^{\Omega} \pi^{\alpha \beta} \mathcal{D}_{\alpha} \mathcal{D}_{\beta} \phi\right\|_{L^{2}\left(\Sigma_{\tau}\right)} d \tau \lesssim \int_{t_{0}}^{t}\left(\left\|^{\Omega} \pi_{L L} \mathcal{D}_{\underline{L}} \mathcal{D}_{\underline{L}} \phi\right\|_{L^{2}\left(\Sigma_{\tau}\right)}+\left\|^{\Omega} \pi_{\underline{L L}} \mathcal{D}_{L} \mathcal{D}_{L} \phi\right\|_{L^{2}\left(\Sigma_{\tau}\right)}+\right. \\
+\left\|^{\Omega} \pi_{A A} \mathcal{D}_{A} \mathcal{D}_{A} \phi\right\|_{L^{2}\left(\Sigma_{\tau}\right)}+\left\|^{\Omega} \pi_{L A} \mathcal{D}_{A} \mathcal{D}_{\underline{L}} \phi\right\|_{L^{2}\left(\Sigma_{\tau}\right)}+\left\|^{\Omega} \pi_{\underline{L A}} \mathcal{D}_{L} \mathcal{D}_{A} \phi\right\|_{L^{2}\left(\Sigma_{\tau}\right)}+ \\
\left.\quad+\left\|^{\Omega} \pi_{L \underline{L}} \mathcal{D}_{L} \mathcal{D}_{\underline{L}} \phi\right\|_{L^{2}\left(\Sigma_{\tau}\right)}\right) d \tau
\end{aligned}
$$

The first and the third terms of the right hand side vanishes due to the formulae in Proposition 8.5. Investigating one by one the remaining terms we obtain:

$$
\begin{aligned}
\int_{t_{0}}^{t}\left\|^{\Omega} \pi_{\underline{L L}} \mathcal{D}_{L} \mathcal{D}_{L} \phi\right\|_{L^{2}\left(\Sigma_{\tau}\right)} d \tau & \lesssim \int_{t_{0}}^{t}\left\|s\left(\eta-\kappa_{A N}\right)\left(L(L \phi)+\kappa_{N N} L \phi\right)\right\|_{L^{2}\left(\Sigma_{\tau}\right)} d \tau \lesssim \\
& \lesssim \int_{t_{0}}^{t}\left(1+\lambda^{-\bar{a}} \tau\right)\left(\tau^{\frac{1}{2}} \lambda^{-\bar{a}-\frac{1-a}{2}+\epsilon}+\lambda^{-\bar{a}}\right) E^{\frac{1}{2}}(\partial \phi)(\tau) d \tau \lesssim \\
& \lesssim \lambda^{-\epsilon} \sup _{\tau \in\left[t_{0}, t_{*}\right]} E^{\frac{1}{2}}(\partial \phi)(\tau) \\
\int_{t_{0}}^{t}\left\|^{\Omega} \pi_{L A} \mathcal{D}_{A} \mathcal{D}_{\underline{L}} \phi\right\|_{L^{2}\left(\Sigma_{\tau}\right)} d \tau & \lesssim \int_{t_{0}}^{t}\left\|s\left(\chi-\frac{1}{s}\right)\left(A(\underline{L} \phi)-\underline{\chi} A \phi-\kappa_{A N} \underline{L} \phi\right)\right\|_{L^{2}\left(\Sigma_{\tau}\right)} d \tau \\
& \lesssim \int_{t_{0}}^{t}\left(\tau \lambda^{-2 \bar{a}}+\lambda^{-\bar{a}}\right) E^{\frac{1}{2}}(\partial \phi)(\tau) d \tau \\
& \lesssim \lambda^{-\epsilon} \sup _{\tau \in\left[t_{0}, t_{*}\right]} E^{\frac{1}{2}}(\partial \phi)(\tau)
\end{aligned}
$$




$$
\begin{aligned}
\int_{t_{0}}^{t}\left\|^{\Omega} \pi_{\underline{L} A} \mathcal{D}_{L} \mathcal{D}_{A} \phi\right\|_{L^{2}\left(\Sigma_{\tau}\right)} d \tau & \lesssim \int_{t_{0}}^{t}\left\|\left(-s \underline{\chi}+1-2 b^{-1}\right)\left(L(A \phi)+\kappa_{A N} L \phi\right)\right\|_{L^{2}\left(\Sigma_{\tau}\right)} d \tau \\
& \lesssim \int_{t_{0}}^{t}\left(\tau \lambda^{-2 \bar{a}}+\lambda^{-\bar{a}}\right) E^{\frac{1}{2}}(\partial \phi)(\tau) d \tau \\
& \lesssim \lambda^{-\epsilon} \sup _{\tau \in\left[t_{0}, t_{*}\right]} E^{\frac{1}{2}}(\partial \phi)(\tau) \\
\int_{t_{0}}^{t}\left\|^{\Omega} \pi_{L \underline{L}} \mathcal{D}_{L} \mathcal{D}_{\underline{L}} \phi\right\|_{L^{2}\left(\Sigma_{\tau}\right)} d \tau & \lesssim \int_{t_{0}}^{t}\left\|s\left(\eta-\kappa_{A N}\right)\left(L(\underline{L} \phi)-\kappa_{N N} \underline{L} \phi+2 \kappa_{A N} A \phi\right)\right\|_{L^{2}\left(\Sigma_{\tau}\right)} d \tau \\
& \lesssim \int_{t_{0}}^{t}\left(1+\lambda^{-\bar{a}} \tau\right)\left(\tau^{\frac{1}{2}} \lambda^{-\bar{a}-\frac{1-a}{2}+\epsilon}+\lambda^{-\bar{a}}\right) E^{\frac{1}{2}}(\partial \phi)(\tau) d \tau \\
& \lesssim \lambda^{-\epsilon} \sup _{\tau \in\left[t_{0}, t_{*}\right]} E^{\frac{1}{2}}(\partial \phi)(\tau)
\end{aligned}
$$

The other two terms that we encounter in the commutation formula (156) will be considered together. Lowering the upper indices we obtain:

$$
\begin{aligned}
& \mathcal{D}^{\alpha} \pi_{\alpha \beta} \cdot \mathcal{D}^{\beta} \phi-\frac{1}{2} \mathcal{D}^{\alpha}\left(\operatorname{tr}^{\Omega} \pi\right) \cdot \mathcal{D}_{\alpha} \phi= \\
& =\left(\frac{1}{4} \mathcal{D}_{\underline{L}}{ }^{\Omega} \pi_{L \underline{L}}+\frac{1}{4} \mathcal{D}_{L}{ }^{\Omega} \pi_{\underline{L L}}-\frac{1}{2} \mathcal{D}_{A}{ }^{\Omega} \pi_{A \underline{L}}+\frac{1}{4} \underline{L}\left(t^{\Omega} \pi\right)\right) \cdot L \phi+ \\
& +\left(\frac{1}{4} \mathcal{D}_{\underline{L}}{ }^{\Omega} \pi_{L L}+\frac{1}{4} \mathcal{D}_{L}{ }^{\Omega} \pi_{\underline{L L}}-\frac{1}{2} \mathcal{D}_{A}{ }^{\Omega} \pi_{A L}+\frac{1}{4} L\left(t r^{\Omega} \pi\right)\right) \cdot \underline{L} \phi+ \\
& +\left(-\frac{1}{2} \mathcal{D}_{\underline{L}}{ }^{\Omega} \pi_{L A}-\frac{1}{2} \mathcal{D}_{L}{ }^{\Omega} \pi_{\underline{L A}}+\mathcal{D}_{A}{ }^{\Omega} \pi_{A A}-\frac{1}{2} A\left(t^{\Omega} \pi\right)\right) \cdot A \phi
\end{aligned}
$$

Detailing each parenthesis, we can infer that:

$$
\begin{aligned}
& \frac{1}{4} \mathcal{D}_{\underline{L}}{ }^{\Omega} \pi_{L \underline{L}}+\frac{1}{4} \mathcal{D}_{L}{ }^{\Omega} \pi_{\underline{L L}}-\frac{1}{2} \mathcal{D}_{A}{ }^{\Omega} \pi_{A \underline{L}}+\frac{1}{4} \underline{L}\left(\operatorname{tr}^{\Omega} \pi\right)=\frac{1}{4}\left(\underline{L}\left({ }^{\Omega} \pi_{L \underline{L}}\right)-2 \eta^{\Omega} \pi_{A \underline{L}}-\right. \\
& \left.-\kappa_{N N}{ }^{\Omega} \pi_{L \underline{L}}-2 \underline{\xi}^{\Omega} \pi_{A L}+\kappa_{N N}{ }^{\Omega} \pi_{L \underline{L}}\right)+\frac{1}{4}\left(L\left({ }^{\Omega} \pi_{\underline{L L}}\right)+4 \kappa_{A N}{ }^{\Omega} \pi_{A \underline{L}}-2 \kappa_{N N}{ }^{\Omega} \pi_{\underline{L L}}\right)- \\
& -\frac{1}{2}\left(A\left({ }^{\Omega} \pi_{A \underline{L}}\right)-\frac{1}{2} \chi^{\Omega} \pi_{\underline{L L}}-\frac{1}{2} \underline{\chi}^{\Omega} \pi_{L \underline{L}}-\underline{\chi}^{\Omega} \pi_{A A}-\kappa_{A N}{ }^{\Omega} \pi_{A \underline{L}}\right)+\frac{1}{4} \underline{L}\left(-{ }^{\Omega} \pi_{L \underline{L}}\right)
\end{aligned}
$$

Remark We notice here the cancellation of $\frac{1}{4} \underline{L}\left({ }^{\Omega} \pi_{L \underline{L}}\right)$ with the last term $\frac{1}{4} \underline{L}\left(-{ }^{\Omega} \pi_{L \underline{L}}\right)$. These

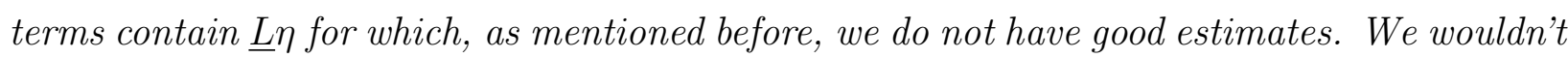
have been able to prove our result, unless this cancellation took place.

$$
\begin{aligned}
& \frac{1}{4} \mathcal{D}_{\underline{L}}{ }^{\Omega} \pi_{L L}+\frac{1}{4} \mathcal{D}_{L}{ }^{\Omega} \pi_{\underline{L} L}-\frac{1}{2} \mathcal{D}_{A}{ }^{\Omega} \pi_{A L}+\frac{1}{4} L\left(t r^{\Omega} \pi\right)=\frac{1}{4}\left(\underline{L}\left({ }^{\Omega} \pi_{L L}\right)-4 \eta^{\Omega} \pi_{A L}-\right. \\
& \left.-2 \kappa_{N N}{ }^{\Omega} \pi_{L L}\right)+\frac{1}{4}\left(L\left({ }^{\Omega} \pi_{\underline{L} L}\right)+2 \kappa_{A N}{ }^{\Omega} \pi_{A L}-\kappa_{N N}{ }^{\Omega} \pi_{L \underline{L}}+\kappa_{N N}{ }^{\Omega} \pi_{L \underline{L}}\right)-\frac{1}{2}\left(A\left({ }^{\Omega} \pi_{A L}\right)-\right. \\
& \left.-\frac{1}{2} \chi^{\Omega} \pi_{L \underline{L}}-\frac{1}{2} \underline{\chi}^{\Omega} \pi_{L L}-\chi^{\Omega} \pi_{A A}+\kappa_{A N}{ }^{\Omega} \pi_{A L}\right)+\frac{1}{4} L\left(-{ }^{\Omega} \pi_{\underline{L L}}\right)
\end{aligned}
$$




$$
\begin{aligned}
& -\frac{1}{2} \mathcal{D}_{\underline{L}}{ }^{\Omega} \pi_{L A}-\frac{1}{2} \mathcal{D}_{L}{ }^{\Omega} \pi_{\underline{L A}}+\mathcal{D}_{A}{ }^{\Omega} \pi_{A A}-\frac{1}{2} A\left(\operatorname{tr}^{\Omega} \pi\right)=-\frac{1}{2}\left(\underline{L}\left({ }^{\Omega} \pi_{L A}\right)-2 \eta^{\Omega} \pi_{A A}-\right. \\
& \left.-\kappa_{N N}{ }^{\Omega} \pi_{A L}-\eta^{\Omega} \pi_{\underline{L} L}-\underline{\xi}^{\Omega} \pi_{L L}\right)-\frac{1}{2}\left(L\left({ }^{\Omega} \pi_{\underline{L A}}\right)+2 \kappa_{A N}{ }^{\Omega} \pi_{A A}-\kappa_{N N}{ }^{\Omega} \pi_{A \underline{L}}+\right. \\
& \left.+\kappa_{A N}{ }^{\Omega} \pi_{\underline{L} L}\right)+\left(A\left({ }^{\Omega} \pi_{A A}\right)-\underline{\chi}^{\Omega} \pi_{L A}-\chi^{\Omega} \pi_{\underline{L A}}\right)+\frac{1}{2} A\left({ }^{\Omega} \pi_{\underline{L} L}\right)
\end{aligned}
$$

Taking into account the explicit formulae for the different components of the deformation tensor provided in Proposition 8.5, we can reduce the above expressions to

$$
\begin{aligned}
& \frac{1}{4} \mathcal{D}_{\underline{L}}{ }^{\Omega} \pi_{L \underline{L}}+\frac{1}{4} \mathcal{D}_{L} \Omega_{\underline{L L}}-\frac{1}{2} \mathcal{D}_{A}{ }^{\Omega} \pi_{A \underline{L}}+\frac{1}{4} \underline{L}\left(t^{\Omega} \pi\right)=L\left(s\left(\eta-\kappa_{A N}\right)\right)- \\
& -\frac{1}{2} A\left(-s \underline{\chi}+1-2 b^{-1}\right)-\frac{1}{2}\left(\eta+\kappa_{A N}\right)\left(-s \underline{\chi}+1-2 b^{-1}\right)+ \\
+ & \left(\eta-\kappa_{A N}\right)\left(\frac{1}{2}+s \chi+s \kappa_{A A}-s \kappa_{N N}\right) \\
& \frac{1}{4} \mathcal{D}_{\underline{L}}{ }^{\Omega} \pi_{L L}+\frac{1}{4} \mathcal{D}_{L}{ }^{\Omega} \pi_{\underline{L} L}-\frac{1}{2} \mathcal{D}_{A}{ }^{\Omega} \pi_{A L}+\frac{1}{4} L\left(r^{\Omega} \pi\right)=-\frac{1}{2} A(-s \chi+1)- \\
& -\eta(-s \chi+1)-\frac{1}{2} s \chi\left(\eta-\kappa_{A N}\right) \\
- & \frac{1}{2} \mathcal{D}_{\underline{L}}{ }^{\Omega} \pi_{L A}-\frac{1}{2} \mathcal{D}_{L}{ }^{\Omega} \pi_{\underline{L A}}+\mathcal{D}_{A} \Omega_{\pi_{A A}}-\frac{1}{2} A\left(t r^{\Omega} \pi\right)=-\frac{1}{2} \underline{L}(-s \chi+1)- \\
- & \frac{1}{2} L\left(-s \underline{\chi}+1-2 b^{-1}\right)-A\left(s\left(\eta-\kappa_{A N}\right)\right)+\left(\frac{1}{2} \kappa_{N N}-\chi\right)\left(-s \underline{\chi}+1-2 b^{-1}\right)+ \\
+ & \left(\frac{1}{2} \kappa_{N N}-\underline{\chi}\right)(-s \chi+1)-s\left(\eta-\kappa_{A N}\right)^{2}
\end{aligned}
$$

All the terms in these expressions which do not have a derivative with respect to the null frame $\{L, \underline{L}, A\}$ and which we denote generically by $X$, can be estimated in the $L^{\infty}$-norm as follows

$$
|X| \lesssim\left(1+\lambda^{-\bar{a}} \tau\right)\left(\tau^{\frac{1}{2}} \lambda^{-\bar{a}-\frac{1-a}{2}+\epsilon}+\lambda^{-\bar{a}}\right)
$$

Then, the integral expressions corresponding to these terms satisfy:

$$
\begin{aligned}
\int_{t_{0}}^{t}\|X \partial \phi\|_{L^{2}\left(\Sigma_{\tau}\right)} d \tau & \lesssim \int_{t_{0}}^{t}\left(1+\lambda^{-\bar{a}} \tau\right)\left(\tau^{\frac{1}{2}} \lambda^{-\bar{a}-\frac{1-a}{2}+\epsilon}+\lambda^{-\bar{a}}\right) E^{\frac{1}{2}}(\partial \phi)(\tau) d \tau \\
& \lesssim \lambda^{-\epsilon} \sup _{\tau \in\left[t_{0}, t_{*}\right]} E^{\frac{1}{2}}(\partial \phi)(\tau)
\end{aligned}
$$

We denote the terms which involve derivatives by $Y$. For their corresponding integrals we split the discussion into the interior part, where we will use a similar argument as in the case of Morawetz vectorfield $K$, and the exterior part, where we will take advantage of the integral estimate (82):

$$
\int_{t_{0}}^{t}\|Y \partial \phi\|_{L^{2}\left(\Sigma_{\tau}\right)} d \tau \lesssim \int_{t_{0}}^{t}\|Y \partial \phi \omega\|_{L^{2}\left(\Sigma_{\tau}\right)} d \tau+\int_{t_{0}}^{t}\|Y \partial \phi(1-\omega)\|_{L^{2}\left(\Sigma_{\tau}\right)} d \tau \lesssim
$$




$$
\begin{aligned}
& \lesssim \int_{t_{0}}^{t} \tau^{-\frac{1}{2}} \sup _{0 \leq u \leq \frac{\tau}{2}}\|Y\|_{L^{2}\left(S_{\tau, u}\right)} E^{\frac{1}{2}}(\partial \phi)(\tau) d \tau+\int_{t_{0}}^{t} \tau^{-1}\|Y(1-\omega)\|_{L^{2}\left(\Sigma_{\tau}\right)} E^{\frac{1}{2}}(\partial \phi)(\tau) d \tau \\
& \lesssim \int_{t_{0}}^{t} \tau^{-\frac{1}{2}} \sup _{0 \leq u \leq \frac{\tau}{2}}\|Y\|_{L^{2}\left(S_{\tau, u}\right)} E^{\frac{1}{2}}(\partial \phi)(\tau) d \tau+\int_{t_{0}}^{t} \tau^{-\frac{1}{2}} \sup _{\frac{\tau}{2} \leq u \leq \tau}\|Y\|_{L^{2}\left(S_{\tau, u}\right)} E^{\frac{1}{2}}(\partial \phi)(\tau) d \tau
\end{aligned}
$$

A typical " $Y$ "-term is, for example, $L\left(s\left(\eta-\kappa_{A N}\right)\right)$. Using the asymptotic estimates (85), we can evaluate it as follows:

$$
\begin{aligned}
\left\|L\left(s\left(\eta-\kappa_{A N}\right)\right)\right\|_{L^{2}\left(S_{\tau, u}\right)} & \lesssim s\left\|L\left(\eta-\kappa_{A N}\right)\right\|_{L^{2}\left(S_{\tau, u}\right)}+\left\|\eta-\kappa_{A N}\right\|_{L^{2}\left(S_{\tau, u}\right)} \\
& \lesssim s\left(\lambda^{-\bar{a}-\frac{1-a}{2}+\epsilon}+s^{-\frac{1}{2}} \lambda^{-\bar{a}}\right)+s^{\frac{1}{2}}\left(s^{\frac{1}{2}} \lambda^{-\bar{a}-\frac{1-a}{2}+\epsilon}+\lambda^{-\bar{a}}\right) \\
& \lesssim s \lambda^{-\bar{a}-\frac{1-a}{2}+\epsilon}+s^{\frac{1}{2}} \lambda^{-\bar{a}}
\end{aligned}
$$

Hence, the general estimate for $Y$ is:

$$
\sup _{0 \leq u \leq \tau}\|Y\|_{L^{2}\left(S_{\tau, u}\right)} \lesssim \tau \lambda^{-\bar{a}-\frac{1-a}{2}+\epsilon}+\tau^{\frac{1}{2}} \lambda^{-\bar{a}}
$$

Therefore the integral above can be evaluated by:

$$
\begin{aligned}
\int_{t_{0}}^{t}\|Y \partial \phi\|_{L^{2}\left(\Sigma_{\tau}\right)} d \tau & \lesssim \int_{t_{0}}^{t}\left(\tau^{\frac{1}{2}} \lambda^{-\bar{a}-\frac{1-a}{2}+\epsilon}+\lambda^{-\bar{a}}\right) E^{\frac{1}{2}}(\partial \phi)(\tau) d \tau \\
& \lesssim \lambda^{-\epsilon} \sup _{\tau \in\left[t_{0}, t_{*}\right]} E^{\frac{1}{2}}(\partial \phi)(\tau)
\end{aligned}
$$

This concludes the proof of estimate (153).

\subsection{Proof of estimate (152)}

As in the previous section, we start with:

Proposition 8.6 The deformation tensor ${ }^{S} \pi$ of the scaling vectorfield

$$
S=\frac{1}{2}(u \underline{L}+\underline{u} L)
$$

satisfies

$$
\begin{aligned}
& { }^{S} \pi_{L L}=-2 u \kappa_{N N} \\
& { }^{S} \pi_{\underline{L L}}=4\left(b^{-1}-1\right)-2 \underline{u} \kappa_{N N} \\
& { }^{S} \pi_{A A}=u \underline{\chi}+\underline{u} \chi \\
& { }^{S} \pi_{L \underline{L}}=-2\left(1+b^{-1}\right)+(u+\underline{u}) \kappa_{N N} \\
& { }^{S} \pi_{L A}=-2 u \kappa_{A N} \\
& { }^{S} \pi_{\underline{L A}}=u \underline{\xi}+\underline{u}\left(\eta+\kappa_{A N}\right) \\
& { }_{t}{ }^{S} \pi=-{ }^{S} \pi_{L \underline{L}}+{ }^{S} \pi_{A A}=u \underline{\chi}+\underline{u} \chi-(u+\underline{u}) \kappa_{N N}+2\left(1+b^{-1}\right)
\end{aligned}
$$


Proof Using the above formula for $S$, we infer that:

$$
\begin{aligned}
{ }^{S} \pi_{\alpha \beta} & =\frac{1}{2} u\left(<\mathcal{D}_{\alpha} \underline{L}, \partial_{\beta}>+<\mathcal{D}_{\beta} \underline{L}, \partial_{\alpha}>\right)+\frac{1}{2} \underline{u}\left(<\mathcal{D}_{\alpha} L, \partial_{\beta}>+<\mathcal{D}_{\beta} L, \partial_{\alpha}>\right)+ \\
& +\frac{1}{2} \partial_{\alpha} u<\underline{L}, \partial_{\beta}>+\frac{1}{2} \partial_{\beta} u<\underline{L}, \partial_{\alpha}>+\frac{1}{2} \partial_{\alpha} \underline{u}<L, \partial_{\beta}>+\frac{1}{2} \partial_{\beta} \underline{u}<L, \partial_{\alpha}>
\end{aligned}
$$

This implies

$$
\begin{aligned}
& S_{\pi_{L L}}=u<\mathcal{D}_{L} \underline{L}, L>+\underline{u}<\mathcal{D}_{L} L, L>+L(u)<\underline{L}, L>+L(\underline{u})<L, L>= \\
& =-2 u \kappa_{N N} \\
& S_{\pi_{\underline{L}}}=u<\mathcal{D}_{\underline{L}} \underline{L}, \underline{L}>+\underline{u}<\mathcal{D}_{\underline{L}} L, \underline{L}>+\underline{L}(u)<\underline{L}, L>+\underline{L}(\underline{u})<L, L>= \\
& =\underline{u}\left(-2 \kappa_{N N}\right)+(-2)\left(2-2 b^{-1}\right)=4\left(b^{-1}-1\right)-2 \underline{u} \kappa_{N N} \\
& { }^{S} \pi_{A A}=u<\mathcal{D}_{A} \underline{L}, A>+\underline{u}<\mathcal{D}_{A} L, A>+A(u)<\underline{L}, A>+A(\underline{u})<L, A>= \\
& =u \underline{\chi}+\underline{u} \chi \\
& S_{\pi_{L A}}=\frac{1}{2} u\left(<\mathcal{D}_{L} \underline{L}, A>+<\mathcal{D}_{A} \underline{L}, L>\right)+\frac{1}{2} \underline{u}\left(<\mathcal{D}_{L} L, A>+<\mathcal{D}_{A} L, L>\right)+ \\
& +\frac{1}{2} L(u)<\underline{L}, A>+\frac{1}{2} A(u)<\underline{L}, L>+\frac{1}{2} L(\underline{u})<L, A>+\frac{1}{2} A(\underline{u})<L, L>= \\
& =\frac{1}{2} u\left(2 \underline{\eta}-2 \kappa_{A N}\right)=-2 u \kappa_{A N} \\
& S_{\pi_{\underline{L} A}}=\frac{1}{2} u\left(<\mathcal{D}_{\underline{L}} \underline{L}, A>+<\mathcal{D}_{A} \underline{L}, \underline{L}>\right)+\frac{1}{2} \underline{u}\left(<\mathcal{D}_{\underline{L}} L, A>+<\mathcal{D}_{A} L, \underline{L}>\right)+ \\
& +\frac{1}{2} \underline{L}(u)<\underline{L}, A>+\frac{1}{2} A(u)<\underline{L}, \underline{L}>+\frac{1}{2} \underline{L}(\underline{u})<L, A>+\frac{1}{2} A(\underline{u})<L, \underline{L}>= \\
& =u \underline{\xi}+\underline{u}\left(\eta+\kappa_{A N}\right) \\
& S_{\pi_{L}}=\frac{1}{2} u\left(<\mathcal{D}_{L} \underline{L}, \underline{L}>+<\mathcal{D}_{\underline{L}} \underline{L}, L>\right)+\frac{1}{2} \underline{u}\left(<\mathcal{D}_{L} L, \underline{L}>+<\underline{1} \mathcal{D}_{\underline{L}} L, L>\right)+ \\
& +\frac{1}{2} L(u)<\underline{L}, \underline{L}>+\frac{1}{2} \underline{L}(u)<\underline{L}, L>+\frac{1}{2} L(\underline{u})<L, \underline{L}>+\frac{1}{2} \underline{L}(\underline{u})<L, L>= \\
& =u \kappa_{N N}+\underline{u} \kappa_{N N}-2 b^{-1}-2 \\
& \quad=2
\end{aligned}
$$

As in the case of $\Omega$, we will use the energy estimate (125) for the equation (157). This implies:

$$
\begin{aligned}
\|\partial S \phi\|_{L^{2}\left(\Sigma_{t}\right)} & \lesssim\|\partial S \phi\|_{L^{2}\left(\Sigma_{t_{0}}\right)}+ \\
& +\int_{t_{0}}^{t}\left\|^{S} \hat{\pi}^{\alpha \beta} \cdot \mathcal{D}_{\alpha} \mathcal{D}_{\beta} \phi+\mathcal{D}^{\alpha{ }^{S}} \hat{\pi}_{\alpha \lambda} \cdot \mathcal{D}^{\lambda} \phi-\frac{1}{6} \mathcal{D}^{\lambda} t r^{S} \pi \cdot \mathcal{D}_{\lambda} \phi\right\|_{L^{2}\left(\Sigma_{\tau}\right)} d \tau
\end{aligned}
$$

Using $\hat{\pi}$ instead of $\pi$ will affect only two terms:

$$
\begin{aligned}
& { }^{S} \hat{\pi}_{L \underline{L}}={ }^{S} \pi_{L \underline{L}}-2 g_{L \underline{L}}=2 t \kappa_{N N}+2\left(1-b^{-1}\right) \\
& { }^{S} \hat{\pi}_{A A}={ }^{S} \pi_{A A}-2 g_{A A}=u\left(\underline{\chi}+\frac{1}{s}\right)+\underline{u}\left(\chi-\frac{1}{s}\right)
\end{aligned}
$$


As before, we will first deal with the terms which do not involve derivatives of the deformation tensor:

$$
\begin{aligned}
& \int_{t_{0}}^{t}\left\|{ }^{S} \hat{\pi}_{L L} \mathcal{D}_{\underline{L}} \mathcal{D}_{\underline{L}} \phi\right\|_{L^{2}\left(\Sigma_{\tau}\right)} d \tau \lesssim \int_{t_{0}}^{t}\left\|u \kappa_{N N}\left(\underline{L}(\underline{L} \phi)-2 \underline{\xi} A \phi+\kappa_{N N} \underline{L} \phi\right)\right\|_{L^{2}\left(\Sigma_{\tau}\right)} d \tau \\
& \lesssim \int_{t_{0}}^{t} \lambda^{-\bar{a}}\|u \underline{L}(\partial \phi)\|_{L^{2}\left(\Sigma_{\tau}\right)}+\tau \lambda^{-\bar{a}}\left(\tau^{\frac{1}{2}} \lambda^{-\bar{a}-\frac{1-a}{2}+\epsilon}+\lambda^{-\bar{a}}\right)\|\partial \phi\|_{L^{2}\left(\Sigma_{\tau}\right)} d \tau \\
& \lesssim \lambda^{-\epsilon} \sup _{\tau \in\left[t_{0}, t_{*}\right]} E^{\frac{1}{2}}(\partial \phi)(\tau) \\
& \int_{t_{0}}^{t}\left\|{ }^{S} \hat{\pi}_{\underline{L L}} \mathcal{D}_{L} \mathcal{D}_{L} \phi\right\|_{L^{2}\left(\Sigma_{\tau}\right)} d \tau \lesssim \int_{t_{0}}^{t}\left\|\left(4\left(b^{-1}-1\right)-2 \underline{u} \kappa_{N N}\right)\left(L(L \phi)+\kappa_{N N} L \phi\right)\right\|_{L^{2}\left(\Sigma_{\tau}\right)} d \tau \\
& \lesssim \int_{t_{0}}^{t} \lambda^{-\bar{a}}\|\tau L(\partial \phi)\|_{L^{2}\left(\Sigma_{\tau}\right)}+\tau \lambda^{-2 \bar{a}}\|\partial \phi\|_{L^{2}\left(\Sigma_{\tau}\right)} d \tau \lesssim \\
& \lesssim \lambda^{-\epsilon} \sup _{\tau \in\left[t_{0}, t_{*}\right]} E^{\frac{1}{2}}(\partial \phi)(\tau) \\
& \int_{t_{0}}^{t}\left\|{ }^{S} \hat{\pi}_{L A} \mathcal{D}_{A} \mathcal{D}_{\underline{L}} \phi\right\|_{L^{2}\left(\Sigma_{\tau}\right)} d \tau \lesssim \int_{t_{0}}^{t}\left\|2 u \kappa_{A N}(\underline{L}(A \phi)-\underline{\xi} L \phi-\eta \underline{L} \phi)\right\|_{L^{2}\left(\Sigma_{\tau}\right)} d \tau \\
& \lesssim \int_{t_{0}}^{t} \lambda^{-\bar{a}}\|u \underline{L}(\partial \phi)\|_{L^{2}\left(\Sigma_{\tau}\right)}+\tau \lambda^{-\bar{a}}\left(\tau^{\frac{1}{2}} \lambda^{-\bar{a}-\frac{1-a}{2}+\epsilon}+\lambda^{-\bar{a}}\right)\|\partial \phi\|_{L^{2}\left(\Sigma_{\tau}\right)} d \tau \\
& \lesssim \lambda^{-\epsilon} \sup _{\tau \in\left[t_{0}, t_{*}\right]} E^{\frac{1}{2}}(\partial \phi)(\tau) \\
& \int_{t_{0}}^{t}\left\|{ }^{S} \hat{\pi}_{\underline{L} A} \mathcal{D}_{L} \mathcal{D}_{A} \phi\right\|_{L^{2}\left(\Sigma_{\tau}\right)} d \tau \lesssim \int_{t_{0}}^{t}\left\|\left(u \underline{\xi}+\underline{u}\left(\eta+\kappa_{A N}\right)\right)\left(L(A \phi)+\kappa_{A N} L \phi\right)\right\|_{L^{2}\left(\Sigma_{\tau}\right)} d \tau \\
& \lesssim \int_{t_{0}}^{t}\left(\tau^{\frac{1}{2}} \lambda^{-\bar{a}-\frac{1-a}{2}+\epsilon}+\lambda^{-\bar{a}}\right)\|\tau L(\partial \phi)\|_{L^{2}\left(\Sigma_{\tau}\right)}+\tau \lambda^{-\bar{a}}\left(\tau^{\frac{1}{2}} \lambda^{-\bar{a}-\frac{1-a}{2}+\epsilon}+\lambda^{-\bar{a}}\right)\|\partial \phi\|_{L^{2}\left(\Sigma_{\tau}\right)} d \tau \\
& \lesssim \lambda^{-\epsilon} \sup _{\tau \in\left[t_{0}, t_{*}\right]} E^{\frac{1}{2}}(\partial \phi)(\tau) \\
& \int_{t_{0}}^{t}\left\|{ }^{S} \hat{\pi}_{L \underline{L}} \mathcal{D}_{L} \mathcal{D}_{\underline{L}} \phi\right\|_{L^{2}\left(\Sigma_{\tau}\right)} d \tau \lesssim \int_{t_{0}}^{t} \|\left(2 t \kappa_{N N}+2\left(1-b^{-1}\right)\right)\left(L(\underline{L} \phi)-\kappa_{N N} \underline{L} \phi+\right. \\
& \left.+2 \kappa_{A N} A \phi\right)\left\|_{L^{2}\left(\Sigma_{\tau}\right)} d \tau \lesssim \int_{t_{0}}^{t} \lambda^{-\bar{a}}\right\| \tau L(\partial \phi)\left\|_{L^{2}\left(\Sigma_{\tau}\right)}+\tau \lambda^{-2 \bar{a}}\right\| \partial \phi \|_{L^{2}\left(\Sigma_{\tau}\right)} d \tau \\
& \lesssim \lambda^{-\epsilon} \sup _{\tau \in\left[t_{0}, t_{*}\right]} E^{\frac{1}{2}}(\partial \phi)(\tau) \\
& \int_{t_{0}}^{t}\left\|{ }^{S} \hat{\pi}_{A A} \mathcal{D}_{A} \mathcal{D}_{A} \phi\right\|_{L^{2}\left(\Sigma_{\tau}\right)} d \tau \lesssim \int_{t_{0}}^{t} \|\left(2 s\left(\chi-\frac{1}{s}\right)-u \kappa_{A A}\right)(A(A \phi)-\chi \underline{L} \phi- \\
& -\operatorname{tr} \underline{\chi} L \phi)\left\|_{L^{2}\left(\Sigma_{\tau}\right)} d \tau \lesssim \int_{t_{0}}^{t} \lambda^{-\bar{a}}\right\| \tau A(\partial \phi)\left\|_{L^{2}\left(\Sigma_{\tau}\right)}+\tau \lambda^{-2 \bar{a}}\right\| \partial \phi \|_{L^{2}\left(\Sigma_{\tau}\right)} d \tau+ \\
& +\int_{t_{0}}^{t}\left\|\frac{1}{s} \kappa_{A A} u \partial \phi\right\|_{L^{2}\left(\Sigma_{\tau}\right)} d \tau \lesssim \lambda^{-\epsilon} \sup _{\tau \in\left[t_{0}, t_{*}\right]} E^{\frac{1}{2}}(\partial \phi)(\tau)+\int_{t_{0}}^{t}\left\|\frac{1}{s} \kappa_{A A} u \partial \phi(1-\bar{\eta})\right\|_{L^{2}\left(\Sigma_{\tau}\right)} d \tau
\end{aligned}
$$

To conclude the analysis of these terms, we have to deal with the last integral. 


$$
\begin{aligned}
& \int_{t_{0}}^{t}\left\|\frac{1}{s} \kappa_{A A} u \partial \phi(1-\bar{\eta})\right\|_{L^{2}\left(\Sigma_{\tau}\right)} d \tau \lesssim \int_{t_{0}}^{t} \frac{1}{\tau}\left\|\frac{1}{s} \kappa_{A A} u(1-\bar{\eta})\right\|_{L^{2}\left(\Sigma_{\tau}\right)} E^{\frac{1}{2}}(\partial \phi)(\tau) d \tau \lesssim \\
& \lesssim \int_{t_{0}}^{t}\left\|\frac{1}{s} \kappa_{A A}(1-\bar{\eta})\right\|_{L^{2}\left(\Sigma_{\tau}\right)} d \tau \sup _{\tau \in\left[t_{0}, t_{*}\right]} E^{\frac{1}{2}}(\partial \phi)(\tau)
\end{aligned}
$$

Due to the fact that

$$
\sup _{S_{t, u}}|\kappa| \lesssim \min \left\{s \lambda^{-\bar{a}-(1-a)}, \lambda^{-\bar{a}}\right\}
$$

the last $L^{2}$-norm can be estimated as follows :

$$
\begin{aligned}
& \left\|\frac{1}{s} \kappa_{A A}(1-\bar{\eta})\right\|_{L^{2}\left(\Sigma_{\tau}\right)}^{2} \lesssim \int_{0}^{1} \frac{1}{s^{2}} \int_{S_{\tau, u}}|\kappa|^{2} d \sigma d s+\int_{1}^{\frac{\tau}{2}} \frac{1}{s^{2}} \int_{S_{\tau, u}}|\kappa|^{2} d \sigma d s \lesssim \\
& \lesssim \int_{0}^{1} \frac{1}{s^{2}} s s^{2} \lambda^{-2 \bar{a}-2(1-a)} d s+\int_{1}^{\frac{\tau}{2}} \frac{1}{s^{2}} s \lambda^{-2 \bar{a}} d s \lesssim \lambda^{-2 \bar{a}-2(1-a)}+\lambda^{-2 \bar{a}} \ln \tau
\end{aligned}
$$

This result obviously implies:

$$
\int_{t_{0}}^{t}\left\|\frac{1}{s} \kappa_{A A}(1-\bar{\eta})\right\|_{L^{2}\left(\Sigma_{\tau}\right)} d \tau \lesssim \lambda^{-\epsilon}
$$

Next we investigate the terms which involve derivatives of the $t r^{S} \pi$. Before we start, let us first write $t r^{S} \pi$ in the form

$$
t r^{S} \pi=4+2 b^{-1}+2 s\left(\chi-\frac{1}{s}\right)-2 u \kappa_{A A}-2 t \kappa_{N N}
$$

The expression to evaluate is $\int_{t_{0}}^{t}\left\|\mathcal{D}^{\alpha}\left(t r^{S} \pi\right) \mathcal{D}_{\alpha} \phi\right\|_{L^{2}\left(\Sigma_{\tau}\right)} d \tau$. All the terms that appear from the differentiation of $t r^{S} \pi$, with the exception of $u \not \nabla\left(\kappa_{A A}\right)$ and $t \not\left(\kappa_{N N}\right)$, can be estimated in a similar manner like the " $Y$ "-term from the previous section. All these estimates yield the desired upper bound $\lambda^{-\epsilon} \sup _{\tau \in\left[t_{0}, t_{*}\right]} E^{\frac{1}{2}}(\partial \phi)(\tau)$. We show the proof for the term involving $\kappa_{A A}$. As before, we split the argument in the interior and the exterior part and use the corresponding integral estimates:

$$
\begin{aligned}
& \int_{t_{0}}^{t}\left\|u \not \nabla\left(\kappa_{A A}\right) \partial \phi\right\|_{L^{2}\left(\Sigma_{\tau}\right)} d \tau \lesssim \int_{t_{0}}^{t} \tau^{\frac{1}{2}} \sup _{0 \leq u \leq \frac{\tau}{2}}\left\|\not\left(\kappa_{A A}\right)\right\|_{L^{2}\left(S_{\tau, u}\right)} E^{\frac{1}{2}}(\partial \phi)(\tau) d \tau+ \\
& +\int_{t_{0}}^{t}\left\|\not\left(\kappa_{A A}\right)(1-\omega)\right\|_{L^{2}\left(\Sigma_{\tau}\right)} E^{\frac{1}{2}}(\partial \phi)(\tau) d \tau \lesssim\left(\lambda^{-\epsilon}+\int_{t_{0}}^{t}\left\|\not \nabla\left(\kappa_{A A}\right)(1-\omega)\right\|_{L^{2}\left(\Sigma_{\tau}\right)} d \tau\right) . \\
& \cdot \sup _{\tau \in\left[t_{0}, t_{*}\right]} E^{\frac{1}{2}}(\partial \phi)(\tau)
\end{aligned}
$$

We are left to investigate the norm under the last integral, for which we need the following weak estimate:

$$
\sup _{S_{\tau, u}}\left|\not \nabla\left(\kappa_{A A}\right)\right| \lesssim s \lambda^{-\bar{a}-2(1-a)}
$$


This is obtained immediately from the trivial bound

$$
\left|\frac{d}{d s}\left(\not \nabla\left(\kappa_{A A}\right)\right)\right| \lesssim\left|\partial^{3} g\right|+|\partial g| \cdot\left|\partial^{2} g\right|
$$

and the following estimate (30) satisfied by the metric $g$ :

$$
\left\|\partial^{1+m} g_{\lambda}\right\|_{L_{t}^{\infty} L_{x}^{\infty}} \lesssim \lambda^{-\bar{a}-(1-a) m},
$$

true for all integers $0 \leq m$. This implies then:

$$
\begin{aligned}
\left\|\not \nabla\left(\kappa_{A A}\right)(1-\omega)\right\|_{L^{2}\left(\Sigma_{\tau}\right)}^{2} & \lesssim \int_{0}^{1} \int_{S_{\tau, u}}\left|\not \nabla\left(\kappa_{A A}\right)\right|^{2} d \sigma d s+\int_{1}^{\frac{\tau}{2}} \int_{S_{\tau, u}}\left|\not \nabla\left(\kappa_{A A}\right)\right|^{2} d \sigma d s \lesssim \\
& \lesssim \int_{0}^{1} s s^{2} \lambda^{-2 \bar{a}-4(1-a)} d s+\int_{1}^{\frac{\tau}{2}}\left(\lambda^{-2 \bar{a}-(1-a)}+s^{-1} \lambda^{-2 \bar{a}}\right) d s \lesssim \\
& \lesssim \lambda^{-2 \bar{a}-4(1-a)}+\tau \lambda^{-2 \bar{a}-(1-a)}+\lambda^{-2 \bar{a}} \ln \tau
\end{aligned}
$$

This estimate implies then

$$
\int_{t_{0}}^{t}\left\|\not\left(\kappa_{A A}\right)(1-\omega)\right\|_{L^{2}\left(\Sigma_{\tau}\right)} d \tau \lesssim \lambda^{-\epsilon}
$$

which is, of course, the desired bound. In order to conclude our argument we have to estimate the term

$$
\int_{t_{0}}^{t}\left\|\mathcal{D}^{\alpha S} \hat{\pi}_{\alpha \beta} \cdot \mathcal{D}^{\beta} \phi\right\|_{L^{2}\left(\Sigma_{\tau}\right)} d \tau
$$

As in the discussion for the angular momentum vectorfield $\Omega$, in the decomposition of the expression above we encounter two type of terms:

- terms which contain derivatives of the deformation tensor, which we denote generically by $Y^{\prime}$

- terms which do not contain derivatives of the deformation tensor, which we denote generically by $X^{\prime}$. Through a very easy inspection, we have the estimates:

$$
\begin{aligned}
& \left|Y^{\prime}\right| \lesssim|Y|+|\not \nabla(\kappa)| \\
& \left|X^{\prime}\right| \lesssim|X|+\left|\frac{1}{s} \tau \kappa\right|
\end{aligned}
$$

As it can obviously be seen, these terms were covered previously, so this concludes our discussion concerning the scaling vectorfield $S$.

Remark Comparing with the investigation of $\Omega$, the discussion regarding $S$ had difficulties in evaluating the interior part of the integral terms, namely singularities appeared due to negative powers of $s$. This is determined by the fact that, for the interior region, in the case of $S, u$ appears as minimal weight, while for the discussion regarding $\Omega$, due also to $\Omega=s \cdot A, s$ is the weight that appeared more often. 


\section{The conclusion of the argument}

At the end of section 5, we reduced the local well-posedness result up to the proof of Theorem 5.1 , which we recall here in the form:

Theorem 9.1 Under the assumptions of Theorem 5.1 :

$$
\sup _{\tau \in\left[t_{0}, t_{*}\right]} E(\partial \phi)(\tau) \lesssim E(\partial \phi)\left(t_{0}\right)
$$

With the estimates obtained in the course of the previous two chapters, we can prove now this theorem. We will proceed as follows:

\subsection{Proof of the estimate (170) in the exterior region $\left\{s \gtrsim \frac{t}{2}\right\}$}

First we prove the following

Lemma 9.2 Under the assumptions of Theorem 5.1 we have the following commutator estimates:

$$
\begin{aligned}
\|[\partial, \Omega] \phi\|_{L^{2}\left(\Sigma_{t}\right)} & \lesssim \lambda^{-\epsilon}\|\partial \phi\|_{L^{2}\left(\Sigma_{t}\right)} \\
\|[\partial, S] \phi\|_{L^{2}\left(\Sigma_{t}\right)} & \lesssim\|\partial \phi\|_{L^{2}\left(\Sigma_{t}\right)}
\end{aligned}
$$

Proof It is obvious that the lemma will follow if we verify the commutator estimates for $\partial$ replaced with either $L, \underline{L}$ or $A$. For the angular momentum vectorfield $\Omega$ we have

$$
\begin{aligned}
& {[L, \Omega]=L(s \cdot A)-s \cdot A L=(1-s \chi) A,} \\
& {[\underline{L}, \Omega]=\underline{L}(s \cdot A)-s \cdot A \underline{L}=\left(1-2 b^{-1}-s \underline{\chi}\right) A+2 s\left(\kappa_{A N}-\eta\right) N,} \\
& {[A, \Omega]=A(s \cdot A)-s \cdot A^{2}=0,}
\end{aligned}
$$

while for the scaling vectorfield $S$, the calculations go like this:

$$
\begin{aligned}
& {[L, S]=\frac{1}{2} L(u) \underline{L}+\frac{1}{2} u[L, \underline{L}]+\frac{1}{2} L(\underline{u}) L+\frac{1}{2} \underline{u}[L, L]=\left(-u\left(\kappa_{A N}+\eta\right)\right) A+} \\
& +\left(1-\frac{1}{2} u \kappa_{N N}\right) L+\frac{1}{2} u \kappa_{N N} \underline{L} \\
& {[\underline{L}, S]=\frac{1}{2} \underline{L}(u) \underline{L}+\frac{1}{2} u[\underline{L}, \underline{L}]+\frac{1}{2} \underline{L}(\underline{u}) L+\frac{1}{2} \underline{u}[\underline{L}, L]=\left(-\underline{u}\left(\kappa_{A N}+\eta\right)\right) A+} \\
& +\left(1-b^{-1}+\frac{1}{2} \underline{u} \kappa_{N N}\right) L+\left(b^{-1}-\frac{1}{2} \underline{u} \kappa_{N N}\right) \underline{L} \\
& {[A, S]=\frac{1}{2} A(u) \underline{L}+\frac{1}{2} u[A, \underline{L}]+\frac{1}{2} A(\underline{u}) L+\frac{1}{2} \underline{u}[A, L]=\left(\frac{1}{2} u \operatorname{tr} \underline{\chi}+\frac{1}{2} \underline{u} \chi\right) A+} \\
& +u\left(\eta-\kappa_{A N}\right) T
\end{aligned}
$$

These equations together with the asymptotic estimates yield the desired outcome. 
Lemma 9.3 Under the assumptions of Theorem 5.1, the following estimates hold:

$$
\begin{aligned}
&\|\underline{u} \cdot A \partial \phi \cdot \bar{\eta}\|_{L^{2}\left(\Sigma_{t}\right)} \lesssim\|\Omega \partial \phi\|_{L^{2}\left(\Sigma_{t}\right)} \\
&\|\underline{u} \cdot L \partial \phi \cdot \bar{\eta}\|_{L^{2}\left(\Sigma_{t}\right)} \lesssim\|\Omega \partial \phi\|_{L^{2}\left(\Sigma_{t}\right)}+\|S \partial \phi\|_{L^{2}\left(\Sigma_{t}\right)}+\|\partial \phi\|_{L^{2}\left(\Sigma_{t}\right)} \\
&\|u \cdot \underline{L} \partial \phi \cdot \bar{\eta}\|_{L^{2}\left(\Sigma_{t}\right)} \lesssim\|\Omega \partial \phi\|_{L^{2}\left(\Sigma_{t}\right)}+\|S \partial \phi\|_{L^{2}\left(\Sigma_{t}\right)}+\|\partial \phi\|_{L^{2}\left(\Sigma_{t}\right)}
\end{aligned}
$$

Proof The first estimate is almost trivial due to the fact that in the exterior region $\underline{u} \approx$ $t \approx s$. We can conclude

$$
\|\underline{u} \cdot A \partial \phi \cdot \bar{\eta}\|_{L^{2}\left(\Sigma_{t}\right)} \lesssim\|s \cdot A \partial \phi\|_{L^{2}\left(\Sigma_{t}\right)}=\|\Omega \partial \phi\|_{L^{2}\left(\Sigma_{t}\right)}
$$

Let us observe that it is enough to prove only the estimates for $L$, the ones for $\underline{L}$ following immediately from

$$
u \underline{L}=2 S-\underline{u} L
$$

In order to prove the estimates for $L$ we use the equation:

$$
\begin{aligned}
\square_{g} \phi & =-\mathcal{D}_{L} \mathcal{D}_{\underline{L}} \phi+\mathcal{D}_{A} \mathcal{D}_{A} \phi= \\
& =-L(\underline{L} \phi)+A(A \phi)-\left(\kappa_{N N}+\frac{1}{2} \underline{\chi}\right) L \phi-\frac{1}{2} \chi \underline{L} \phi=0
\end{aligned}
$$

This yields of course

$$
|s \cdot L(\underline{L} \phi)| \lesssim|s \cdot A(A \phi)|+|L \phi|+|\underline{L} \phi|
$$

which in turn implies:

$$
\|\underline{u} \cdot L(\underline{L} \phi) \cdot \bar{\eta}\|_{L^{2}\left(\Sigma_{t}\right)} \lesssim\|s \cdot L(\underline{L} \phi)\|_{L^{2}\left(\Sigma_{t}\right)} \lesssim\|\Omega \partial \phi\|_{L^{2}\left(\Sigma_{t}\right)}+\|\partial \phi\|_{L^{2}\left(\Sigma_{t}\right)}
$$

Next we investigate $|\underline{u} \cdot L(L \phi)|$. We proceed like this:

$$
\begin{aligned}
|\underline{u} \cdot L(L \phi)| & =|2 S(L \phi)-u \cdot \underline{L}(L \phi)| \lesssim|S(L \phi)|+|u \cdot L(\underline{L} \phi)|+|u \cdot[L, \underline{L}] \phi| \lesssim \\
& \lesssim|S(L \phi)|+|u \cdot L(\underline{L} \phi)|+\left|u\left(\eta+\kappa_{A N}\right) A \phi\right|+\left|u \kappa_{N N} N \phi\right|
\end{aligned}
$$

This estimate together with the previous one yield:

$$
\|\underline{u} \cdot L(L \phi) \cdot \bar{\eta}\|_{L^{2}\left(\Sigma_{t}\right)} \lesssim\|\Omega \partial \phi\|_{L^{2}\left(\Sigma_{t}\right)}+\|S \partial \phi\|_{L^{2}\left(\Sigma_{t}\right)}+\|\partial \phi\|_{L^{2}\left(\Sigma_{t}\right)}
$$

Finally:

$$
|s \cdot L(A \phi)| \lesssim|s \cdot A(L \phi)|+|s \cdot[L, A] \phi| \lesssim|\Omega(L \phi)|+|s \cdot \chi A \phi|
$$

which enables us to infer that

$$
\|\underline{u} \cdot L(A \phi) \cdot \bar{\eta}\|_{L^{2}\left(\Sigma_{t}\right)} \lesssim\|s \cdot L(A \phi)\|_{L^{2}\left(\Sigma_{t}\right)} \lesssim\|\Omega \partial \phi\|_{L^{2}\left(\Sigma_{t}\right)}+\|\partial \phi\|_{L^{2}\left(\Sigma_{t}\right)}
$$

Putting altogether the previous results, we finally conclude:

$$
\begin{aligned}
E_{e x t}^{\frac{1}{2}}(\partial \phi)(t) & \lesssim\|\underline{u} \cdot A \partial \phi \cdot \bar{\eta}\|_{L^{2}\left(\Sigma_{t}\right)}+\|\underline{u} \cdot L \partial \phi \cdot \bar{\eta}\|_{L^{2}\left(\Sigma_{t}\right)}+\|u \cdot \underline{L} \partial \phi \cdot \bar{\eta}\|_{L^{2}\left(\Sigma_{t}\right)}+ \\
& +\|\partial \phi\|_{L^{2}\left(\Sigma_{t}\right)} \lesssim\|\Omega \partial \phi\|_{L^{2}\left(\Sigma_{t}\right)}+\|S \partial \phi\|_{L^{2}\left(\Sigma_{t}\right)}+\|\partial \phi\|_{L^{2}\left(\Sigma_{t}\right)} \lesssim \\
& \lesssim E^{\frac{1}{2}}(\partial \phi)\left(t_{0}\right)+\lambda^{-\epsilon} \sup _{\tau \in\left[t_{0}, t_{*}\right]} E^{\frac{1}{2}}(\partial \phi)(\tau)
\end{aligned}
$$




\subsection{Proof of the estimate (170) in the interior region $\left\{s \lesssim \frac{t}{2}\right\}$}

Due to the result obtained in Theorem 7.1, for our purposes it will be enough to consider $E_{\text {int }}(\nabla \phi)$, where $\nabla$ designates spatial derivatives. We will prove the following result:

Theorem 9.4 Under the assumptions of Theorem 5.1, we have the following estimate:

$$
E_{\text {int }}(\nabla \phi)(t) \lesssim E_{\text {int }}\left(\partial_{t} \phi\right)(t)+E(\partial \phi)\left(t_{0}\right)
$$

Proof The approach is direct, using integration by parts. When there is no ambiguity we will write the terms symbolically.

$$
E_{\text {int }}(\nabla \phi)(t)=t^{2} \int_{\Sigma_{t}} g^{i j} \cdot \partial \partial_{i} \phi \cdot \partial \partial_{j} \phi \cdot(1-\omega)+\int_{\Sigma_{t}}|\nabla \phi|^{2} \cdot(1-\omega)
$$

Due to the energy estimate (132), the second term is bounded easily by:

$$
\int_{\Sigma_{t}}|\nabla \phi|^{2} \cdot(1-\omega) \lesssim\left\|\partial \phi\left(t_{0}\right)\right\|_{L^{2}\left(\Sigma_{t_{0}}\right)} \lesssim E(\partial \phi)\left(t_{0}\right)
$$

For the first integral we proceed as follows:

$$
\begin{aligned}
t^{2} \int_{\Sigma_{t}} g^{i j} \cdot \partial \partial_{i} \phi \cdot \partial \partial_{j} \phi \cdot(1-\omega) & =t^{2} \int_{\Sigma_{t}} g^{i j} \cdot \partial_{t} \partial_{i} \phi \cdot \partial_{t} \partial_{j} \phi \cdot(1-\omega)+ \\
& +t^{2} \int_{\Sigma_{t}} g^{i j} \cdot g^{k l} \partial_{k} \partial_{i} \phi \cdot \partial_{l} \partial_{j} \phi \cdot(1-\omega)
\end{aligned}
$$

Obviously, the first term has the upper bound:

$$
t^{2} \int_{\Sigma_{t}} g^{i j} \cdot \partial_{t} \partial_{i} \phi \cdot \partial_{t} \partial_{j} \phi \cdot(1-\omega) \lesssim E_{\text {int }}\left(\partial_{t} \phi\right)(t),
$$

while for the second one we will use, as mentioned above, integration by parts

$$
\begin{aligned}
& t^{2} \int_{\Sigma_{t}} g^{i j} \cdot g^{k l} \cdot \partial_{k} \partial_{i} \phi \cdot \partial_{l} \partial_{j} \phi \cdot(1-\omega)= \\
& \quad=-t^{2} \int_{\Sigma_{t}} g^{i j} \cdot g^{k l} \cdot \partial_{i} \phi \cdot \partial_{k} \partial_{l} \partial_{j} \phi \cdot(1-\omega)+t^{2} \int_{\Sigma_{t}} g \cdot \partial g \cdot \partial \phi \cdot \partial^{2} \phi \cdot(1-\omega)+ \\
& \quad+t^{2} \int_{\Sigma_{t}} g \cdot g \cdot \partial \phi \cdot \partial^{2} \phi \cdot \partial \omega=t^{2} \int_{\Sigma_{t}}|\Delta \phi|^{2} \cdot(1-\omega)+ \\
& \quad+t^{2} \int_{\Sigma_{t}} g \cdot \partial g \cdot \partial \phi \cdot \partial^{2} \phi \cdot(1-\omega)+t^{2} \int_{\Sigma_{t}} g \cdot g \cdot \partial \phi \cdot \partial^{2} \phi \cdot \partial \omega
\end{aligned}
$$

Using the equation satisfied by $\phi$, we can infer that:

$$
\Delta \phi=\partial_{t t} \phi+\partial g \cdot \partial \phi
$$


If we plug in this expression for $\Delta \phi$ in the above computation and take into account the estimates for $\|\partial g\|_{L_{x}^{\infty}}$, we obtain the following estimate:

$$
\begin{aligned}
& t^{2} \int_{\Sigma_{t}} g^{i j} \cdot g^{k l} \partial_{k} \partial_{i} \phi \cdot \partial_{l} \partial_{j} \phi \cdot(1-\omega) \lesssim t^{2} \int_{\Sigma_{t}}\left(\left|\partial_{t t} \phi\right|^{2}+|\partial g|^{2} \cdot|\partial \phi|^{2}+\right. \\
& \left.\quad+|g| \cdot|\partial g| \cdot|\partial \phi| \cdot\left|\partial^{2} \phi\right|+|g| \cdot|g| \cdot|\partial \phi| \cdot\left|\partial^{2} \phi\right| \cdot|\partial \omega|\right) \cdot(1-\omega) \lesssim \\
& \quad \lesssim E_{i n t}\left(\partial_{t} \phi\right)(t)+\lambda^{-\epsilon} E(\partial \phi)\left(t_{0}\right)+\lambda^{-\epsilon} E^{\frac{1}{2}}(\partial \phi)\left(t_{0}\right) \cdot E_{i n t}^{\frac{1}{2}}(\partial \phi)(t)+ \\
& \quad+E^{\frac{1}{2}}(\partial \phi)\left(t_{0}\right) \cdot E_{\text {int }}^{\frac{1}{2}}(\partial \phi)(t)
\end{aligned}
$$

Putting together (179),(180),(182), we conclude that

$$
E_{\text {int }}(\nabla \phi)(t) \lesssim E_{\text {int }}\left(\partial_{t} \phi\right)(t)+E(\partial \phi)\left(t_{0}\right)+E^{\frac{1}{2}}(\partial \phi)\left(t_{0}\right) \cdot E_{\text {int }}^{\frac{1}{2}}(\partial \phi)(t)
$$

which clearly implies the result claimed.

Remark The conclusion of this section, (170), will then be the cumulative result of (123),(177) and (178).

\section{References}

[1] Bahouri, H.; Chemin, J.-Y. Equations D'ondes Quasilineaires et Effet Dispersif. Internat. Math. Res. Notices 1999, 21, 1141-1178.

[2] Bahouri, H.; Chemin, J.-Y. Equations D'ondes Quasilineaires et Estimation de Strichartz. Amer. J. Math. 1999, $121(6)$, 1337-1377.

[3] Hughes, T.J.R.; Kato, T.; Marsden, J.E. Well-Posed Quasilinear Second-Order Hyperbolic Systems with Applications to Nonlinear Elastodynamics and General Relativity. Arch. Rational Mech. Anal. 1976, 63(3), 273-294.

[4] Kapitanskij, L.V. Estimates for Norms in Besov and Lizorkin-Triebel Spaces for Solutions of Second-Order Linear Hyperbolic Equations. J. Soviet Math. 1991, 56(2), 2348-2389.

[5] Klainerman, S. Uniform Decay Estimates and the Lorentz Invariance of the Classical Wave Equation. Comm. Pure Appl. Math. 1985, 38(3), 321-332.

[6] Klainerman, S. A Commuting Vectorfield Approach to Strichartz-Type Inequalities and Applications to Quasilinear Wave Equations. Internat. Math. Res. Notices 2001, 23(5), 221-274.

[7] Klainerman, S.; Rodnianski, I. Improved Local Well-Posedness for Quasilinear Wave Equations in Dimension Three. Preprint 
[8] Klainerman, S.; Rodnianski, I. Rough Solutions of the Einstein Vacuum Equations. C. R. Math. Acad. Sci. Paris 2002, $334(2), 125-130$.

[9] Lindblad, H. Counterexamples to Local Existence for Quasilinear Wave Equations. Math. Res. Letters 1998, 5(5), 605-622.

[10] Mockenhaupt, G.; Seeger, A.; Sogge, C.D. Local Smoothing of Fourier Integral Operators and Carleson-Sjolin Estimates. J. Amer. Math. Soc. 1993, 6(1), 65-130.

[11] Ponce, G.; Sideris, T. Local Regularity of Nonlinear Wave Equations in Three Space Dimensions. Comm. Partial Differential Equations 1993, 18(1-2), 169-177.

[12] Smith, H. A Parametrix Construction for Wave Equations with $C^{1,1}$ Coefficients. Ann. Inst. Fourier 1998, 48(3), 797-835.

[13] Smith, H.; Sogge, C.D. On Strichartz and Eigenfunction Estimates for Low Regularity Metrics. Math. Res. Letters 1994, 1(6), 729-737.

[14] Smith, H.; Tataru, D. Sharp Counterexamples for Strichartz Estimates for Low Regularity Metrics. Math. Res. Letters 2002, 9(2-3), 199-204.

[15] Smith, H.; Tataru, D. Sharp local well-posedness results for the nonlinear wave equation. Preprint

[16] Tataru, D. Strichartz Estimates for Operators with Nonsmooth Coefficients and the Nonlinear Wave Equation. Amer. J. Math. 2000, 122(2), 349-376.

[17] Tataru, D. Strichartz Estimates for Second Order Hyperbolic Operators with Nonsmooth Coefficients. II. Amer. J. Math. 2001, $123(3)$, 385-423. 